\title{
Asymmetric orbifolds and grand unification
}

\author{
Zurab Kakushadze* and S.-H. Henry Tye ${ }^{\dagger}$ \\ Newman Laboratory of Nuclear Studies, Cornell University, Ithaca, New York 14853-5001
}

(Received 16 July 1996)

\begin{abstract}
We generalize the rules for the free fermionic string construction to include other asymmetric orbifolds with world-sheet bosons. We use these rules to construct various grand unified string models that involve level-three current algebras. We present the explicit construction of three classes of three-chiral-family grand unified models in the heterotic string theory. Each model has five left-handed, and two right-handed families, and an adjoint Higgs representation. Two of them are $\mathrm{SO}(10)$ and the third is $\mathrm{E}_{6}$. With Wilson lines and/or varying moduli, we show how other three-family grand unified models, such as $\mathrm{SO}(10)$ and $\mathrm{SU}(5)$, may be constructed from them. [S0556-2821(96)01924-8]

PACS number(s): 11.25.Mj, 12.10.Dm
\end{abstract}

\section{INTRODUCTION}

The construction of string models has a long history. The number of consistent string models is clearly very large (one may consider various string models as different classical string vacua of a single theory; in this case, we are talking about the construction of classical vacua). The best understood string models are probably those obtained via toroidal compactification, along with their orbifolds [1]. However, the orbifold construction is still not fully explored. This is in part due to the lack of simple rules for constructing such models, in particular, asymmetric orbifolds [2].

The first class of asymmetric orbifold string models is composed of the free fermionic string models [3]. Although this class of models is rather restrictive (allowing only multiple $\mathbf{Z}_{2}$ twists), the rules for such model building are quite simple. As a result, rather complicated models can be readily constructed. For example, the first level-two grand unified models were constructed using these rules [4]. It is natural to attempt to generalize these rules to include more general orbifolds. It is not surprising that the rules become more involved as one tries to include more general types of orbifolds. At a certain point, the rules will become too complicated to be of any practical use. It seems that some intermediate ground is called for. In this paper, we present explicit rules for the construction of a rather large class of, but not all, asymmetric orbifold models. There are many interesting orbifold and free fermionic string constructions in the literature $[5,6]$. The rules should be useful for the bookkeeping of complicated model building, as is the case in the search for three-family grand unified string models.

The rules for the asymmetric orbifold construction attempt to combine the approach of Ref. [2] with those for the free fermionic string models [3]. Here we shall follow the notations of Ref. [7]. We shall impose the consistency requirements on the string one-loop partition function in the light-cone gauge: (i) one-loop modular invariance; (ii) world-sheet supersymmetry (if present), which ensures

\footnotetext{
*Present address: Lyman Laboratory, Harvard University, Cambridge, MA 02138. Electronic address: zurab@hepth.cornell.edu

${ }^{\dagger}$ Electronic address: tye@ hepth.cornell.edu
}

space-time Lorentz invariance in the covariant gauge; and (iii) the physically sensible projection; this means that the contribution of a space-time fermionic (bosonic) degree of freedom to the partition function counts as - (+) 1. In all cases that can be checked, this condition plus the one-loop modular invariance and factorization implies multiloop modular invariance.

The building blocks of the one-loop partition function of any specific string model are the (appropriate) characters of the world-sheet fermions and bosons. The fermion characters are the same as the ones used in the free fermionic string models. The characters for bosons are combined from two types, those for twisted chiral bosons and those for chiral boson lattices. A key to obtaining relatively simple rules is the choice of basis for the chiral lattices. They are chosen so that, up to phases, all the chiral lattice characters are permuted under any modular transformation, as is the case for the chiral fermion characters. Our discussion shall focus on heterotic strings compactified to four space-time dimensions.

As in the free fermionic string model constructions, the rules may be used to build new models without direct reference to their original partition functions and/or the characters. This turns out to be useful because the partition function can get rather complicated, while the rules are relatively easy to use. In this paper, we consider Abelian orbifolds. For a general lattice, the sublattice invariant under a twist may be difficult to identify. Sometimes, it is easier to start with a lattice whose invariant sublattice is obvious, and then introduce background fields, in particular Wilson lines, that commute with the twists. In fact, this approach is very useful in the symmetric orbifold construction [8]. Here, our work can be considered as a generalization of their work to the asymmetric orbifold case. If the Wilson lines do not commute with the twists, we may first construct the model with the Wilson lines, and then perform the twists on the resulting model, as we shall do in this paper. Alternatively, we may use the non-Abelian orbifold construction. The rules given here can be used as a basis for further generalization to the non-Abelian orbifold case, which will be discussed elsewhere [9].

After summarizing the rules, we use them to construct various grand unified models in the four-dimensional heterotic string theory. It is well known that, in field theory, an 
TABLE I. The massless spectra of $\mathrm{SO}(10)$ models from three different $\mathbf{Z}_{6}$ orbifolds: (1) and (2) $\mathrm{SU}(2)_{1} \otimes \mathrm{SU}(2)_{3} \otimes \mathrm{SO}(10)_{3} \otimes \mathrm{U}(1)^{3}$, and (3) $\mathrm{SU}(2)_{1} \otimes \mathrm{SO}(10)_{3} \otimes \mathrm{U}(1)^{4}$. The $\mathrm{U}(1)$ normalization radii are given at the bottom of the table. The graviton, dilaton, and gauge supermultiplets are not shown.

\begin{tabular}{|c|c|c|c|}
\hline$M$ & $\begin{array}{c}\mathrm{SU}(2)^{2} \otimes \mathrm{SO}(10) \otimes \mathrm{U}(1)^{3} \\
(\mathbf{1}, \mathbf{1}, \mathbf{4 5})(0,0,0) \\
(\mathbf{1}, \mathbf{3}, \mathbf{1})(0,0,0)\end{array}$ & $\begin{array}{c}\mathrm{SU}(2)^{2} \otimes \mathrm{SO}(10) \otimes \mathrm{U}(1)^{3} \\
(\mathbf{1}, \mathbf{1}, \mathbf{4 5})(0,0,0) \\
(\mathbf{1}, \mathbf{3}, \mathbf{1})(0,0,0)\end{array}$ & $\begin{array}{c}\mathrm{SU}(2) \otimes \mathrm{SO}(10) \otimes \mathrm{U}(1)^{4} \\
\quad(\mathbf{1 , 4 5})(0,0,0,0) \\
(\mathbf{1}, \mathbf{1})(0,0,0,0)_{L}\end{array}$ \\
\hline$U$ & $\begin{array}{c}(\mathbf{1}, \mathbf{1}, \mathbf{1})(0,-6,0)_{L} \\
2(\mathbf{1}, \mathbf{4}, \mathbf{1})(0,+3,0)_{L} \\
2(\mathbf{1}, \mathbf{2}, \mathbf{1})(0,-3,0)_{L}\end{array}$ & $\begin{array}{c}(\mathbf{1}, \mathbf{1}, \mathbf{1})(0,+6,0)_{L} \\
2(\mathbf{1}, \mathbf{1}, \mathbf{1})( \pm 3,-3,0)_{L} \\
2(\mathbf{2}, \mathbf{2}, \mathbf{1})(0,0,0)_{L}\end{array}$ & $\begin{array}{c}(\mathbf{1}, \mathbf{1})(0,+6,0,0)_{L} \\
2(\mathbf{1}, \mathbf{1})(0,-3, \pm 3,0)_{L}\end{array}$ \\
\hline & $\begin{array}{l}2(\mathbf{1}, \mathbf{2}, \mathbf{1 6})(0,-1,-1)_{L} \\
2(\mathbf{1}, \mathbf{2}, \mathbf{1 0})(0,-1,+2)_{L}\end{array}$ & $\begin{array}{l}2(\mathbf{1}, \mathbf{1}, \mathbf{1 6})( \pm 1,+1,-1)_{L} \\
2(\mathbf{1}, \mathbf{1}, \mathbf{1 0})( \pm 1,+1,+2)_{L}\end{array}$ & $\begin{array}{l}2(\mathbf{1}, \mathbf{1 6})(0,+1, \pm 1,-1)_{L} \\
2(\mathbf{1}, \mathbf{1 0})(0,+1, \pm 1,+2)_{L}\end{array}$ \\
\hline$T 3$ & $\begin{array}{c}2(\mathbf{1}, \mathbf{2}, \mathbf{1})(0,-1,-4)_{L} \\
(\mathbf{1}, \mathbf{1}, \mathbf{1 6})(0,+2,-1)_{L} \\
(\mathbf{1}, \mathbf{1}, \mathbf{1 0})(0,+2,+2)_{L} \\
(\mathbf{1}, \mathbf{1}, \mathbf{1})(0,+2,-4)_{L} \\
(\mathbf{1}, \overline{\mathbf{1}})( \pm 1,+1,+1)_{L}\end{array}$ & $\begin{array}{c}2(\mathbf{1}, \mathbf{1}, \mathbf{1})( \pm 1,+1,-4)_{L} \\
(\mathbf{1}, \mathbf{1}, \mathbf{1 6})(0,-2,-1)_{L} \\
(\mathbf{1}, \mathbf{1}, \mathbf{1 0})(0,-2,+2)_{L} \\
(\mathbf{1}, \mathbf{1}, \mathbf{1})(0,-2,-4)_{L} \\
(\mathbf{1}, \mathbf{2}, \overline{\mathbf{1 6}})(0,-1,+1)_{L}\end{array}$ & $\begin{array}{c}2(\mathbf{1}, \mathbf{1})(0,+1, \pm 1,-4)_{L} \\
(\mathbf{1}, \mathbf{1 6})(0,-2,0,-1)_{L} \\
(\mathbf{1}, \mathbf{1 0})(0,-2,0,+2)_{L} \\
(\mathbf{1}, \mathbf{1})(0,-2,0,-4)_{L} \\
(\mathbf{1}, \overline{\mathbf{1 6}})( \pm 1,-1,0,+1)_{L}\end{array}$ \\
\hline$T 6$ & $\begin{array}{c}(\mathbf{1}, \mathbf{1}, \mathbf{1 0})( \pm 1,+1,-2)_{L} \\
(\mathbf{1}, \mathbf{1}, \mathbf{1})( \pm 1,+1,+4)_{L}\end{array}$ & $\begin{array}{c}(\mathbf{1}, \mathbf{2}, \mathbf{1 0})(0,-1,-2)_{L} \\
(\mathbf{1}, \mathbf{2}, \mathbf{1})(0,-1,+4)_{L}\end{array}$ & $\begin{array}{c}(\mathbf{1}, \mathbf{1 0})( \pm 1,-1,0,-2)_{L} \\
(\mathbf{1}, \mathbf{1})( \pm 1,-1,0,+4)_{L}\end{array}$ \\
\hline$T 2$ & $\begin{array}{c}(\mathbf{2}, \mathbf{2}, \mathbf{1})(0,0,0)_{L} \\
(\mathbf{2}, \mathbf{4}, \mathbf{1})(0,0,0)_{L} \\
(\mathbf{1}, \mathbf{1}, \mathbf{1})( \pm 3,-3,0)_{L}\end{array}$ & $\begin{array}{c}(\mathbf{2}, \mathbf{1}, \mathbf{1})( \pm 3,0,0)_{L} \\
(\mathbf{1}, \mathbf{4}, \mathbf{1})(0,+3,0)_{L} \\
2(\mathbf{1}, \mathbf{2}, \mathbf{1})(0,-3,0)_{L} \\
(\mathbf{1}, \mathbf{2}, \mathbf{1})(0,+3,0)_{L}\end{array}$ & $\begin{array}{c}(\mathbf{2}, \mathbf{1})(0,0, \pm 3,0)_{L} \\
(\mathbf{1}, \mathbf{1})( \pm 3,+3,0,0)_{L}\end{array}$ \\
\hline $\mathrm{U}(1)$ & $(1 / \sqrt{6}, 1 / 3 \sqrt{2}, 1 / 6)$ & $(1 / \sqrt{6}, 1 / 3 \sqrt{2}, 1 / 6)$ & $(1 / \sqrt{6}, 1 / 3 \sqrt{2}, 1 / \sqrt{6}, 1 / 6)$ \\
\hline
\end{tabular}

adjoint Higgs (or other appropriate higher-dimensional) representation is necessary for a grand unified gauge group to break spontaneously to the $\mathrm{SU}(3) \otimes \mathrm{SU}(2) \otimes \mathrm{U}(1)$ gauge group of the standard model. It is also known that, for current algebras at level 1, space-time supersymmetry with chiral fermions does not coexist with massless scalar fields in the adjoint or higher-dimensional representations of the gauge group in heterotic string models. From these facts, one concludes that a grand unified model in the superstring theory is possible only if the grand unified gauge group comes from current algebras at levels higher than 1. Grand unified models with level-2 current algebras are not difficult to construct $[4,10]$. All models of this type have an even number of chiral families. There are many attempts to search for grand unification with three chiral families, so far unsuccessful [6]. Our own investigation is consistent with the observation that level- 2 models do not yield three chiral families.

The next obvious step is to go to higher levels. The central charge of higher-level current algebras increases quite rapidly. Intuitively, the feature of three chiral families seems to come most naturally with a $\mathbf{Z}_{3}$ orbifold. String consistency naturally leads one to level-3 current algebras. As a result of this consideration, a level-3 current algebra looks most promising. Our search indicates that a $\mathbf{Z}_{3}$ orbifold alone does not yield three families. However, a $\mathbf{Z}_{6}$ orbifold can yield three chiral families; more specifically, it yields five lefthanded and two right-handed families. In the construction of three-family grand unification, we shall impose an additional condition to help shrink the size of the moduli space of models: the presence of an asymptotically free hidden sector which can become strong above the electroweak scale. In this paper, we consider three different asymmetric $\mathbf{Z}_{6}$ orbi- folds. The largest grand unified gauge symmetries in these three orbifolds are $\mathrm{SO}(10), \mathrm{SO}(10)$, and $\mathrm{E}_{6}$, respectively. From these models, we may obtain other models by one of the three (not inequivalent) ways: (1) changing the moduli, (2) introducing or modifying the Wilson lines, and (3) giving some of the massless Higgs fields nonzero vacuum expectation values in the effective field theory. In particular, it is easy to convert the $\mathrm{E}_{6}$ model to another $\mathrm{SO}(10)$ model. The massless spectra of the three $\mathrm{SO}(10)$ models coming from the three different asymmetric $\mathbf{Z}_{6}$ orbifolds are given in Table I. There, each of the U(1) charges is normalized so that the lowest allowed values are \pm 1 , with conformal highest weight $r^{2} / 2$, where $r$ is the compactification radius of the corresponding chiral world-sheet boson. The states are grouped into sectors, the untwisted $U$ and the twisted $T$ sectors. This grouping follows from the string construction and implements the selection rules for string couplings.

Exploring the moduli space of the first $\mathrm{SO}(10)$ model, we find other closely related $\mathrm{SO}(10)$ models. Their massless spectra are given in Table II. The second and the third $\mathrm{SO}(10)$ models in Table I are somewhat isolated points in their respective moduli space, since changing the moduli typically damages the strong-interacting feature of the hidden sector. By turning on an appropriate Wilson line on any of the SO(10) models, an SU(5) model appears. The same Wilson line acting on the $\mathrm{E}_{6}$ model yields an SU(6) model. The massless spectra of the $\mathrm{E}_{6}$ model, the SU(6) model, and their closely related SU(5) model are given in Table III. The same procedure can be used to obtain other variations of the above models. For example, we may break the grand unified gauge symmetry to that of the standard model. We note that all the above models share the following features: (1) five left-handed and two right-handed chiral families of the grand 
TABLE II. The massless spectra of the three models connected by a continuous parameter: (1) $\mathrm{SU}(2)_{1} \otimes \mathrm{SO}(10)_{3} \otimes \mathrm{U}(1)^{4},(2) \mathrm{SU}(2)_{1}$ $\otimes \mathrm{SO}(10)_{3} \otimes \mathrm{U}(1)^{3}$, and (3) $\mathrm{SU}(2)_{1} \otimes \mathrm{SO}(10)_{3} \otimes \mathrm{U}(1)^{2}$. For these models the gauge group $R$ in the original moduli space is also shown. The $\mathrm{U}(1)$ normalization radii are given at the bottom of the table. The gravity, dilaton, and gauge supermultiplets are not shown.

\begin{tabular}{|c|c|c|c|}
\hline$M$ & $\mathrm{SU}(2) \otimes \mathrm{SO}(10) \otimes \mathrm{U}(1)^{4}$ & $\mathrm{SU}(2) 4 \otimes \mathrm{SO}(10) \otimes \mathrm{U}(1)^{3}$ & $\mathrm{SU}(2) \otimes \mathrm{SO}(10) \otimes \mathrm{U}(1)^{2}$ \\
\hline \multirow[t]{3}{*}{$R$} & $\mathrm{SU}(3) \otimes \mathrm{SU}(3)$ & $\mathrm{SU}(3) \otimes \mathrm{U}(1)^{2}$ & $\mathrm{U}(1)^{4}$ \\
\hline & $(\mathbf{1}, \mathbf{4 5})(0,0,0,0)$ & $(\mathbf{1}, \mathbf{4 5})(0,0,0)$ & $(\mathbf{1}, \mathbf{4 5})(0,0)$ \\
\hline & $2(\mathbf{1}, \mathbf{1})(0,+12,0,0)_{L}$ & $2(\mathbf{1}, \mathbf{1})(0,+12,0)_{L}$ & $4(\mathbf{1}, \mathbf{1})(0,0)$ \\
\hline \multirow[t]{7}{*}{$U$} & $2(\mathbf{1}, \mathbf{1})(0,0,+12,0)_{L}$ & $2(\mathbf{1}, \mathbf{1})(0,0,0)$ & \\
\hline & $3(\mathbf{1}, \mathbf{1})(0,-6,0,0)_{L}$ & $3(\mathbf{1}, \mathbf{1})(0,-6,0)_{L}$ & \\
\hline & $3(\mathbf{1}, \mathbf{1})(0,0,-6,0)_{L}$ & & \\
\hline & $2(\mathbf{1}, \mathbf{1 6})(0,+2,+2,-1)_{L}$ & $3(\mathbf{1}, \mathbf{1 6})(0,+2,-1)_{L}$ & $5(1,16)(0,-1)_{L}$ \\
\hline & $2(\mathbf{1}, \mathbf{1 0})(0,+2,+2,+2)_{L}$ & $3(\mathbf{1}, \mathbf{1 0})(0,+2,+2)_{L}$ & $5(\mathbf{1}, \mathbf{1 0})(0,+2)_{L}$ \\
\hline & $2(\mathbf{1}, \mathbf{1})(0,+2,+2,-4)_{L}$ & $3(\mathbf{1}, \mathbf{1})(0,+2,-4)_{L}$ & $5(\mathbf{1}, \mathbf{1})(0,-4)_{L}$ \\
\hline & $(\mathbf{1}, \mathbf{1 6})(0,-4,-4,-1)_{L}$ & $2(\mathbf{1}, \mathbf{1 6})(0,-4,-1)_{L}$ & \\
\hline \multirow[t]{9}{*}{$T 3$} & $(\mathbf{1}, \mathbf{1 0})(0,-4,-4,+2)_{L}$ & $2(\mathbf{1}, \mathbf{1 0})(0,-4,+2)_{L}$ & \\
\hline & $(\mathbf{1}, \mathbf{1})(0,-4,-4,-4)_{L}$ & $2(\mathbf{1}, \mathbf{1})(0,-4,-4)_{L}$ & \\
\hline & $(\mathbf{1}, \mathbf{1 6})(0,-4,+2,-1)_{L}$ & & \\
\hline & $(\mathbf{1}, \mathbf{1 0})(0,-4,+2,+2)_{L}$ & & \\
\hline & $(\mathbf{1}, \mathbf{1})(0,-4,+2,-4)_{L}$ & & \\
\hline & $(\mathbf{1}, \mathbf{1 6})(0,+2,-4,-1)_{L}$ & & \\
\hline & $(\mathbf{1}, \mathbf{1 0})(0,+2,-4,+2)_{L}$ & & \\
\hline & $(\mathbf{1}, \mathbf{1})(0,+2,-4,-4)_{L}$ & & \\
\hline & $(\mathbf{1}, \overline{\mathbf{1 6}})( \pm 1,+1,+1,+1)_{L}$ & $(\mathbf{1}, \overline{\mathbf{1 6}})( \pm 1,+1,+1)_{L}$ & $(\mathbf{1}, \overline{\mathbf{1 6}})( \pm 1,+1)_{L}$ \\
\hline \multirow[t]{3}{*}{$T 6$} & $(\mathbf{1}, \mathbf{1 0})( \pm 1,+1,+1,-2)_{L}$ & $(\mathbf{1}, \mathbf{1 0})( \pm 1,+1,-2)_{L}$ & $(\mathbf{1}, \mathbf{1 0})( \pm 1,-2)_{L}$ \\
\hline & $(\mathbf{1}, \mathbf{1})( \pm 1,+1,+1,+4)_{L}$ & $(\mathbf{1}, \mathbf{1})( \pm 1,+1,+4)_{L}$ & $(\mathbf{1}, \mathbf{1})( \pm 1,+4)_{L}$ \\
\hline & $2(\mathbf{2}, \mathbf{1})(0,-3,-3,0)_{L}$ & $2(\mathbf{2}, \mathbf{1})(0, \pm 3,0)_{L}$ & $6(\mathbf{2}, \mathbf{1})(0,0)_{L}$ \\
\hline \multirow[t]{3}{*}{$T 2$} & $(\mathbf{2}, \mathbf{1})(0, \pm 9,+3,0)_{L}$ & $(\mathbf{2}, \mathbf{1})(0, \pm 9,0)_{L}$ & \\
\hline & $(\mathbf{2}, \mathbf{1})(0,+3, \pm 9,0)_{L}$ & & \\
\hline & $(\mathbf{1}, \mathbf{1})( \pm 3,-3,-3,0)_{L}$ & $(\mathbf{1}, \mathbf{1})( \pm 3,-3,0)_{L}$ & $(\mathbf{1}, \mathbf{1})( \pm 3,0)_{L}$ \\
\hline $\mathrm{U}(1)$ & $(1 / \sqrt{6}, 1 / 6 \sqrt{2}, 1 / 6 \sqrt{2}, 1 / 6)$ & $(1 / \sqrt{6}, 1 / 6 \sqrt{2}, 1 / 6)$ & $(1 / \sqrt{6}, 1 / 6)$ \\
\hline
\end{tabular}

unified gauge symmetry, (2) one adjoint Higgs field, and (3) an asymptotically free $\mathrm{SU}(2)$ hidden sector, whose fine structure coupling at the string scale is 3 times that of the grand unified gauge group. Furthermore, many of them have a nontrivial (but not asymptotically free) gauge symmetry that may play the role of a horizontal symmetry, or as a messenger or intermediate sector. Although these models do not contain Higgs fields in higher representations (i.e., other than adjoint) of the grand unified gauge group, it is possible that higher-dimensional operators present in the string theory may play equivalent roles. Clearly, detailed analyses are necessary to see if this happens in any of the above models and if supersymmetry is dynamically broken.

It is clear that the three types of $\mathbf{Z}_{6}$ orbifolds considered in this paper are quite similar. Although these three are probably not exhaustive, a distinct feature of grand unification in string theory is the very limited number of possibilities, as compared to standard model building. At the same time, the grand unified models presented here are quite complex. This complicated structure hopefully bodes well for phenomenology.

The construction of the first supersymmetric $\mathrm{SO}(10)$ grand unified model with five left-handed and two righthanded families was presented in Ref. [11]. This model has gauge symmetry $\mathrm{SU}(2)_{1} \otimes \mathrm{SU}(2)_{3} \otimes \mathrm{SO}(10)_{3} \otimes \mathrm{U}(1)^{3}$. Here, we give the details of that construction, using the rules developed in this paper. Although the derivation of the rules is somewhat complicated, the rules themselves, summarized at the end of Sec. III, are relatively easy to use. So the reader may skip the derivation at first reading. To get a better feeling of the model as well as the rules, we present an alternative way to construct this model.

In Sec. II, we briefly review the fermion and the boson characters and other background material that we shall use later. In Sec. III, we derive the rules for model building. To be specific, we consider only heterotic strings compactified to four spacetime dimensions. The rules for model building are summarized. For most of the applications in this paper, a simplified version of the rules is used. The simplified rules are also summarized at the end of Sec. III. In Sec. IV, we use these rules to construct the three-family $\mathrm{SO}(10)$ grand unified string model given in Ref. [11]. The massless spectrum of this model is given in the first column in Table I. In Sec. $\mathrm{V}$, we discuss an alternative construction of the same model. The quantum numbers of the particles are easier to identify in this construction. Section VI contains a discussion of the moduli space of the above construction, in which the above model is a special point in the moduli space. Another special point yields a model with gauge symmetry $\mathrm{SU}(2)_{1}$ $\otimes \mathrm{SO}(10)_{3} \otimes \mathrm{U}(1)^{4}$. Generic points in this moduli space have 
TABLE III. The massless spectra of the $\mathrm{E}_{6}, \mathrm{SU}(6)$, and $\mathrm{SU}(5)$ models from the same $\mathbf{Z}_{6}$ orbifold: (1) $\mathrm{SU}(2)_{1} \otimes\left(\mathrm{E}_{6}\right)_{3} \otimes \mathrm{U}(1)^{3}$, (2) $\mathrm{SU}(2)_{1} \otimes \mathrm{SU}(2)_{3} \otimes \mathrm{SU}(6)_{3} \otimes \mathrm{U}(1)^{3}$, and (3) $\mathrm{SU}(2)_{1} \otimes \mathrm{SU}(5)_{3} \otimes \mathrm{U}(1)^{5}$. The U(1) normalization radii are given at the bottom of the table. The gravity, dilaton, and gauge supermultiplets are not shown.

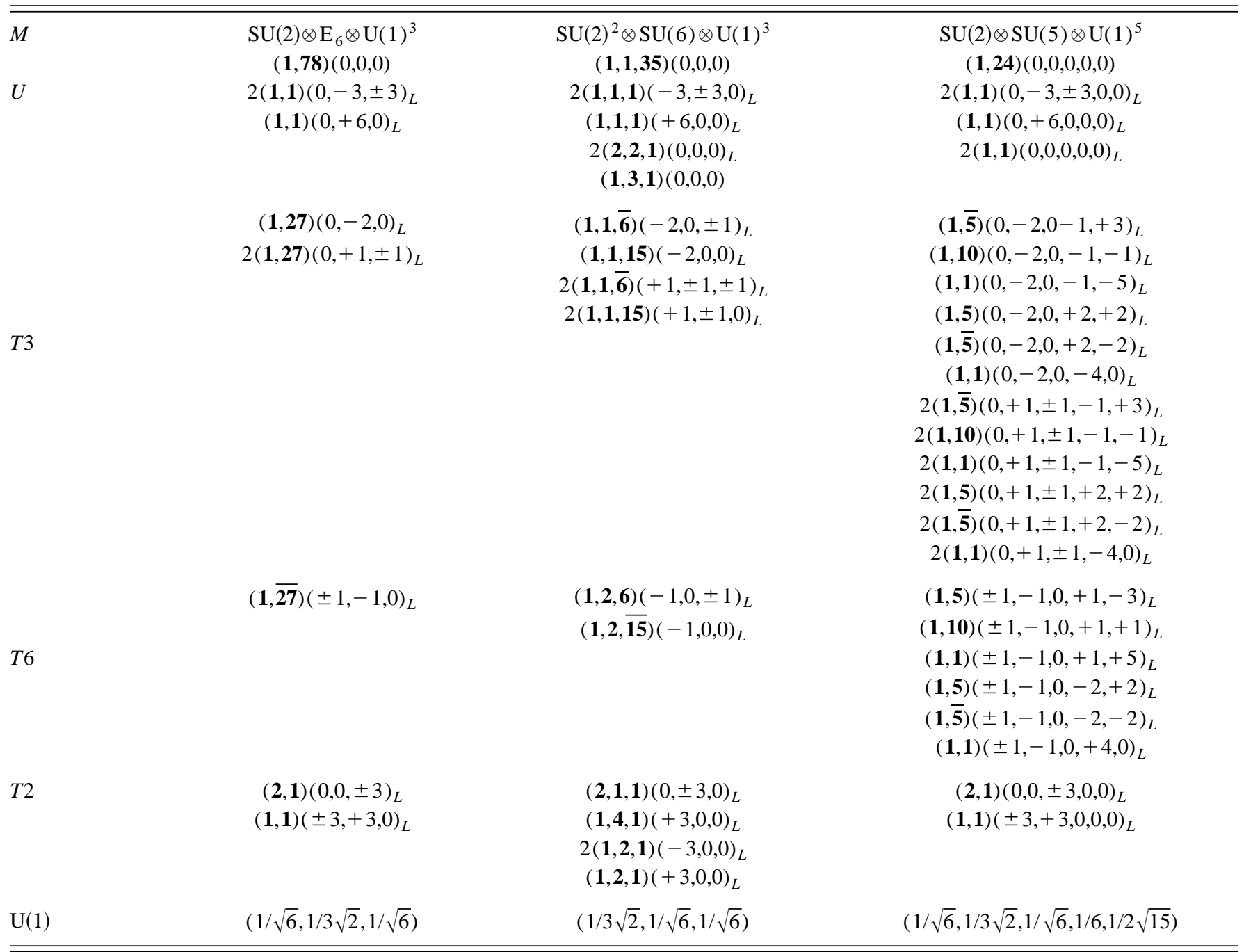

smaller intermediate sectors. Their massless spectra are given in Table II. The second $\mathrm{SO}(10)$ model is obtained by a slightly different $\mathbf{Z}_{6}$ orbifold. Although it has the same gauge symmetry as the first $\mathrm{SO}(10)$ model, it has a different spectrum. This model is discussed in Sec. VII, while its massless spectrum is shown in the second column in Table I. Section VIII discusses the third SO(10) model, whose massless spectrum is shown in the third column in Table I. The third $\mathrm{SO}(10)$ model is intimately related to the $\mathrm{E}_{6}$ model, which is discussed in Sec. IX. There we also discuss how SU(5), SU(6), and other models may be obtained from the above models. Section X contains the summary and remarks. Some of the details are relegated to the appendixes.

\section{PRELIMINARIES}

String models are constructed by considering consistent one-loop partition functions, which are built of particular linear combinations of characters, which in turn are products of characters of world-sheet free fermions and (un)twisted chiral bosons. After a general discussion of the framework, the characters are reviewed. We shall discuss the particular form of the bosonic characters that is suitable for our purpose.

\section{A. Framework}

In this subsection we set up the framework for the remainder of this paper. To be specific, we consider heterotic strings compactified to four space-time dimensions. In the lightcone gauge, which we adopt, we have the following worldsheet degrees of freedom: one complex boson $\phi^{0}$ (corresponding to two transverse space-time coordinates); three right-moving complex bosons $\phi_{R}^{\prime}, \ell=1,2,3$ (corresponding to six internal coordinates); four right-moving complex fermions $\psi^{r}, r=0,1,2,3$ ( $\psi^{0}$ is the world-sheet superpartner of the right-moving component of $\phi^{0}$, whereas $\psi$ are the world-sheet superpartners of $\left.\phi_{R}^{\prime}, \ell=1,2,3\right) ; 11$ left-moving complex bosons $\phi_{L}^{\ell}, \ell=4,5, \ldots, 14$ (corresponding to 22 internal coordinates). Before orbifolding, the corresponding string model has $N=4$ space-time supersymmetry and the internal momenta span an even self-dual Lorentzian lattice $\Gamma^{6,22}$.

It is convenient to organize the string states into sectors labeled by the monodromies of the string degrees of freedom. Thus, consider the sector where

$$
\psi^{r}\left(\overline{z e}^{-2 \pi i}\right)=\exp \left(-2 \pi i V_{i}^{r}\right) \psi^{r}(\bar{z}),
$$




$$
\begin{gathered}
\phi_{R}^{\ell}\left(\bar{z} e^{-2 \pi i}\right)=\exp \left(-2 \pi i T_{i}^{\ell}\right) \phi_{R}^{\ell}(\bar{z})-U_{i}^{\ell}, \quad \ell=1,2,3, \\
\phi_{L}^{\ell}\left(z e^{2 \pi i}\right)=\exp \left(-2 \pi i T_{i}^{\ell}\right) \phi_{L}^{\ell}(z)+U_{i}^{\ell}, \quad \ell=4, \ldots, 14 .
\end{gathered}
$$

[Note that $\phi^{0}\left(z e^{2 \pi i}, \bar{z} e^{-2 \pi i}\right)=\phi^{0}(z, \bar{z})$ since $\phi^{0}$ corresponds to space-time coordinates.] These monodromies can be combined into a single vector

$$
\begin{aligned}
V_{i}= & \left\{V_{i}^{0}\left[V_{i}^{1}\left(T_{i}^{1}, U_{i}^{1}\right)\right]\left[V_{i}^{2}\left(T_{i}^{2}, U_{i}^{2}\right)\right]\left[V_{i}^{3}\left(T_{i}^{3}, U_{i}^{3}\right)\right]\right. \\
& \left.\times \|\left(T_{i}^{4}, U_{i}^{4}\right) \cdots\left(T_{i}^{14}, U_{i}^{14}\right)\right\} .
\end{aligned}
$$

The double vertical line separates the right and left movers. Without loss of generality we can restrict the values of $V_{i}^{r}$ and $T_{i}^{\ell}$ as follows: $-\frac{1}{2} \leqslant V_{i}^{r}<\frac{1}{2}, 0 \leqslant T_{i}^{\ell}<1$. So $V_{i}^{r}$ $=0\left(-\frac{1}{2}\right)$ corresponds to a Neveu-Schwarz (NS) (Ramond) fermion. [A complex boson (fermion) with boundary condition $T_{i}^{\ell}\left(V_{i}^{r}\right)=0$ or $\frac{1}{2}$ can be split into two real bosons (fermions).] The shifts $U_{i}^{\ell}$ can be combined into a real (6,22)-dimensional Lorentzian vector $\vec{U}_{i}$ defined up to the identification $\vec{U}_{i} \sim \vec{U}_{i}+\vec{P}$, where $\vec{P}$ is an arbitrary vector of $\Gamma^{6,22}$.

The monodromies (2.1) can be viewed as fields $\Phi$ (where $\Phi$ is a collective notation for the fields $\psi^{r}, \phi_{R}^{l}$, and $\phi_{L}^{l}$ ) being periodic $\Phi\left(z e^{2 \pi i}, \overline{z e}{ }^{-2 \pi i}\right)=\Phi(z, \bar{z})$ up to the identification $\Phi \sim g\left(V_{i}\right) \Phi g^{-1}\left(V_{i}\right)$, where $g\left(V_{i}\right)$ is an element of the orbifold group $G$. In this paper we will only consider the cases where $G$ is Abelian. For two elements $g\left(V_{i}\right)$ and $g\left(V_{j}\right)$ to commute, we must have

$$
\left[1-\exp \left(-2 \pi i T_{i}^{\ell}\right)\right] U_{j}^{\ell}-\left[1-\exp \left(-2 \pi i T_{j}^{\ell}\right)\right] U_{i}^{\ell} \in \Gamma^{6,22} .
$$

In this paper we will confine our attention to cases where $U_{i}^{\ell}=0$ if $W_{j}^{\ell} \neq 0$ and $U_{j}^{\ell}=0$ if $W_{i}^{\ell} \neq 0$. (The more general case can be conveniently considered in the framework of non-Abelian orbifolds discussed in [9].)

This leads us to a simpler form of $V_{i}$ where instead of having a double entry $\left(T_{i}^{\ell}, U_{i}^{\ell}\right)$ for each complex boson we will specify either $T_{i}^{\ell}$ (whenever $T_{i}^{\ell} \neq 0$, in which case $\left.U_{i}^{\ell}=0\right)$ or $U_{i}^{\ell}$ (whenever $U_{i}^{\ell} \neq 0$, in which case $\left.T_{i}^{\ell}=0\right)$. To keep track of whether a given entry corresponds to a twist or a shift, it is convenient to introduce auxiliary vectors

$$
W_{i}=\left[0\left(0 W_{i}^{1}\right)\left(0 W_{i}^{2}\right)\left(0 W_{i}^{2}\right) \| W_{i}^{4} \cdots W_{i}^{14}\right] .
$$

The entries $W_{i}^{\ell}$ are defined as follows: $W_{i}^{\ell}=\frac{1}{2}$ if $T_{i}^{\ell} \neq 0$; $W^{\ell}=0$, otherwise. For example,

$$
\begin{gathered}
V_{i}=\left[V_{i}^{0}\left(V_{i}^{1} T_{i}^{1}\right)\left(V_{i}^{2} T_{i}^{2}\right)\left(V_{i}^{3} T_{i}^{3}\right) \| U_{i}^{4} \cdots U_{i}^{13} T_{i}^{14}\right], \\
W_{i}=\left[0\left(0 \frac{1}{2}\right)^{3} \| 0^{10} \frac{1}{2}\right],
\end{gathered}
$$

where $T_{i}^{1}, T_{i}^{2}, T_{i}^{3}$, and $T_{i}^{14}$ correspond to the twists, $U_{i}^{4} \ldots, U_{i}^{13}$ correspond to the shifts, and $V_{i}^{r}, r=0,1,2,3$, specify the fermionic spin structures.

The notation we have introduced proves convenient in describing the sectors of a given string model based on the orbifold group $G$. For $G$ to be a finite discrete group, the element $g\left(V_{i}\right)$ must have a finite order $m_{i} \in \mathbf{N}$, i.e.,
$g^{m_{i}}\left(V_{i}\right)=1$. This implies that $V_{i}^{r}$ and $T_{i}^{\ell}$ must be rational numbers, and the shift vector $\vec{U}_{i}$ must be a rational multiple of a vector in $\Gamma^{6,22}$, that is, $m_{i} V_{i}^{r}, m_{i} T_{i}^{\ell} \in \mathbf{Z}$ and $m_{i} \vec{U}_{i}$ $\in \Gamma^{6,22}$. To describe all the elements of the group $G$, it is convenient to introduce the set of generating vectors $\left\{V_{i}\right\}$ such that $\overline{\alpha V}=\mathbf{0}$ if and only if $\alpha_{i} \equiv 0$. Here $\mathbf{0}$ is the null vector:

$$
\mathbf{0}=\left[0(00)^{3} \| 0^{11}\right] .
$$

Also, $\alpha V \equiv \sum_{i} \alpha_{i} V_{i} \quad$ the summation is defined as $\left.\left(V_{i}+V_{j}\right)^{\ell}=V_{i}^{\ell}+V_{j}^{\ell}\right], \alpha_{i}$ being integers that take values from 0 to $m_{i}-1$. The overbar notation is defined as $\overline{\alpha V} \equiv \alpha V-\Delta(\alpha)$, and the components of $\overline{\alpha V}$ satisfy $-\frac{1}{2} \leqslant \overline{\alpha V}^{r}<\frac{1}{2}, 0 \leqslant \overline{\alpha T}^{\ell}<1$; here, $\Delta^{r}(\alpha), \Delta^{\ell}(\alpha) \in \mathbf{Z}$. So the elements of the group $G$ are in one-to-one correspondence with the vectors $\overline{\alpha V}$ and will be denoted by $g(\overline{\alpha V})$. It is precisely the Abelian nature of $G$ that allows this correspondence (by simply taking all possible linear combinations of the generating vectors $V_{i}$ ).

Now we can identify the sectors of the model. They are labeled by the vectors $\overline{\alpha V}$, and in a given sector $\overline{\alpha V}$ the monodromies of the string degrees of freedom are given by $\Phi\left(z e^{2 \pi i}, \bar{z} e^{-2 \pi i}\right)=g(\overline{\alpha V}) \Phi(z, \bar{z}) g^{-1}(\overline{\alpha V})$.

$G$ is a symmetry of the Hilbert space of the original string model with $N=4$ supersymmetry compatible with the operator algebra of the underlying (super)conformal field theory. If $|\chi\rangle$ is a state in the original Hilbert space, $g(\overline{\alpha V})|\chi\rangle$ [where there must exist a representation of $g(\alpha V)$ via the vertex operators of the theory] also belongs to the same Hilbert space. One consequence of this requirement is that $G$ must (anti)commute with the (super-)Virasoro algebra. This implies the supercurrent constraint

$$
V_{i}^{\ell}+T_{i}^{\ell}=V_{i}^{0} \equiv s_{i}(\bmod 1), \quad \ell=1,2,3 .
$$

Here $s_{i}$ is the monodromy of the supercurrent $\bar{S}\left(\overline{z e}{ }^{-2 \pi i}\right)$ $=\exp \left(2 \pi i s_{i}\right) \bar{S}(\bar{z})$, which must satisfy $s_{i} \in \frac{1}{2} \mathbf{Z}$. Then the sectors with $\overline{\alpha V}^{0}=0$ give rise to space-time bosons, while the sectors with $\overline{\alpha V}^{0}=-\frac{1}{2}$ give rise to space-time fermions.

Now we may write the one-loop partition function as

$$
\mathcal{Z}=\frac{1}{\Pi_{i} m_{i}} \sum_{\alpha, \beta} \operatorname{Tr}\left[q^{H \frac{L}{\alpha V}} \bar{q}^{H \frac{R}{\alpha V}} g^{-1}(\overline{\beta V})\right]=\frac{1}{\Pi_{i} m_{i} \sum_{\alpha, \beta}} C_{\overline{\beta V}}^{\overline{\alpha V}} \mathcal{Z}_{\overline{\beta V}}^{\overline{\alpha V}}
$$

where $H \frac{L}{\alpha V}$ and $H \frac{R}{\alpha V}$ are the left- and right-moving Hamiltonians, respectively. The trace is taken over the states in Hilbert space corresponding to the sector $\overline{\alpha V}$. The sum over $\alpha$ sums all the different sectors while the sum over $\beta$ provides the appropriate Gliozzi-Scherk-Olive (GSO) projections. We shall first discuss the characters $\mathcal{Z}_{\overline{\beta V}}^{\overline{\alpha V}}$. They are constructed so that modular transformations simply permute them. Then we shall solve for the coefficients $C \frac{\overline{\alpha V}}{\beta V}$ to yield a consistent $\mathcal{Z}$. 


\section{B. Fermion and boson characters}

To derive relatively simple rules for building asymmetric orbifold models, we will confine our attention to the orbifolds with twists whose orders are co-prime numbers. [The order of a twist generated by a vector $\overline{\alpha V}$ is defined as the smallest positive integer $t(\overline{\alpha V})$, such that $\forall \ell t(\overline{\alpha V}) \overline{\beta T}$ $\in \mathbf{Z}$; note that $t(\overline{\alpha V})$ is a divisor of $m(\overline{\alpha V})$.] In this case, a given model can be viewed as being generated by a single twist $V^{*}$ of order $t^{*}=\Pi_{i} t_{i}$, such that $\overline{\alpha V}=\overline{\alpha^{*} V^{*}}$, where $\alpha^{*} / t^{*}=\Sigma_{i} \alpha_{i} / t_{i}(\bmod 1), \quad$ and $\alpha^{*}$ takes values $0,1, \ldots, t^{*}-1$. Although it is possible to work in the $V^{*}$ basis, it will prove convenient to do so in the $\left\{V_{i}\right\}$ basis.

In a given sector $\overline{\alpha V}$, the right- and left-moving Hamiltonians are given by the corresponding sums of the Hamiltonians for individual string degrees of freedom. Hilbert space in the $\overline{\alpha V}$ sector is given by the momentum states $\left|\vec{P}{ }_{\alpha V}+\alpha \vec{U}, \mathbf{n}\right\rangle$, and also the states obtained from these states by the actions of the fermion and boson creation operators (oscillator excitations). $\mathbf{n}$ is a collective notation for $n_{i}=0,1, \ldots, t_{i}-1$. In the untwisted sectors, that is, sectors $\overline{\alpha V}$ with $t(\overline{\alpha V})=1$, we have $\vec{P}_{\overline{\alpha V}} \in \Gamma^{6,22}$. In the twisted sectors $\overline{\alpha V}$ with $t(\overline{\alpha V}) \neq 1$, we have $\vec{P} \overline{\alpha V} \in \widetilde{I}(\overline{\alpha V})$, where $\widetilde{I}(\overline{\alpha V})$ is the lattice dual to the lattice $I(\overline{\alpha V})$, which in turn is the sublattice of $\Gamma^{6,22}$ invariant under the action of the twist part of the group element $g(\overline{\alpha V})$. The ground states $|\overrightarrow{0}, \mathbf{n}\rangle$ in the $\overline{\alpha V}$ sector appear with certain degeneracies $\xi(\overline{\alpha V}, \mathbf{n})$, which are non-negative integers. In the untwisted sectors $\xi(\overline{\alpha V}, \mathbf{n})=1$ if $\mathbf{n}=\mathbf{0}$, and zero otherwise. In the twisted sectors the situation is more involved. First, the sum of $\xi(\overline{\alpha V}, \mathbf{n})$ over all $\mathbf{n}$ is the number of fixed points $\xi(\overline{\alpha V})$ in the $\overline{\alpha V}$ sector [2]:

$$
\sum_{\mathbf{n}} \xi(\overline{\alpha V}, \mathbf{n})=\xi(\overline{\alpha V})=[M(\overline{\alpha V})]^{-1 / 2} \prod_{\ell}[2 \sin (\pi \overline{\alpha T})],
$$

where the product over $\ell$ does not include the terms with $\overline{\alpha T}^{\ell}=0$, and $M(\overline{\alpha V})$ is the determinant of the metric of $I(\overline{\alpha V})$. Second, since different fixed points generically have different phases (monodromies) under the action of twists, the states corresponding to different fixed points contribute with different phases into the partition function. These contributions are related by the requirements of modular invariance and physically sensible projection. These constraints unambiguously fix the degeneracies $\xi(\overline{\alpha V}, \mathbf{n})$, and we will give the procedure to determine them in a moment.

Since the momentum in the twisted sectors belongs to a shifted $\widetilde{I}(\overline{\alpha V})$ lattice, to satisfy the level-matching condition, this lattice must have a prime $N(\overline{\alpha V})$, where $N(\overline{\alpha V})$ is the smallest positive integer such that for all vectors $\vec{P} \in \widetilde{I}(\overline{\alpha V}), N(\overline{\alpha V}) \vec{P}^{2} \in 2 \mathbf{Z}$; moreover, for the corresponding characters to have the correct modular transformation properties (i.e., so that they are permuted under both $S$ and $T$ transformations), either $N(\overline{\alpha V})=1$ [in which case $I(\overline{\alpha V})$ is an even self-dual lattice] or $N(\overline{\alpha V})=t(\overline{\alpha V})$ [in which case $I(\overline{\alpha V})$ is even but not self-dual].

Now the characters $\mathcal{Z}_{\overline{\beta V}}^{\overline{\alpha V}}$ can be computed as products of building blocks or contributions of individual string degrees of freedom, which are reviewed in Appendix A. The result can be written as a product of the corresponding fermion and boson characters:

$$
\mathcal{Z}_{\overline{\beta V}}^{\overline{\alpha V}}=\bar{Z} \overline{\frac{\alpha V}{\beta V}} \mathcal{Y} \overline{\overline{\beta V}}
$$

The fermion characters $\bar{Z}_{\bar{\beta} V}^{\bar{\alpha} V}$ read

$$
\bar{Z} \overline{\overline{\alpha V}}=\prod_{r} \bar{Z}_{\overline{\beta V}^{r}}^{r}
$$

[The characters $\bar{Z}_{u}^{v}$ for a right-moving fermion are complex conjugates of the characters $Z_{u}^{v}$ for a left-moving fermion given by Eq. (A6)].

The boson characters $\mathcal{Y} \overline{\overline{\beta V}}$ read

$$
\mathcal{Y} \frac{\overline{\alpha V}}{\overline{\beta V}}=\xi \frac{\overline{\alpha V}}{\overline{\beta V}} Y \frac{\overline{\alpha V}}{\beta V} \prod_{\ell=1}^{3} \bar{X}_{\overline{\beta T}}^{\overline{\alpha T}^{\ell}} \prod_{\ell=4}^{14} X_{\frac{\overline{\alpha T}^{\ell}}{\beta T}}^{\ell} .
$$

[The characters $\bar{X}_{u}^{v}$ for a right-moving boson are complex conjugates of the characters $X_{u}^{v}$ for a left-moving boson given by Eq. (A15).] The product over $\ell$ does not include terms with $\overline{\alpha T^{\ell}}=\overline{\beta T} \bar{T}^{\ell}=0$.

The characters $Y \overline{\overline{\alpha V}}$ read

$$
\begin{aligned}
Y \overline{\overline{\alpha V}}= & \frac{1}{\eta^{d} \bar{\eta}^{d^{\prime}}} \sum_{\vec{P} \in \mathcal{I}^{*}(\overline{\alpha V}, \overline{\beta V})} q^{\left(\vec{P}^{L}+\alpha \vec{U}^{L}\right)^{2 / 2}} \bar{q}^{\left(\vec{P}^{R}+\alpha \vec{U}^{R}\right)^{2}} \\
& \times \exp \left\{-2 \pi i\left[\beta \vec{U} \cdot \vec{P}+\frac{1}{2} \nu(\overline{\alpha V}, \overline{\beta V}) \vec{P}^{2}\right]\right\},
\end{aligned}
$$

where $\vec{P}^{L}, \vec{P}^{R}$, and $\vec{U}^{L}, \vec{U}^{R}$, are the left- and right-moving parts of the momentum and shift vectors, respectively. The lattice $\mathcal{I}^{*}(\overline{\alpha V}, \overline{\beta V})$ [with Lorentzian metric $\left((-)^{d^{\prime}},(+)^{d}\right)$ ] is the sublattice of $\bar{I}(\overline{\alpha V})$ invariant under the twist part of $\overline{\beta V}$. Here, $\nu(\overline{\alpha V}, \overline{\beta V})$ is an integer taking value between 0 and $N(\overline{\alpha V})-1$, defined as

$$
\alpha^{*} \nu(\overline{\alpha V}, \overline{\beta V})=\beta^{*}[\bmod N(\overline{\alpha V})], \quad \alpha^{*} \neq 0,
$$

and $\nu(\overline{\alpha V}, \overline{\beta V}) \equiv 0$, otherwise. We note that we have included the phase $\nu(\overline{\alpha V}, \overline{\beta V}) \vec{P} \frac{2}{\alpha V} / 2$ in the above definition of $Y \overline{\overline{\alpha V}}$ so that the resulting characters have simple modular transformation properties; in particular, they are permuted under $S$ and $T$ transformations (see Appendix A for a more detailed discussion).

The fixed point phase $f_{i}(\overline{\alpha V}, \mathbf{n})$ of the $\xi(\overline{\alpha V}, \mathbf{n})$ fixed points is given by

$$
f_{i}(\overline{\alpha V}, \mathbf{n})=n_{i} / t_{i}
$$

Then the coefficients $\xi_{\overline{\beta V}}^{\overline{\alpha V}}$ in $\mathcal{Y} \frac{\overline{\alpha V}}{\beta V}$ are given by

$$
\xi \frac{\overline{\alpha V}}{\beta V}=\sum_{\mathbf{n}} \xi(\overline{\alpha V}, \mathbf{n}) \exp [-2 \pi i \beta f(\overline{\alpha V}, \mathbf{n})]
$$

Following Eq. (2.10), we see that $\xi \overline{\alpha V}=\xi(\overline{\alpha V})$ for $t(\overline{\beta V})=1$. Note that $\overline{\xi \overline{\alpha V}}$ are nonzero integers. 

$\overline{\alpha V}$,

Now we can collect the phases in $\mathcal{Z}_{\overline{\beta V}}^{\overline{\alpha V}}$ in a given sector

$$
\mathcal{Z}_{\overline{\beta V}}^{\overline{\alpha V}} \equiv \operatorname{Tr}\left[q^{H \frac{L}{\alpha V}} q^{H \frac{R}{\alpha V}} h^{-1}(\overline{\beta V})\right],
$$

where $g^{-1}(\overline{\beta V})=C_{\overline{\beta V}}^{\overline{\alpha V}} h^{-1}(\overline{\beta V})$, and

$$
\begin{aligned}
h^{-1}(\overline{\beta V})= & \exp \left\{-2 \pi i\left[\beta V \cdot \mathcal{N}_{\overline{\alpha V}}+\beta f(\overline{\alpha V}, \mathbf{n})\right.\right. \\
& \left.\left.+\frac{1}{2} \nu(\overline{\alpha V}, \overline{\beta V}) \vec{P}_{\overline{\alpha V}}^{2}\right]\right\} .
\end{aligned}
$$

Here $\mathcal{N}_{\overline{\alpha V}}=\left(N_{\overline{\alpha V}}^{r}, J_{\overline{\alpha V}}^{\ell}, \vec{P} \overline{\alpha V}\right)$; i.e., $\mathcal{N}_{\overline{\alpha V}}$ is a Lorentzian vector whose components are the right-moving complex fermion number operator $N \frac{\ell}{\alpha V}$ the generators of twists $J_{\overline{\alpha V}}^{\ell}$ and the shifts $\vec{P}_{\overline{\alpha V}}$ (see Appendix A). Note that both operators $N \frac{\ell}{\alpha V}$ and $J_{\alpha V}^{\ell}$ are normalized to have integer eigenvalues. The dot product is understood to have the signature

$$
\begin{aligned}
\beta V \cdot \mathcal{N}_{\overline{\alpha V}} \equiv & \beta \vec{U} \cdot \vec{P} \overline{\alpha V}-\sum_{r}(\beta V)^{r} N_{\overline{\alpha V}}^{r}+\sum_{\ell \text { :right }}(\beta T)^{\ell} J_{\overline{\alpha V}}^{\ell} \\
& -\sum_{\ell: \text { left }}(\beta T)^{\ell} J_{\overline{\alpha V}}^{\ell} .
\end{aligned}
$$

The dot product of the vectors $\beta \vec{U}$ and $\vec{P} \overline{\alpha V}$ has the Lorentzian metric $\operatorname{diag}\left((-)^{6},(+)^{22}\right)$.

Now, the above characters are designed to have relatively simple modular transformation properties. Thus, various $\xi \frac{\overline{\alpha V}}{\beta V}$ are defined as

$$
\begin{gathered}
\xi_{\overline{\beta V}}^{\overline{\alpha V}}=\xi_{-\alpha V}^{\overline{\beta V}} \exp [2 \pi i f(\overline{\alpha V}, \overline{\beta V})]\left\{1+[M(\overline{\alpha V}, \overline{\beta V})]^{-1 / 2} \delta_{\alpha^{*}, 0}\left(1-\delta_{\beta^{*}, 0}\right)+[M(\overline{\alpha V}, \overline{\beta V})]^{1 / 2} \delta_{\beta^{*}, 0}\left(1-\delta_{\alpha^{*}, 0}\right)\right\} \\
\times \prod_{\ell}\left\{1+\left[2 \sin \left(\pi \overline{\alpha T^{\ell}}\right)\right] \delta_{\overline{\beta T^{\prime}, 0}}+\left[2 \sin \left(\pi \overline{\beta T^{\prime}}\right)\right]^{-1} \delta_{\overline{\alpha T^{\prime}, 0}}\right\} \\
\xi_{\overline{\beta V}}^{\overline{\alpha V}}=\xi_{\overline{\beta V-\alpha V+V_{0}}}^{\overline{\alpha V}}
\end{gathered}
$$

where the product over $\ell$ does not include terms with $\overline{\alpha T}^{\ell}=\overline{\beta T}^{\ell}=0 . M(\overline{\alpha V}, \overline{\beta V})$ is the determinant of the metric of $\mathcal{I}(\overline{\alpha V}, \overline{\beta V})$ which is the sublattice of $I(\overline{\alpha V})$ invariant under the twist part of $\overline{\beta V}$; note that in general $\widetilde{\mathcal{I}}(\overline{\alpha V}, \overline{\beta V})$ $\neq \mathcal{I}^{*}(\overline{\alpha V}, \overline{\beta V})$.

Since $\xi \frac{\overline{\alpha V}}{\beta V}$ are nonzero integers, the phases $f(\overline{\alpha V}, \overline{\beta V})$ are zero or $1 / 2(\bmod 1)$. The above relations and the requirement that $\xi(\overline{\alpha V}, \mathbf{n})$ be non-negative integers then unambiguously fix the phases $f(\overline{\alpha V}, \overline{\beta V})$ and degeneracies $\xi(\overline{\alpha V}, \mathbf{n})$.

The above relations between different $\frac{\xi \overline{\alpha V}}{\overline{\alpha V}}$ are such that under the $S$ - and $T$-modular transformations the characters $\mathcal{Z}_{\overline{\beta V}}^{\overline{\alpha V}}$ transform as

$$
\begin{gathered}
\mathcal{Z}_{\overline{\beta V}}^{\overline{\alpha V}} \stackrel{S}{\rightarrow} \exp [2 \pi i \varphi(\overline{\alpha V}, \overline{\beta V})] \mathcal{Z}_{-\alpha V}^{\overline{\beta V}}, \\
\mathcal{Z}_{\overline{\beta V}} \stackrel{T}{\rightarrow} \exp \left\{2 \pi i\left[\frac{1}{2} \varphi(\overline{\alpha V}, \overline{\alpha V})\right]+\frac{1}{2}\right\} \mathcal{Z}_{\overline{\beta V}-\alpha V+V_{0}}^{\overline{\alpha V}} .
\end{gathered}
$$

Here $V_{0}$ is the vector with $-1 / 2$ (i.e., Ramond) entry for each world-sheet fermion and zero otherwise:

$$
V_{0}=\left[-\frac{1}{2}\left(-\frac{1}{2} 0\right)^{3} \| 0^{11}\right] .
$$

According to the above modular transformation properties of $\mathcal{Z}_{\overline{\beta V}}^{\overline{\alpha V}}, V_{0}$ is always among the generating vectors $\left\{V_{i}\right\}$.

The phases $\varphi(\overline{\alpha V}, \overline{\beta V})$ are defined as

$$
\begin{aligned}
\varphi(\overline{\alpha V}, \overline{\beta V})= & {[\overline{\alpha V}-W(\overline{\alpha V})][\overline{\beta V}-W(\overline{\beta V})]+f(\overline{\alpha V}, \overline{\beta V}) } \\
& +\chi(\overline{\alpha V}, \overline{\beta V}),
\end{aligned}
$$

where $\chi(\overline{\alpha V}, \overline{\beta V})$ phases are defined in Appendix A. The dot product of two vectors $\overline{\alpha V}$ and $\overline{\beta V}$ is defined as in Eq. (2.20). For example,

$$
V_{i} \cdot V_{j}=\vec{U}_{i} \cdot \vec{U}_{j}-\sum_{r} V_{i}^{r} V_{j}^{r}+\sum_{\ell \text { :right }} T_{i}^{\ell} T_{j}^{\ell}-\sum_{\ell: \text { left }} T_{i}^{\ell} T_{j}^{\ell}
$$

and there is a factor of $\frac{1}{2}$ for real fermions or twisted real bosons.

\section{ORBIFOLD RULES}

In this section we derive the rules for constructing consistent string models in the framework discussed in Sec. II. (This derivation for a special case of asymmetric orbifolds was given in [12].) The contribution to the orbifold one-loop partition function is a linear combination of the characters $\mathcal{Z}_{\overline{\beta V}}^{\overline{\alpha V}}$ given above. Now we are ready to consider the coefficients $C \frac{\overline{\alpha V}}{\beta V}$ in $\mathcal{Z}$, Eq. (2.9).

The coefficients $C_{\overline{\beta V}}^{\overline{\alpha V}}$ must be such that $\mathcal{Z}$, Eq. (2.9), is modular invariant. Taking into account the modular transformation properties (2.23), we have the following constraints on the coefficients $C \frac{\overline{\alpha V}}{\beta V}$ coming from the requirement of modular invariance of Eq. (2.9):

$$
\begin{gathered}
S: C_{\overline{\beta V}}^{\overline{\alpha V}} \exp [2 \pi i \varphi(\overline{\alpha V} \cdot \overline{\beta V})]=C_{=\overline{\alpha V}}^{\overline{\beta V}}, \\
T: C_{\overline{\beta V}}^{\overline{\alpha V}} \exp \left[2 \pi i\left(\frac{1}{2} \varphi(\overline{\alpha V}, \overline{\beta V})+\frac{1}{2}\right)\right]=C_{\overline{\beta V}-\alpha V+V_{0}}^{\overline{\alpha V}} .
\end{gathered}
$$


In addition to Eqs. (3.1) and (3.2) we require that, for any physical sector labeled by $\overline{\alpha V}$, the sum over $\beta$ 's in Eq. (2.9) form a proper projection with multiplicity $\xi(\overline{\alpha V}, \mathbf{n})$. Specifically, this means that

$$
\frac{1}{\Pi_{i} m_{i}} \sum_{\beta} C_{\overline{\beta V}}^{\overline{\alpha V}} g^{-1}(\overline{\beta V})=e^{2 \pi i \alpha s} \xi(\overline{\alpha V}, \mathbf{n}) \eta\left(\overline{\alpha V}, \mathcal{N}_{\overline{\alpha V}}, \mathbf{n}\right),
$$

where $s_{i}$ is given by Eq. (2.8). Now, $\eta\left(\overline{\alpha V}, \mathcal{N}_{\overline{\alpha V}}, \mathbf{n}\right)$ takes the value 0 (i.e., projected out) or 1 (i.e., kept), depending on the values of $\alpha_{i}, \mathcal{N}_{\overline{\alpha V}}, \mathbf{n}$. This is precisely the physically sensible projection; space-time bosons contribute to the partition function with the weight +1 , whereas space-time fermions contribute with the weight -1 , each with degeneracy $\xi(\overline{\alpha V}, \mathbf{n})$ due to the number of fixed points in the twisted sectors. As a consequence, $\mathcal{Z}$ becomes

$$
\mathcal{Z}=\sum_{\alpha} \operatorname{Tr}\left[q^{H \frac{L}{\alpha V}} q^{H H} \frac{R}{\alpha V} e^{2 \pi i \alpha s} \xi(\overline{\alpha V}, \mathbf{n}) \eta\left(\overline{\alpha V}, \mathcal{N}_{\overline{\alpha V}}, \mathbf{n}\right)\right]
$$

The formal solution to Eq. (3.3) is given by

$$
C_{\overline{\beta V}}^{\overline{\alpha V}}=\exp \{2 \pi i[\beta \phi(\overline{\alpha V})+\alpha s]\},
$$

where the phases $\phi_{i}(\overline{\alpha V})$ are such that

$$
m_{i} \phi_{i}(\overline{\alpha V})=0(\bmod 1) .
$$

The phases $\phi_{i}(\overline{\alpha V})$ are constrained due to Eqs. (3.1) and (3.2):

$$
S: \beta \phi(\overline{\alpha V})+\alpha \phi(\overline{\beta V})+\alpha s+\beta s+\varphi(\overline{\alpha V}, \overline{\beta V})=0(\bmod 1),
$$

$$
T: \alpha \phi(\overline{\alpha V})+\phi_{0}(\overline{\alpha V})+\varphi(\overline{\alpha V}, \overline{\alpha V})+\frac{1}{2}=0(\bmod 1) .
$$

Let us introduce

$$
\phi_{j}\left(V_{i}\right) \equiv-k_{i j}-s_{j} .
$$

Then the general solution to Eq. (3.7) is given by

$$
\phi_{i}(\overline{\alpha V})=\sum_{j} k_{i j} \alpha_{j}+s_{i}-\varphi\left(V_{i}, \overline{\alpha V}\right)(\bmod 1),
$$

and the constraints

$$
\begin{gathered}
k_{i j}+k_{j i}=\varphi\left(V_{i}, V_{j}\right)(\bmod 1), \\
\varphi(\overline{\alpha V}, \overline{\beta V})=\sum_{i}\left[\alpha_{i} \varphi\left(V_{i}, \overline{\beta V}\right)+\beta_{i} \varphi\left(V_{i}, \overline{\alpha V}\right)\right] \\
-\sum_{i, j} \alpha_{i} \beta_{j} \varphi\left(V_{i}, V_{j}\right)(\bmod 1) .
\end{gathered}
$$

For Eq. (3.10) to be compatible with Eq. (3.8), the following constraints must also be satisfied:

$$
k_{i i}+k_{i 0}+s_{i}-\frac{1}{2} \varphi\left(V_{i}, V_{i}\right)=0(\bmod 1),
$$

$$
\begin{aligned}
\frac{1}{2} \varphi(\overline{\alpha V}, \overline{\alpha V})= & \sum_{i} \alpha_{i} \varphi\left(V_{i}, \overline{\alpha V}\right)-\frac{1}{2} \sum_{i, j} \alpha_{i} \alpha_{j} \varphi\left(V_{i}, V_{j}\right) \\
& +V_{0} \cdot \Delta(\overline{\alpha V})(\bmod 1) .
\end{aligned}
$$

Finally, let us rewrite the constraint (3.6) in terms of $k_{i j}$ and $\varphi(\overline{\alpha V}, \overline{\beta V})$ :

$$
\begin{gathered}
k_{i j} m_{j}=0(\bmod 1), \\
m_{i}\left(\varphi\left(V_{i}, \overline{\alpha V}\right)-\sum_{j} \varphi\left(V_{i}, V_{j}\right) \alpha_{j}\right)=0(\bmod 1) .
\end{gathered}
$$

Note that not all of the above constraints are independent. Thus, Eqs. (3.13) and (3.15) imply Eq. (3.11) for $i=j$; Eq. (3.14) implies Eq. (3.12) for $\alpha_{i} \equiv \beta_{i}$.

Let us summarize the above constraints. The structure constants $k_{i j}$ must satisfy the constraints

$$
\begin{gathered}
k_{i j}+k_{j i}=\varphi\left(V_{i}, V_{j}\right)(\bmod 1), \quad i \neq j, \\
k_{i i}+k_{i 0}+s_{i}-\frac{1}{2} \varphi\left(V_{i}, V_{i}\right)=0(\bmod 1), \\
k_{i j} m_{j}=0(\bmod 1) .
\end{gathered}
$$

Note that there is no summation over repeated indices here. The phases $\varphi(\overline{\alpha V}, \overline{\beta V})$ must be such that $[\overline{\alpha V} \neq \overline{\beta V}$ in Eq. (3.20)]

$$
\begin{aligned}
\varphi(\overline{\alpha V}, \overline{\beta V})= & \sum_{i}\left[\alpha_{i} \varphi\left(V_{i}, \overline{\beta V}\right)+\beta_{i} \varphi\left(V_{i}, \overline{\alpha V}\right)\right] \\
& -\sum_{i, j} \alpha_{i} \beta_{j} \varphi\left(V_{i}, V_{j}\right)(\bmod 1)
\end{aligned}
$$

$$
\begin{aligned}
& \frac{1}{2} \varphi(\overline{\alpha V}, \overline{\alpha V})= \sum_{i} \alpha_{i} \varphi\left(V_{i}, \overline{\alpha V}\right)-\frac{1}{2} \sum_{i, j} \alpha_{i} \alpha_{j} \varphi\left(V_{i}, V_{j}\right) \\
&+V_{0} \cdot \Delta(\overline{\alpha V})(\bmod 1), \\
& m_{i}\left(\varphi\left(V_{i}, \overline{\alpha V}\right)-\sum_{j} \varphi\left(V_{i}, V_{j}\right) \alpha_{j}\right)=0(\bmod 1) .
\end{aligned}
$$

Next we turn to identifying the spectrum of states. All the states are projected out of the sum in Eq. (2.9) except those satisfying

$$
\begin{aligned}
V_{i} \cdot \mathcal{N}_{\overline{\alpha V}}+f_{i}(\overline{\alpha V}, \mathbf{n})+\frac{\alpha^{*} t^{*}}{2 t_{i}} \vec{Q}^{2}(\vec{P} \overline{\alpha V}) \\
\quad=\sum_{j} k_{i j} \alpha_{j}+s_{i}-\varphi\left(V_{i}, \overline{\alpha V}\right)(\bmod 1) .
\end{aligned}
$$

This is the spectrum-generating formula. The definition of $\vec{Q}(\vec{P} \overline{\alpha V})$ will be given in a moment.

The above rules can be simplified if 


$$
\begin{aligned}
& W(\overline{\alpha V}) \cdot W(\overline{\beta V})+W(\overline{\alpha V}) \cdot \Delta(\overline{\beta V})+\Delta(\overline{\alpha V}) \cdot W(\overline{\beta V}) \\
& +f(\overline{\alpha V}, \overline{\beta V})+\chi(\overline{\alpha V}, \overline{\beta V})=0 .
\end{aligned}
$$

In this case $\varphi(\overline{\alpha V}, \overline{\beta V})$ becomes

$$
\varphi(\overline{\alpha V}, \overline{\beta V})=\overline{\alpha V} \cdot \overline{\beta V}+\alpha V \cdot W(\overline{\beta V})+\beta V \cdot W(\overline{\alpha V}) .
$$

This simplification allows us to rewrite the rules as follows. First, the structure constants must satisfy the constraints (after a redefinition of the structure constants $k_{i j} \rightarrow k_{i j}$ $\left.+W_{i} \cdot V_{j}\right)$

$$
\begin{gathered}
k_{i j}+k_{j i}=V_{i} \cdot V_{j}(\bmod 1), \quad i \neq j, \\
k_{i i}+k_{i 0}+s_{i}-\frac{1}{2} V_{i} \cdot V_{i}=0(\bmod 1), \\
\left(k_{i j}-W_{i} \cdot V_{j}\right) m_{j}=0(\bmod 1) .
\end{gathered}
$$

The constraints (3.20), (3.21), and (3.22) are also simplified to

$$
m_{i} V_{i} \cdot\left(W(\overline{\alpha V})-\sum_{j} \alpha_{j} W_{j}\right)=0(\bmod 1)
$$

and the spectrum-generating formula now reads

$$
\begin{aligned}
V_{i} \cdot \mathcal{N}_{\overline{\alpha V}}+f_{i}(\overline{\alpha V}, \mathbf{n})+\frac{\alpha^{*} t^{*}}{2 t_{i}} \vec{Q}^{2}(\vec{P} \overline{\alpha V}) \\
\quad=\sum_{j} k_{i j} \alpha_{j}+s_{i}-V_{i} \cdot[\overline{\alpha V}-W(\overline{\alpha V})](\bmod 1) .
\end{aligned}
$$

Here $\vec{Q}(\vec{P} \overline{\alpha V}) \in \widetilde{I}(\overline{\alpha V})$ is an arbitrary vector such that $\vec{P} \overline{\alpha V}-\alpha^{*} \vec{Q}(\vec{P} \overline{\alpha V}) \in I(\overline{\alpha V})$. Also recall that

$$
\begin{aligned}
V_{i} \cdot \mathcal{N}_{\overline{\alpha V}} \equiv & \vec{U}_{i} \cdot \vec{P} \overline{\alpha V}-\sum_{r} V_{i}^{r} N_{\overline{\alpha V}}^{r}+\sum_{\text {:right }} T_{i}^{\ell} J_{\overline{\alpha V}}^{\ell} \\
& -\sum_{\ell \text { left }} T_{i}^{\ell} J_{\overline{\alpha V}}^{\ell} .
\end{aligned}
$$

Note that in the twisted sectors $[t(\overline{\alpha V}) \neq 1]$, the states with quantum numbers $\mathbf{n}$ appear with the multiplicity $\xi(\overline{\alpha V}, \mathbf{n})$.

The states that satisfy the spectrum-generating formula include both on- and off-shell states. The on-shell states must satisfy the additional constraint that the left- and rightmoving energies be equal. In the $\overline{\alpha V}$ sector they are given by

$$
\begin{aligned}
& E_{\overline{\alpha V}}^{L}=-1+\sum_{\ell: \text { left }}\left\{\frac{1}{2} \overline{\alpha T}^{\ell}\left(1-\overline{\alpha T}^{\ell}\right)+\sum_{q=1}^{\infty}\left[\left(q+\overline{\alpha T^{\ell}}-1\right) n_{q}^{\ell}+\left(q-\overline{\alpha T^{\ell}}\right) \overline{n_{q}}\right]\right\} \\
& +\sum_{q=1}^{\infty} q\left(n_{q}^{0}+\bar{n}_{q}^{0}\right)+\frac{1}{2}\left(\vec{P}^{L} \frac{L}{\alpha V}+\alpha \vec{U}^{L}\right)^{2} \\
& E_{\overline{\alpha V}}^{R}=-\frac{1}{2}+\sum_{\ell \text { right }}\left\{\frac{1}{2} \overline{\alpha T}^{\ell}\left(1-\bar{\alpha} T^{\ell}\right)+\sum_{q=1}^{\infty}\left[\left(q+\overline{\alpha T}^{\ell}-1\right) m_{q}^{\ell}+\left(q-\overline{\alpha T}^{\ell}\right) \bar{m}_{q}^{\ell}\right]\right\}+\sum_{q=1}^{\infty} q\left(m_{q}^{0}+\bar{m}_{q}^{0}\right) \\
& +\frac{1}{2}\left(\vec{P}_{\overline{\alpha V}}^{R}+\alpha \vec{U}^{R}\right)^{2}+\sum_{r}\left\{\frac{1}{2}\left(\overline{\alpha V}^{r}\right)^{2}+\sum_{q=1}^{\infty}\left[\left(q+\overline{\alpha V^{r}}-\frac{1}{2}\right) k_{q}^{r}+\left(q-\overline{\alpha V^{r}}-\frac{1}{2}\right) \bar{k}_{q}^{r}\right]\right\} .
\end{aligned}
$$

Here $n_{q}^{\ell}$ and $\vec{n}_{q}$ are occupation numbers for the left-moving bosons $\phi_{L}^{\ell}$, whereas $m_{q}^{\ell}$ and $\bar{m}_{q}^{\ell}$ are those for the rightmoving bosons $\phi_{R}^{\prime}$. These take non-negative integer values. $k_{q}^{r}$ and $\overline{k_{q}^{r}}$ are the occupation numbers for the right-moving fermions, and they take only two values: 0 and 1 . The occupation numbers are directly related to the boson and fermion number operators. For example, $N_{\overline{\alpha V}}^{r}=\sum_{q=1}^{\infty}\left(k_{q}^{r}-\overline{k_{q}^{r}}\right)$; also (for the left-moving degrees of freedom, for example), $J_{\overline{\alpha V}}^{\ell}=\delta_{\overline{\alpha T}, 0} \mathcal{J}^{\ell}-\Sigma_{q=1}^{\infty}\left(n_{q}^{\prime}-\bar{n}_{q}^{\prime}\right)$, where $\mathcal{J}^{\ell}$ is the angular momentum operator acting on the momentum states.

Let us summarize the rules. To construct a consistent orbifold model, start with a $N=4$ space-time supersymmetric four-dimensional heterotic string model [13] with the internal momenta spanning an even self-dual lattice $\Gamma^{6,22}$ that possesses a $G=\otimes_{i} \mathbf{Z}_{t_{i}}$ symmetry ( $t_{i}$ are co-primes). The sublattice $I_{i} \in \Gamma^{6,22}$, invariant under the $\mathbf{Z}_{t_{i}}$ twist, must be such that $N_{i}=1$ or $t_{i}$. Now one can introduce a set of vectors $V_{i}$ (which includes $V_{0}$ ) that correspond to a particular embedding of the orbifold group $G$. Each $\left\{V_{i}\right\}$ must satisfy Eq. (2.8). If we can find a set $\left\{V_{i}, k_{i j}\right\}$ that satisfies the constraints (3.26), (3.27), (3.28), and (3.29), the constraint (3.24) follows automatically and we have a consistent orbifold model. The complete spectrum (on- and off-shell states) of the model is given by the spectrum-generating formula (3.30), which together with the left or right energy formulas (3.32) and (3.33) determine the on-shell physical spectrum. In the remaining sections, we shall use this simplified set of rules to construct various grand unified string models. Only when the set $\left\{V_{i}, k_{i j}\right\}$ does not satisfy the simplified set of constraints do we need to check if it satisfies the more general set of constraints (3.17), (3.18), (3.19), (3.20), and (3.21). We consider such an example in Appendix B. 


\section{THREE-FAMILY SO(10) ${ }_{3}$ MODEL}

In this section we shall use the simplified version of the rules derived in Sec. III to construct the model described in Ref. [11], i.e., the $\mathrm{SO}(10)_{3}$ model with three generations of chiral matter fields. We start from an $N=4$ space-time supersymmetric Narain model, which we will refer to as $N 0$, with the lattice $\Gamma^{6,22}=\Gamma^{2,2} \otimes \Gamma^{4,4} \otimes \Gamma^{16}$. Here $\Gamma^{2,2}=\left\{\left(p_{R}|| p_{L}\right)\right\}$ is an even self-dual Lorentzian lattice with $p_{R}, p_{L} \in \widetilde{\Gamma}^{2}\left[\mathrm{SU}(3)\right.$ weight lattice], and $p_{L}-p_{R} \in \Gamma^{2}[\mathrm{SU}(3)$ root lattice]. Similarly, $\Gamma^{4,4}=\left\{\left(P_{R} \| P_{L}\right)\right\}$ is an even self-dual Lorentzian lattice with $P_{R}, P_{L} \in \widetilde{\Gamma}^{4}[\mathrm{SO}(8)$ weight lattice], $P_{L}-P_{R} \in \Gamma^{4}[\mathrm{SO}(8)$ root lattice $] . \Gamma^{16}$ is the $\operatorname{spin}(32) / \mathbf{Z}_{2}$ lattice. This model has $\mathrm{SU}(3) \otimes \mathrm{SO}(8) \otimes \mathrm{SO}(32)$ gauge group. It is useful to recall that there are four irreducible representations (irreps) in the $\mathrm{SO}(2 n)$ weight lattice: the identity $\mathbf{0}$, which stands for the null vector, the vector $\mathbf{v}$, the spinor $\mathbf{s}$, and the antispinor $\overline{\mathbf{s}}$ (also known as the conjugate $\mathbf{c}$ ), with conformal dimensions $0,1 / 2, n / 8$, and $n / 8$, respectively. For $\mathrm{SO}(2)$, we shall use $0, V= \pm 1, S=1 / 2$, and $C=-1 / 2$ to designate the weights. The gauge bosons come from the $\mathbf{0}$ weight.

Next, consider the model generated by the following set of vectors acting on the $N 0$ model:

$$
\begin{gathered}
V_{0}=\left[-\frac{1}{2}\left(-\frac{1}{2} 0\right)^{3}|| 0_{r}^{3} \mid 0_{r}^{16}\right], \\
V_{1}=\left[0\left(0 \frac{e_{1}}{2}\right)\left(0 a_{1}\right)\left(0 b_{1}\right)|| 0^{3} \mid\left(\frac{1}{2}\right)_{r}^{5} 0_{r}^{5} 0_{r}^{5}\left(-\frac{1}{2}\right)_{r}\right], \\
V_{2}=\left[0\left(0 \frac{e_{2}}{2}\right)\left(0 a_{2}\right)\left(0 b_{2}\right) \| 0^{3} \mid 0_{r}^{5}\left(\frac{1}{2}\right)_{r}^{5} 0_{r}^{5}\left(-\frac{1}{2}\right)_{r}\right],
\end{gathered}
$$

where the subscript $r$ indicates real bosons. Here $e_{1}$ and $e_{2}$ are the simple roots of $\mathrm{SU}(3)\left(e_{1} \cdot e_{1}=e_{2} \cdot e_{2}=-2 e_{1} \cdot e_{2}\right.$ $=2)$, whereas the four-dimensional real vectors $\mathbf{s}^{\prime}=\left(a_{1}, b_{1}\right)$ and $\mathbf{c}^{\prime}=\left(a_{2}, b_{2}\right)$ are fixed elements in the spinor and conjugate weights of $\mathrm{SO}(8)$, respectively. The generating vectors $V_{1}$ and $V_{2}$ are order two shifts $\left(m_{1}=m_{2}=2\right)$. The auxiliary vectors $W(\overline{\alpha V})$ are therefore null as there is no twisting of the lattice. So the constraint (3.29) is trivial.

The matrix of the dot products $V_{i} \cdot V_{j}$ reads

$$
V_{i} \cdot V_{j}=\left(\begin{array}{ccc}
-1 & 0 & 0 \\
0 & 0 & 1 \\
0 & 1 & 0
\end{array}\right) \text {. }
$$

Now, consider the constraints (3.26), (3.27), and (3.28). The structure constants $k_{11}=k_{01}=k_{10}$ and $k_{22}=k_{02}=k_{20}$ must be chosen to equal zero for the supersymmetry not to be broken from $N=4$ to $N=0$. The structure constants $k_{00}$ and $k_{12}=k_{21}$ can be chosen to equal 0 or $\frac{1}{2}$. For definiteness we can choose

$$
k_{i j}=0 \text {. }
$$

So we have a consistent model, which we will refer to as $N 1$. To see its spectrum, we note that the model has eight sectors: $\alpha_{i}=0,1$, for $i=0,1,2$. Here, $\alpha_{0}=0$ corresponds to space-time bosons and $\alpha_{0}=1$ corresponds to space-time fermions. The $\mathrm{SO}(32)$ shifts are given in the $\mathrm{SO}(10)^{3} \otimes \mathrm{SO}(2)$ basis. For $\mathrm{SO}(10),\left(\frac{1}{2}\right)_{r}^{5}$ is a component in $\mathbf{s}$, while $\mathbf{v}=\left( \pm 1,0^{4}\right)$ (and its permutations). It is convenient to consider $\mathrm{SO}(32)$ weights in terms of $\mathrm{SO}(10)^{3} \otimes \mathrm{SO}(2)$ weights. Under $\mathrm{SO}(32) \supset \mathrm{SO}(10)^{3} \otimes \mathrm{SO}(2)$, we have

$$
\begin{aligned}
\mathbf{0}= & (\mathbf{0}, \mathbf{0}, \mathbf{0}, 0)+(\mathbf{v}, \mathbf{v}, \mathbf{v}, V)+(\mathbf{0}, \mathbf{0}, \mathbf{v}, V)+(\mathbf{v}, \mathbf{v}, \mathbf{0}, 0)+(\mathbf{0}, \mathbf{v}, \mathbf{0}, V) \\
& +(\mathbf{v}, \mathbf{0}, \mathbf{v}, 0)+(\mathbf{v}, \mathbf{0}, \mathbf{0}, V)+(\mathbf{0}, \mathbf{v}, \mathbf{v}, 0), \\
\mathbf{s}= & (\mathbf{s}, \mathbf{s}, \mathbf{s}, S)+(\mathbf{c}, \mathbf{c}, \mathbf{c}, C)+(\mathbf{s}, \mathbf{c}, \mathbf{c}, S)+(\mathbf{c}, \mathbf{s}, \mathbf{s}, C)+(\mathbf{c}, \mathbf{s}, \mathbf{c}, S) \\
& +(\mathbf{s}, \mathbf{c}, \mathbf{s}, C)+(\mathbf{c}, \mathbf{c}, \mathbf{s}, S)+(\mathbf{s}, \mathbf{s}, \mathbf{c}, C) .
\end{aligned}
$$

Let us first consider the $\mathbf{0}$ sector. The spectrum generating formula (3.30) becomes

$$
\begin{gathered}
V_{0} \cdot \mathcal{N}=\frac{1}{2}\left(N^{0}+N^{1}+N^{2}+N^{3}\right)=\frac{1}{2}(\bmod 1) \\
V_{1} \cdot \mathcal{N}=-\frac{1}{2} e_{1} \cdot p_{R}-\mathbf{s}^{\prime} \cdot P_{R}+\mathbf{s} \cdot P_{1}-\frac{1}{2} Q=0(\bmod 1) \\
V_{2} \cdot \mathcal{N}=-\frac{1}{2} e_{1} \cdot p_{R}-\mathbf{c}^{\prime} \cdot P_{R}+\mathbf{s} \cdot P_{2}-\frac{1}{2} Q=0(\bmod 1)
\end{gathered}
$$

where $P_{1}$ and $P_{2}$ are the momenta of the first and the second $\mathrm{SO}(10)$, respectively. Here $Q$ is the $\mathrm{SO}(2)$ weight [i.e., the $\mathrm{U}(1)$ charge] and $\mathbf{s}$ stands for the fixed component $\left(\frac{1}{2}, \frac{1}{2}, \frac{1}{2}, \frac{1}{2}, \frac{1}{2}\right)$. Let us concentrate on the massless spectrum. The right-moving vacuum energy in the $\overline{\alpha V}=\mathbf{0}$ sector is $-1 / 2$, but Eq. (4.8) indicates that at least one NS fermion must be excited. The lowest-energy states then correspond to one NS fermionic oscillator excitations that contribute $1 / 2$ to the energy, reaching $E^{R}=0 . N^{0}= \pm 1$ corresponds to a helicity state. Together with the lowest left-moving space-time oscillator excitation (i.e., $n_{1}^{0}=1$ or $\bar{n}_{1}^{0}=1$ to reach $E^{L}=0$ ), we have the graviton, the dilaton, and the axion. To obtain the gauge bosons (excluding the ones in the supergravity multiplet), we need to consider only the left-moving part of the lattices, since all $p_{R}$ and $P_{\mathrm{SO}(8) R}$ must be zero. The spectrum-generating formula removes all but the $(\mathbf{0 , 0 , 0 , 0 )}$ in the $\mathbf{0}$ of $\mathrm{SO}(32)$, which gives the gauge bosons in $\mathrm{SO}(10)^{3}$ $\otimes \mathrm{SO}(2)$. It is easy to see that all the $\mathrm{SU}(3) \otimes \mathrm{SO}(8)$ states with $E^{L}=0$ in the $N 0$ model remain intact. If we excite one of the other fermions, i.e., $N^{i}= \pm 1$ for $i=1,2$, or 3 , we obtain the scalar superpartners.

In the $\alpha_{0}=1$ (i.e., $V_{0}$ ) sector, we have the same spectrumgenerating formula as above; here, $E^{R}=0$ already, and the $N^{i}=0,1$ stands for the Ramond degeneracy. So we have $2^{4} / 2=8$ states. Together with $n_{1}^{0}=1$ or $\bar{n}_{1}^{0}=1$, this yields four gravitinos. It is equally straightforward to get the rest of the massless states in this sector and to see that the other sectors, i.e., the shifted sectors, give rise to massive states only. So the resulting $N 1$ model is a Narain model with $N=4$ space-time supersymmetry and the gauge group $\mathrm{SU}(3)$ $\otimes \mathrm{SO}(8) \otimes \mathrm{SO}(10)^{3} \otimes \mathrm{SO}(2)$. Thus, introducing the shift vectors $V_{1}$ and $V_{2}$ is equivalent to starting from the $N 0$ Narain model and turning on appropriate Wilson lines.

Next, we want to perform a $\mathbf{Z}_{6}$ orbifold on the $N 1$ model. There are at least two equivalent ways to reach the same final model. We shall present one approach here and another approach in Sec. V. Let us first give a preview of what we shall 
do. Since $\mathbf{Z}_{6}=\mathbf{Z}_{2} \otimes \mathbf{Z}_{3}$, we may consider the $\mathbf{Z}_{2}$ and $\mathbf{Z}_{3}$ orbifolds separately. Let us call the $\mathbf{Z}_{3}$ orbifold the $A 1$ model and the $\mathbf{Z}_{2}$ orbifold the $A 2$ model. Let us first describe the $\mathbf{Z}_{3}$ symmetry.

The even self-dual Lorentzian lattice $\Gamma^{\prime 6,22}$ of the $N 1$ model has a number of $\mathbf{Z}_{3}$ symmetries, (i) a $\mathbf{Z}_{3}$ symmetry under the rotation of the right-moving momenta $p_{R}$ (corresponding to the $\Gamma^{2,2}$ sublattice of the original lattice $\Gamma^{6,22}$ ) by $2 \pi / 3$, (ii) a $\mathbf{Z}_{3}$ symmetry under the permutation of the world-sheet bosons corresponding to the three $\mathrm{SO}(10)$ s, and (iii) a $\mathbf{Z}_{3} \otimes \mathbf{Z}_{3}$ symmetry of the $\Gamma^{4,4}$ sublattice of $\Gamma^{6,22}$.

To see this last symmetry explicitly, it is useful to consider $\mathrm{SO}(8)$ in its $\mathrm{SU}(3) \otimes \mathrm{U}(1) \otimes \mathrm{U}(1)$ basis:

$$
\begin{aligned}
\mathbf{8}_{v}= & \mathbf{1}(0,1)+\mathbf{1}(0,-1)+\mathbf{3}\left(\frac{1}{\sqrt{3}}, 0\right)+\overline{3}\left(-\frac{1}{\sqrt{3}}, 0\right) \\
\mathbf{8}_{s}= & \mathbf{1}\left(\frac{\sqrt{3}}{2}, \frac{1}{2}\right)+\mathbf{1}\left(-\frac{\sqrt{3}}{2},-\frac{1}{2}\right)+\mathbf{3}\left(-\frac{1}{2 \sqrt{3}}, \frac{1}{2}\right) \\
& +\overline{3}\left(\frac{1}{2 \sqrt{3}},-\frac{1}{2}\right), \\
\mathbf{8}_{c}= & \mathbf{1}\left(\frac{\sqrt{3}}{2},-\frac{1}{2}\right)+\mathbf{1}\left(-\frac{\sqrt{3}}{2}, \frac{1}{2}\right)+\mathbf{3}\left(-\frac{1}{2 \sqrt{3}},-\frac{1}{2}\right) \\
& +\overline{3}\left(\frac{1}{2 \sqrt{3}}, \frac{1}{2}\right) .
\end{aligned}
$$

Notice that the charges on the $\mathrm{U}(1) \otimes \mathrm{U}(1)$ plane have an explicit $\mathbf{Z}_{3}$ symmetry [as they lie in a rescaled $\mathrm{SU}(3)$ lattice]. Under this $\mathbf{Z}_{3}$ rotation, it is easy to see that $\mathbf{8}_{v} \rightarrow \mathbf{8}_{s} \rightarrow \mathbf{8}_{c}$, which is simply the well-known triality of the $\mathrm{SO}(8)$ Dynkin diagram. This $\mathbf{Z}_{3}$ orbifold of $\mathrm{SO}(8)$ yields the group $G_{2}$. Since the central charge of the level-one $G_{2}$ is $c=14 / 5$, the rest of the central charge is in the corresponding coset. It is easy to see that the $\mathbf{Z}_{3}$ orbifold of the $\mathrm{SO}(8)_{1}$ current algebra can be written as the product of a $\left(G_{2}\right)_{1}$ current algebra, an Ising model $(c=1 / 2)$, and a tricritical Ising model $(c=7 / 10)$. The other $\mathbf{Z}_{3}$ symmetry in $\mathrm{SO}(8)$ that we are interested in is simply that of the SU(3) lattice specified above. Under this $\mathbf{Z}_{3}$ rotation, the three states of $\mathbf{3}$ permute among themselves (similarly for the $\overline{3}$ ). This $\mathbf{Z}_{3}$ orbifold reduces $\mathrm{SO}(8)$ to $\mathrm{U}(1) \otimes \mathrm{U}(1) \otimes \mathrm{SU}(3)_{1}$. Here, the $\mathrm{SU}(3)_{1}$ in $\mathrm{Eq}$. (4.11) is reduced to $U(1) \otimes U(1)$, while the $U(1) \otimes U(1)$ there forms the Cartan basis of the new SU(3) ${ }_{1}$. Under the diagonal $\mathbf{Z}_{3}$ (i.e., simultaneous $\mathbf{Z}_{3} \otimes \mathbf{Z}_{3}$ ) orbifold, $\mathrm{SO}(8)_{1}$ goes to $\mathrm{SU}(3)_{3}$. Now we mod the $N 1$ model by the above diagonal $\mathbf{Z}_{3}$ symmetry [(i), (ii), and (iii)] to construct a model with $\mathrm{SO}(10)$ gauge subgroup realized via the corresponding current algebra at level 3. So before the $\mathbf{Z}_{2}$ twist, the gauge group of the $A 1$ model is $\mathrm{SU}(3)_{1} \otimes \mathrm{SU}(3)_{3} \otimes \mathrm{SO}(10)_{3}$ $\otimes \mathrm{U}(1)$. This model has nine generations of chiral fermions of $\mathrm{SO}(10)$. To cut the number of generations to 3 we will mod the resulting model by its $\mathbf{Z}_{2}$ symmetry that corresponds to a symmetric $\mathbf{Z}_{2}$ twist of the $\Gamma^{4,4}$ sublattice. The $\mathbf{Z}_{3}$ orbifold is asymmetric, whereas the $\mathbf{Z}_{2}$ orbifold is symmetric. Now we are ready for the explicit construction.
Let us start from the $N 1$ model and consider the asymmetric orbifold model generated by the vectors

$$
\begin{gathered}
V_{0}=\left[-\frac{1}{2}\left(-\frac{1}{2} 0\right)^{3}|| 0^{3} \mid 0^{5} 0_{r}^{5} 0_{r}\right], \\
V_{1}=\left[0\left(-\frac{1}{3} \frac{1}{3}\right)^{3} \| 0\left(\frac{1}{3}\right)^{2} \mid\left(\frac{1}{3}\right)^{5} 0_{r}^{5}\left(\frac{2}{3}\right)_{r}\right], \\
\left.V_{2}=\left[0(00)\left(-\frac{1}{2} \frac{1}{2}\right)^{2}|| \mid \frac{e_{1}}{2}\right)\left(\frac{1}{2}\right)^{2} \mid 0^{5} 0_{r}^{5} 0_{r}\right], \\
W_{1}=\left[0\left(0 \frac{1}{2}\right)^{3}|| 0\left(\frac{1}{2}\right)^{2} \mid\left(\frac{1}{2}\right)^{5} 0_{r}^{5} 0_{r}\right], \\
W_{2}=\left[0(00)\left(0 \frac{1}{2}\right)^{2}|| 0\left(\frac{1}{2}\right)^{2} \mid 0^{5} 0_{r}^{5} 0_{r}\right] .
\end{gathered}
$$

So $t_{1}=m_{1}=3, t_{2}=m_{2}=2$, and $t^{*}=6$. Here the right- and left-moving bosons corresponding to the $\mathrm{SU}(3) \otimes \mathrm{SO}(8)$ subgroup are complexified, whereas the single boson corresponding to the $\mathrm{SO}(2)$ subgroup is real. Let the real bosons $\phi_{p}^{I}, I=1, \ldots, 5$, correspond to the $p$ th $\mathrm{SO}(10)$ subgroup, $p=1,2,3$. We may recombine these 15 real bosons into five real and five complex bosons,

$$
\begin{gathered}
\varphi^{I}=\frac{1}{\sqrt{3}}\left(\phi_{1}^{I}+\phi_{2}^{I}+\phi_{3}^{I}\right), \\
\Phi^{I}=\frac{1}{\sqrt{3}}\left(\phi_{1}^{I}+\omega \phi_{2}^{I}+\omega^{2} \phi_{3}^{I}\right),
\end{gathered}
$$

where $\omega=\exp (2 \pi i / 3)$. This is the eigenbasis of the $\mathbf{Z}_{3}$ twist, and this is the basis that the above vectors act on. Thus, along with the $\mathbf{Z}_{3}$ rotations of the complex bosons corresponding to the $\mathrm{SU}(3) \otimes S O(8)$ subgroup, the orbifold group element described by the $V_{1}$ vector also permutes the real bosons corresponding to the three $\mathrm{SO}(10)$ 's, which in turn is equivalent to modding out by their outer automorphism.

The matrix of the dot products $V_{i} \cdot V_{j}$ reads

$$
V_{i} \cdot V_{j}=\left(\begin{array}{ccc}
-1 & -1 / 2 & -1 / 2 \\
-1 / 2 & -1 / 3 & -1 / 3 \\
-1 / 2 & -1 / 3 & 0
\end{array}\right) \text {. }
$$

Following from the constraints (3.26), (3.27), and (3.28), the structure constants $k_{i j}$ can be given by

$$
k_{i j}=\left(\begin{array}{ccc}
k_{00} & 0 & k_{20}+1 / 2 \\
1 / 2 & 1 / 3 & 0 \\
k_{20} & 2 / 3 & k_{20}
\end{array}\right) .
$$

For definiteness we will take $k_{00}=0$. (The other choice $k_{00}=1 / 2$ leads to the model with chirality of fermions opposite to that of the one under consideration.) Here we choose $k_{20}=1 / 2$; as we shall see, with this choice, the resulting model has $N=1$ space-time supersymmetry. (The other choice $k_{20}=0$ leads to a model with no supersymmetry.) $\left\{V_{i}, k_{i j}\right\}$ satisfies the simplified rules of Sec. III. The condition (3.24) follows automatically. [As a check, we note that $\chi(\overline{\alpha V}, \overline{\beta V})=0$ and $f(\overline{\alpha V}, \overline{\beta V})=0$.] 
We will describe the above model in two steps. First we consider the model (the $A 1$ model), generated by the vectors $\left\{V_{0}, V_{1}\right\}$ on the $N 1$ model. As we have mentioned already, it has the gauge group $\mathrm{SU}(3)_{1} \otimes \mathrm{SU}(3)_{3} \otimes \mathrm{SO}(10)_{3} \otimes \mathrm{U}(1)$ with $N=1$ supersymmetry. As we shall see, it also has three generations of scalars in the adjoint of $\mathrm{SO}(10)_{3}$ and nine generations of chiral 16, s of $\mathrm{SO}(10)_{3}$. (There are other fields in the massless spectrum which we will give in a moment.) To cut the number of generations to 3 , we introduce the $V_{2}$ vector, i.e., the $\left\{V_{0}, V_{1}, V_{2}\right\}$ model. Sometimes, it is also illuminating to consider the $\left\{V_{0}, V_{2}\right\}$ model (the $A 2$ model, i.e., $\left\{V_{0}, V_{2}\right\}$ acting on the $N 1$ model).

The space-time bosons and fermions come from the sectors $\overline{\alpha V}$ with $\alpha_{0}=0$ and $\alpha_{0}=1$, respectively. In the untwisted sectors $\overline{\alpha V}, \alpha_{0}=0,1, \alpha_{1}=0$, the spectrum-generating formula (3.30) reads

$$
\begin{aligned}
& V_{0} \cdot \mathcal{N}_{\overline{\alpha V}}=\frac{1}{2} \sum_{r=0}^{3} N \frac{r}{\alpha V}=\frac{1}{2}(\bmod 1) \\
& V_{1} \cdot \mathcal{N}_{\overline{\alpha V}}= \frac{1}{3}\left(\sum_{r=1}^{3} N N_{\overline{\alpha V}}^{r}+\sum_{\ell=1}^{3} J \frac{\ell}{\alpha V}-\sum_{\ell=5}^{11} J \frac{\ell}{\alpha V}\right)+\frac{2}{3} Q \\
&= 0(\bmod 1), \\
& V_{2} \cdot \mathcal{N}_{\overline{\alpha V}}= \frac{1}{2}\left(\sum_{r=2}^{3} N_{\frac{r}{\alpha V}}^{r}+\sum_{\ell=2}^{3} J \frac{\ell}{\alpha V}+\frac{1}{2} e_{1} \cdot p_{L}-\sum_{\ell=5}^{6} J \frac{\ell}{\alpha V}\right) \\
&= 0(\bmod 1),
\end{aligned}
$$

where $Q$ is the $\mathrm{U}(1)$ charge [i.e., the $\mathrm{SO}(2)$ weight] and $\left(0 \mid p_{L}\right) \in \Gamma^{2,2}$. The right-moving vacuum energy in the $\mathbf{0}$ sector is $-1 / 2$, but according to Eq. (4.17) at least one NS fermion must be excited. The lowest-energy states then correspond to one NS fermionic oscillator, excitations that contribute $1 / 2$ to the energy. So for the states to be massless, we must have $J_{\overline{\alpha V}}^{\ell}=0, \ell=1,2,3$; otherwise, either the rightmoving momenta are nonzero or the right-moving oscillators are excited, and the resulting states are massive in both cases. The right-moving vacuum energy in the $V_{0}$ sector is already 0 .

It is convenient to define $J_{\mathrm{SO}(8) L}=J^{5}+J^{6}$. Then, in the appropriate basis, $J_{\mathrm{SO}(8) L}$ measures the eigenphase of the twist operation on $P_{\mathrm{SO}(8) L}$ of $\Gamma^{4,4}$. Similarly, let $J_{\mathrm{SO}(10)}=\sum_{\ell=7}^{11} J^{\ell}$. So the spectrum-generating formulas for the massless states reduce to

$$
\begin{gathered}
\frac{1}{2}\left(N^{0}+N^{1}+N^{2}+N^{3}\right)=\frac{1}{2}(\bmod 1), \\
\frac{1}{3}\left(N^{1}+N^{2}+N^{3}-J_{\mathrm{SO}(8) L}-J_{\mathrm{SO}(10)}\right)+\frac{2}{3} Q=0(\bmod 1) \\
\frac{1}{2}\left(N^{2}+N^{3}+e_{1} \cdot p_{L}-J_{\mathrm{SO}(8) L}\right)=0(\bmod 1),
\end{gathered}
$$

and the left-moving energy becomes

$$
E^{L}=-1+\frac{1}{2}\left(p_{L}^{2}+P_{\mathrm{SO}(8) L}^{2}+P_{\mathrm{SO}(10)}^{2}+Q^{2}\right)+\text { oscillators. }
$$

Let us first consider the $\left\{V_{0}, V_{1}\right\}$ model, i.e., the $A 1$ model. Under the $\mathbf{Z}_{3}$ twist, $J_{\mathrm{SO}(8) L}=0,1,2(\bmod 3)$ and $J_{\mathrm{SO}(10)}$ $=0,1,2,(\bmod 3)$. To find the gauge bosons in this model, let $N^{0}= \pm 1$ in the $\mathbf{0}$ sector. States in the original root lattice of $\mathrm{SO}(10)^{3}$ give $E^{L}=0$ already, and so $Q=0$ and $P_{\mathrm{SO}(8) L}=0$. Of the three $J_{\mathrm{SO}(10)}=0,1,2$ eigenstates, only the $J_{\mathrm{SO}(10)}=0$ (i.e., $\mathbf{Z}_{3}$-invariant) states are kept by Eq. (4.21). This gives the gauge bosons of $\mathrm{SO}(10)_{3}$. Similarly, we can consider the original root lattice of $\mathrm{SO}(8)$. In the $\mathrm{SU}(3) \otimes \mathrm{U}(1) \otimes \mathrm{U}(1)$ basis, the $\mathbf{2 8}$ of $\mathrm{SO}(8)$ becomes

$$
\begin{aligned}
\mathbf{2 8}= & \mathbf{1}(0,0)+\mathbf{1}(0,0)+\mathbf{3}\left(\frac{1}{\sqrt{3}},\right)+\overline{3}\left(-\frac{1}{\sqrt{3}},-1\right) \\
& +\mathbf{3}\left(\frac{1}{\sqrt{3}},-1\right)+\overline{3}\left(-\frac{1}{\sqrt{3}}, 1\right)+\mathbf{3}\left(-\frac{2}{\sqrt{3}}, 0\right) \\
& +\overline{3}\left(\frac{2}{\sqrt{3}}, 0\right)+\mathbf{8}(0,0) .
\end{aligned}
$$

The eigenstates of the $\mathbf{Z}_{3}$ twist can be organized in the basis $\mathrm{SO}(8) \supset \mathrm{SU}(3)_{3}$,

$$
\mathbf{2 8}=\mathbf{8}+\mathbf{1 0}+\overline{10},
$$

which have $J_{\mathrm{SO}(8) L}=0,1,2$, respectively. Again, only the $J_{\mathrm{SO}(8) L}=0$ states are kept. The remaining $\mathrm{SU}(3) \otimes \mathrm{U}(1)$ gauge bosons in the original $N 1$ model are left untouched. If, instead of the $N^{0}$ excitation, we have $N^{i}= \pm 1$ for $i=1,2$ or 3 , then we have scalar fields. Then Eq. (4.21) implies either $J_{\mathrm{SO}(8) L}=1$, giving 10 of $\mathrm{SU}(3)_{3}$, or $J_{\mathrm{SO}(10)}=1$, giving $\mathrm{SO}(10)$ adjoint Higgs fields.

This model has $N=1$ space-time supersymmetry. This is easiest to see if we count the number of gravitinos in the $V_{0}$ sector. The only quantum numbers of the Ramond fermions allowed by Eqs. (4.20), and (4.21) are $\left(N^{0}, N^{1}, N^{2}, N^{3}\right)=(0,1,1,1)$ or $(1,0,0,0)$, yielding only one gravitino. Next, let us consider the chiral fields in this sector. Let us choose $N^{0}=0$. For $\left(N^{1}, N^{2}, N^{3}\right)=(1,0,0),(0,1,0)$, and $(0,0,1)$, Eq. (4.21) requires $J_{\mathrm{SO}(8) L}=1$. This gives the particle states in the $\mathbf{1 0}$ of $\mathrm{SU}(3)_{3}$. The conjugate states in the 10 come from the choice $N^{0}=1$, together with $\left(N^{1}, N^{2}, N^{3}\right)=(1,1,0),(0,1,1)$, and $(1,0,1)$. Together they form three copies of $\mathbf{1 0}_{L}$ of SU(3) ${ }_{3}$, where the left-handed chirality label is by convention. This choice of convention fixes the chiralities of the rest of the spectrum.

It is straightforward to obtain the remaining massless states in the untwisted sector of the $A 1$ model. To summarize, we have the $N=1$ supergravity multiplet together with the dilaton-axion supermultiplet, the $N=1$ Yang-Mills supermultiplet in the adjoint of $\mathrm{SU}(3)_{1} \otimes \mathrm{SU}(3)_{3} \otimes \mathrm{SO}(10)_{3}$ $\otimes \mathrm{U}(1)$, three $N=1$ chiral supermultiplets in $(\mathbf{1}, \mathbf{1 0}, \mathbf{1})(0)_{L}$ [the boldface in parentheses indicates the irreps of $\mathrm{SU}(3)_{1}$ $\otimes \mathrm{SU}(3)_{3} \otimes \mathrm{SO}(10)_{3}$, and the $\mathrm{U}(1)$ charge is given in parentheses in regular font; the subscript indicates the space-time chirality: $L$ and $R$ for left- and right-handed fermions, respectively], and three $N=1$ Higgs supermultiplets in $(\mathbf{1}, \mathbf{1}, \mathbf{4 5})(0)$.

To obtain the massless states in the untwisted sector of the final model, we now impose the $V_{2}$ constraint. The $e_{1} / 2$ shift 
in the $V_{2}$ vector breaks $\mathrm{SU}(3)_{1}$ to $\mathrm{SU}(2)_{1} \otimes \mathrm{U}(1)$, since the gauge bosons corresponding to the four roots containing an $e_{2}$ factor are removed by the constraint (4.22). Now, under the $\mathbf{Z}_{6}=\mathbf{Z}_{3} \otimes \mathbf{Z}_{2}$ twist, $J_{\mathrm{SO}(8) L}=0,1,2,3,4,5$. To see that the $\mathbf{Z}_{2}$ twist on the $\mathrm{SO}(8)$ lattice breaks $\mathrm{SU}(3)_{3}$ further to $\mathrm{SU}(2)_{3} \otimes \mathrm{U}(1)$, let us write the $\mathrm{SU}(3)_{3}$ irreps in the $\mathrm{SU}(2)_{3} \otimes \mathrm{U}(1)$ basis:

$$
\begin{gathered}
\mathbf{3}=\mathbf{2}(+1)+\mathbf{1}(-2), \\
\mathbf{8}=\mathbf{1}(0)+\mathbf{3}(0)+\mathbf{2}(+3)+\mathbf{2}(-3),
\end{gathered}
$$

where the $\mathrm{U}(1)$ charge is again given in the parentheses. Recall that the $\mathbf{8}$ of $\mathrm{SU}(3)_{3}$ in Eq. (4.25) has $J_{\mathrm{SO}(8) L}=0(\bmod 3)$. Under the $\mathbf{Z}_{6}$ twist, the $\mathbf{1}$ and the $\mathbf{3}$ have $J_{\mathrm{SO}(8) L}=0$, while the 2's have $J_{\mathrm{SO}(8) L}=3$. This property may be seen by going back to Eq. (4.24). So the final gauge symmetry becomes $\quad \mathrm{SU}(2)_{1} \otimes \mathrm{U}(1) \otimes \mathrm{SU}(2)_{3} \otimes \mathrm{U}(1) \otimes$ $\mathrm{SO}(10)_{3} \otimes \mathrm{U}(1)$. Recall that the $\mathbf{1 0}$ of $\mathrm{SU}(3)_{3}$ in Eq. (4.25) has $J_{\mathrm{SO}(8) L}=1(\bmod 3)$. Under $\mathrm{SU}(3)_{3} \supset \mathrm{SU}(2)_{3} \otimes \mathrm{U}(1)$,

$$
\mathbf{1 0}=\mathbf{1}(-6)+\mathbf{2}(-3)+\mathbf{3}(0)+\mathbf{4}(+3),
$$

where the $\mathbf{1}$ and the $\mathbf{3}$ have $J_{\mathrm{SO}(8) L}=4$, while the $\mathbf{2}$ and the 4 have $J_{\mathrm{SO}(8) L}=1$. So the constraint (4.22) cuts the three copies of $(\mathbf{1}, \mathbf{1 0}, \mathbf{1})(0)_{L}$ to one copy of the $\mathbf{1}$ and $\mathbf{3}$ (with $N^{1}= \pm 1$ ) and two copies of $\mathbf{2}$ and the $\mathbf{4}$ (with $\left.N^{2}+N^{3}= \pm 1\right)$. It also cuts the $(\mathbf{1 , 1 , 4 5})(0)$ from three copies to one copy. The resulting massless spectrum in the untwisted sector of the final model is summarized in the first column of Table I.

Now we turn to the twisted sectors; again, we consider the $A 1$ model first. In the twisted sectors $\overline{\alpha V}$, where $\alpha_{1}=1,2$, we note that the states in the $\alpha_{1}=2$ sectors are conjugates of the states in the corresponding $\alpha_{1}=1$ sectors. This allows us to concentrate on the $\alpha_{1}=1$ sectors. Also, states in the $\alpha_{0}=1$ sectors are the fermionic superpartners of the states in the $\alpha_{0}=0$ sectors. So we need to consider only the $\alpha_{0}=\alpha_{1}=1$ sector, where

$$
\overline{V_{0}+V_{1}}=\left[-\frac{1}{2}\left(\frac{1}{6} \frac{1}{3}\right)^{3}|| 0\left(\frac{1}{3}\right)^{2} \mid\left(\frac{1}{3}\right)^{5} 0_{r}^{5}\left(\frac{2}{3}\right)_{r}\right] .
$$

In the $A 1$ model, $\alpha^{*}=1$ and $t^{*}=t_{1}=m_{1}=3$. The invariant sublattice $I\left(\overline{V_{0}+V_{1}}\right)$ is given by $\Gamma^{2} \otimes \Gamma^{6}$, where $\Gamma^{2}$ is the $\mathrm{SU}(3) \quad$ lattice and $\Gamma^{6}=\{(\sqrt{3} \mathbf{q} \mid Q)\}$, where $(\mathbf{q} \mid Q)$ $=(\mathbf{0} \mid 0),(\mathbf{v} \mid V),(\mathbf{s} \mid S),(\mathbf{s} \mid \bar{S})$. The dual lattice is $\widetilde{I}\left(\overline{V_{0}+V_{1}}\right)$ $=\widetilde{\Gamma}^{2} \otimes \widetilde{\Gamma}^{6}$, where $\widetilde{\Gamma}^{6}=\{(\mathbf{q} / \sqrt{3} \mid Q)\}$. The momenta in the twisted sector belong to the shifted dual lattices $\widetilde{\Gamma}^{2} \otimes \widetilde{\Gamma}_{s}^{6}$ where $\widetilde{\Gamma}_{s}^{6}=\widetilde{\Gamma}^{6}+(\mathbf{0} \mid 2 / 3)$.

The determinant of the metric of the invariant sublattice is $M(\overline{\alpha V})=3^{6}$, and the number of fixed points is given by

$$
\xi(\overline{\alpha V})=[2 \sin (\pi / 3)]^{10}[M(\overline{\alpha V})]^{-1 / 2}=9 .
$$

We see that the only nontrivial contribution to the number of fixed points in the twisted sector comes from the symmetric $\mathbf{Z}_{3}$ twist in $\Gamma^{4,4}$. This twist contributes $9=3_{R} \times 3_{L}$ fixed points; i.e., they come from both left- and right-moving world-sheet conformal fields corresponding to irreps $\mathbf{3}_{L} \otimes \mathbf{3}_{R}$ of the underlying current subalgebra $\mathrm{SU}(3)_{L} \otimes \mathrm{SU}(3)_{R}$. In this $\overline{V_{0}+V_{1}}$ twisted sector, the spectrum-generating formula reads

$$
\begin{gathered}
V_{0} \cdot \mathcal{N}=\frac{1}{2}\left(N^{0}+N^{1}+N^{2}+N^{3}\right)=\frac{1}{2}(\bmod 1), \\
V_{1} \cdot \mathcal{N}+f_{i}\left(\overline{\alpha V}, n_{1}\right)+\frac{1}{2} \vec{Q}^{2}(\vec{P} \overline{\alpha V})=0(\bmod 1),
\end{gathered}
$$

where $n_{1}=0,1,2$. Knowing $\xi(\overline{\alpha V})=9$, it follows from Eqs. (2.10), (2.16), and (2.17) that $\xi\left(\overline{\alpha V}, n_{1}\right)=9$ for $n_{1}=0$, and zero otherwise. So we need consider only the $n_{1}=0$ term in Eq. (4.31). Recall that $\vec{P} \overline{\alpha V} \in \widetilde{I}(\overline{\alpha V})$; also, $\vec{Q}(\vec{P} \overline{\alpha V}) \in \widetilde{I}(\overline{\alpha V})$ is an arbitrary vector such that $\vec{P} \overline{\alpha V}-\alpha^{*} \vec{Q}(\vec{P} \overline{\alpha V}) \in I(\overline{\alpha V})$. For $\alpha_{1}=1$ we can choose $\vec{Q}(\vec{P} \overline{\alpha V})=\vec{P} \overline{\alpha V}$. Again, let us consider only the massless states. Since $E^{R}=0$ already, we can ignore the right-moving $\left(N^{1}, N^{2}, N^{3}\right)$ and $J^{\ell}$ in Eqs. (4.30) and (4.31). So Eq. (4.30) implies $N^{0}=1$, i.e., antiparticle states. Since all the SU(3) nontrivial states in $\Gamma^{2,2}$ are massive, we may also ignore them. The resulting momenta $\mathbf{p}$ in this twisted sector are in $\widetilde{\Gamma}_{s}^{6}$ i.e., $\mathbf{p}=\left(\mathbf{q} / \sqrt{3} \mid \frac{2}{3}+Q\right)$. Now the left-moving energy is given by

$$
E^{L}=-\frac{2}{9}+\frac{1}{2}\left(\frac{2}{3}+Q\right)^{2}+\frac{1}{6} \mathbf{q}^{2}
$$

and the constraint (4.31) reduces to

$$
\frac{2}{3} Q+\frac{1}{2} \vec{P}_{L}^{2}=0(\bmod 1),
$$

where $\vec{P}_{L}=(\mathbf{q} / \sqrt{3} \mid Q)$. Here $J_{\mathrm{SO}(8) L}=J_{\mathrm{SO}(10)}=0$, since their excitations are too heavy. Note that $\mathbf{p}=\vec{P}_{L}+\left(\mathbf{0} \mid \frac{2}{3}\right)$. The only possible massless states come from $\vec{P}_{L}=(\mathbf{0} \mid 0),(\mathbf{v} / \sqrt{3} \mid-1)$, and $(\mathbf{c} / \sqrt{3} \mid C)$. It is easy to check that these choices of $\vec{P}_{L}$ (or p) also satisfy the above spectrum-generating formulas; i.e., the level-matching condition [14] is sufficient. Now we can put them together with the $\alpha_{1}=2$ sector, resulting in, among other fields, nine right-handed antispinors of $\mathrm{SO}(10)$. We shall rewrite them as left-handed $\mathrm{SO}(10)$ spinors.

The hidden sector gauge group is $\mathrm{SU}(3)_{1}$, and no massless fields with its quantum numbers appear. The rightmoving fixed points correspond to the number of generations of chiral fermions, whereas the three left-moving fixed points appear in some irrep of the gauge group $\mathrm{SU}(3)_{3}$. That they form the 3 of $\mathrm{SU}(3)_{3}$ is easily seen in the alternative construction given in the next section. Here an examination of their scattering may be used to fix their quantum numbers. However, as we shall see, it is easiest to use the $\mathrm{SU}(3)_{3}$ anomaly-free condition to uniquely fix their quantum numbers. In summary, we have three $N=1$ chiral supermultiplets in $(\mathbf{1}, \mathbf{3}, \mathbf{1 6})(-1)_{L},(\mathbf{1}, \mathbf{3}, \mathbf{1 0})(+2)_{L}$, and $(\mathbf{1 , 3}, \mathbf{1})(-4)_{L}$, where the radius of the $\mathrm{U}(1)$ boson has been shifted from $1 / 2$ to $1 / 6$. This completes the massless spectrum of the $A 1$ model. Note that the $\mathrm{SU}(3)_{3}$ chiral anomaly in the twisted sectors is canceled by that in the untwisted sector as a $\mathbf{1 0}_{L}$ of $\mathrm{SU}(3)_{3}$ has 27 times the anomaly contribution of a $\boldsymbol{3}_{L}$. The model is also $\mathrm{U}(1)$ anomaly free due to the underlying $\mathrm{E}_{6}$ structure of the $\mathrm{SO}(10)_{3} \otimes \mathrm{U}(1)$ matter fields as can be seen 
from the branching $\mathbf{2 7}=\mathbf{1 6}(-1)+\mathbf{1 0}(+2)+\mathbf{1}(-4)$ under $\mathrm{E}_{6} \supset \mathrm{SO}(10) \otimes \mathrm{U}(1)$. Here, $\mathrm{SU}(3)_{3} \otimes \mathrm{U}(1)$ may be interpreted as the horizontal symmetry.

Now we return to the final model. The $\mathbf{Z}_{2}$-invariant states in the above twisted sector form the $\mathbf{Z}_{3}$-twisted sector of the final model, namely, the $T 3$ sector (i.e., $\alpha^{*}=2,4$, with $\left.t^{*}=6\right)$. Under the $Z_{6}$ twist, we have additional twisted sectors, namely, $T 6$ (i.e., $\alpha^{*}=1,5$ ) and $T 2$ (i.e., $\alpha^{*}=3$ ). The number of fixed points $\xi(\overline{\alpha V}, \mathbf{n})$ for each twisted sector and for each $\mathbf{n}$ is given as

$\begin{array}{ccccccc}\mathbf{n}=\left(n_{1}, n_{2}\right) & (0,0) & (0,1) & (1,0) & (1,1) & (2,0) & (2,1) \\ T 6 & 1 & 0 & 0 & 0 & 0 & 0 \\ T 3 & 5 & 4 & 0 & 0 & 0 & 0 \\ T 2 & 2(6) & 0 & 1(5) & 0 & 1(5) & 0\end{array}$

Here the number of fixed points in the $T 2$ sector before the inclusion of the invariant lattice volume factor is given in parentheses. It is easy to see that the $V_{1}$ constraint changes nothing in the spectrum, as expected. So all we need is to impose the $V_{2}$ constraint on the $\overline{V_{0}+V_{1}}$ sector:

$$
V_{2} \cdot \mathcal{N}+f_{2}\left(\overline{V_{0}+V_{1}}, \mathbf{n}\right)+3 \vec{Q}^{2}\left(\vec{P} \overline{V_{0}+V_{1}}\right)=0(\bmod 1),
$$

which reduces to the constraint $f_{2}\left(\overline{V_{0}+V_{1}}, \mathbf{n}\right)=0$, which is satisfied only for $\mathbf{n}=(0,0)$. From the above table, we see that this gives $\xi\left(\overline{V_{0}+V_{1}}, \mathbf{n}=(0,0)\right)=5$ fixed points. Actually, this result is easily seen in the standard orbifold construction. Of the original nine fixed points, the one at the origin is invariant under the $\mathbf{Z}_{2}$ twist. The remaining eight fixed points form four pairs, and the $\mathbf{Z}_{2}$ twist permutes the two fixed points in each pair. Forming four symmetric and four antisymmetric combinations, we have $9=5(1)+4(-1)$ (where the $\mathbf{Z}_{2}$ phases are given in parentheses); that is, five of the original nine are invariant under the $\mathbf{Z}_{2}$ twist. So these five copies of the $\mathrm{SO}(10)_{3}$ chiral matter fields survive, while the other four are projected out. They transform in the irreps of $\mathrm{SU}(2)_{3} \otimes \mathrm{U}(1)$. We can see this result from yet another viewpoint. We may treat the nine fixed points as $9=3_{R} \times 3_{L}$. The $\mathbf{Z}_{2}$ phases of these fixed points can be understood in terms of the branching $3_{R, L}=1_{R, L}(1)$ $+2_{R, L}(-1)$ (the $\mathbf{Z}_{2}$ phases are given in parentheses). Therefore, we have $9=5(1)+4(-1)$ (here $5=2_{R} \times 2_{L}+1_{R} \times 1_{L}$ and $\left.4=2_{R} \times 1_{L}+1_{R} \times 2_{L}\right)$. So we end up with two copies of $(\mathbf{1 , 2}, \mathbf{1 6})(0,-1,-1)_{L}$ and one copy of $(\mathbf{1}, \mathbf{1}, \mathbf{1 6})$ $\times(0,+2,-1)_{L}$, plus the corresponding vector and singlet irreps of $\mathrm{SO}(10)_{3}$ [the $\mathrm{U}(1)$ charges are normalized to $(1 / \sqrt{6}, 1 / 3 \sqrt{6}, 1 / 6)]$.

In the final model, we have other twisted sectors besides the $T 3$ sector. Let the $V_{2}$ and $V_{0}+V_{2}$ sectors form the $T 2$ sector, while the $V_{1}+V_{2}, V_{0}+V_{1}+V_{2}, 2 V_{1}+V_{2}$, and $V_{0}+2 V_{1}+V_{2}$ sectors form the $T 6$ sector. Let us first consider the $V_{0}+V_{1}+V_{2}$ sector:

$$
\overline{V_{0}+V_{1}+V_{2}}=\left(-\frac{1}{2}\left(\frac{1}{6} \frac{1}{3}\right)\left(-\frac{1}{3} \frac{5}{6}\right)^{2}|| \frac{e_{1}}{2}\left(\frac{5}{6}\right)^{2} \mid\left(\frac{1}{3}\right)^{5} 0_{r}^{5}\left(\frac{2}{3}\right)_{r}\right) .
$$

The sublattice invariant under the $\mathbf{Z}_{6}$ twist is the same as that for the $\mathbf{Z}_{3}$ twist, $I\left(\overline{V_{0}+V_{1}+V_{2}}\right)=\Gamma^{2} \otimes \Gamma^{6}$. So the number of fixed points in the $T 6$ sector is one. From the table above, $\xi\left(\overline{V_{0}+V_{1}+V_{2}}, \mathbf{n}\right)=1$ for $\mathbf{n}=(0,0)$ only, and zero, otherwise. Again, since the ground state has $E^{R}=0$ already, no right-moving excitation is allowed for massless states; so we may ignore all right-moving quantum numbers except $N^{0}$. The left-moving energy is

$$
E^{L}=-\frac{11}{36}+\frac{1}{2}\left(e_{1}+\widetilde{p}_{L}\right)^{2}+\frac{1}{2} \widetilde{P}_{\mathrm{SO}(10)}^{2}+\frac{1}{2}\left(\frac{e_{1}}{2}+Q\right)^{2}+\cdots,
$$

where contributions that yield only massive states are ignored. Here $\left(\widetilde{p}_{L}\left|\widetilde{P}_{\mathrm{SO}(10)}\right| Q\right)$ is in the dual invariant lattice $\widetilde{I}\left(\overline{V_{0}+V_{1}+V_{2}}\right)=\widetilde{\Gamma}^{2} \otimes \widetilde{\Gamma}^{6}$, where $\widetilde{\Gamma}^{6}=\{(\mathbf{q} / \sqrt{3} \mid Q)\}$. The only possible massless states in this twisted sector come from the shifted momenta $\left(\frac{1}{2} e_{1}+\widetilde{p_{L}}|\mathbf{q} / \sqrt{3}| \frac{2}{3}+Q\right)$, where $\widetilde{p_{L}}=-\widetilde{e}^{-1}$ and $\left(-e_{1}+\widetilde{e}^{-1}\right)$, while $(\mathbf{q} / \sqrt{3} \mid Q)=(\mathbf{0} \mid 0),(\mathbf{v} / \sqrt{3} \mid-1)$ and (c/ $\sqrt{3} \mid-1 / 2)$. It is straightforward to check that they all satisfy the corresponding spectrum-generating formulas for this sector, with $N^{0}=0$. Here $\frac{1}{2} e_{1}+\widetilde{p_{L}}$ corresponds to $\mathrm{U}(1)$ charges, and so they are singlets under $\mathrm{SU}(2)_{1} \otimes \mathrm{SU}(2)_{3}$. Together with the $\overline{V_{0}+2 V_{1}+V_{2}}$ sector, the massless chiral fields are $(\mathbf{1}, \mathbf{1}, \mathbf{1 6})( \pm 1,-1,-1)_{R}$, plus the corresponding vector and singlet irreps of $\mathrm{SO}(10)_{3}$, where the radius of the first $U(1)$ is $1 / \sqrt{6}$. Note that these states are right handed, and come in pairs [ \pm 1 of the first U(1) charge]. So, effectively, we have a total of $3=5-2$ left-handed chiral families of 16's.

Finally, let us consider the space-time fermions in the $T 2$ sector,

$$
\bar{V}_{0}+V_{2}=\left(-\frac{1}{2}\left(-\frac{1}{2} 0\right)\left(0 \frac{1}{2}\right)^{2}|| \frac{e_{1}}{2}\left(\frac{1}{2}\right)^{2} \mid 0^{5} 0_{r}^{5} 0_{r}\right)
$$

and $W\left(\overline{V_{0}+V_{2}}\right)=W_{2}$. It is convenient to first impose the $V_{0}$ and $V_{2}$ constraints in the spectrum-generating formula, and then project onto $\mathbf{Z}_{3}$ invariant-states (i.e., impose the $V_{3}$ constraint). This is equivalent to considering the twisted 
sector of the $A 2$ model or the $\mathbf{Z}_{2}$ orbifold of the $N 1$ model. The sublattice invariant under the $\mathbf{Z}_{2}$ twist is given by the sublattice of $\Gamma^{2,2} \otimes \Gamma^{16}$ invariant under the Wilson lines $U_{1}$ and $U_{2}$. The metric of this sublattice has determinant 16 . Therefore, the number of fixed points is $4_{R} \times 4_{L} / \sqrt{16}$ $=2_{R} \times 2_{L} . \quad$ The $\mathbf{Z}_{2}$ orbifold breaks $\mathrm{SU}(3) \otimes \mathrm{SO}(8)$ to $\mathrm{SU}(2)_{1} \otimes \mathrm{U}(1) \otimes \mathrm{SU}(2)^{4}$. The $2_{L}$ fixed points form a doublet of one of the $\mathrm{SU}(2)$ factor in $\mathrm{SU}(2)^{4}$, say, the last $\mathrm{SU}(2)$. Again the ground state $E^{R}=0$, while the left-moving energy is

$$
E^{L}=-\frac{3}{4}+\frac{1}{2}\left(\frac{e_{1}}{2}+p_{L}\right)^{2}+\frac{1}{2}\left(n_{1}^{5}+\vec{n}_{1}^{5}+n_{1}^{6}+{ }_{1}^{6}\right)+\cdots,
$$

where only terms that can contribute to the massless spectrum are included. [Note that all the states in nontrivial irreps of $\mathrm{SO}(10)_{3} \otimes \mathrm{U}(1)$ are massive.] At $E^{L}=-\frac{1}{2}$, we have two copies of (off-shell) $(\mathbf{2}, \mathbf{1}, \mathbf{1}, \mathbf{1}, \mathbf{2})(0)$. They come from $p_{L}=0$ and $p_{L}=-e_{1}$, with shifted momenta $e_{1} / 2+p_{L}$ $= \pm e_{1} / 2$, forming a doublet of $\mathrm{SU}(2)_{1}$. To obtain the physical massless states, we need $E^{L}=0$. There are two types of such states: (i) We can excite one of the four oscillator modes indicated in Eq. (4.39), yielding eight massless states, and (ii) we can change the momentum to $p_{L}=e_{2}$ and $p_{L}=-e_{1}-e_{2}$; since the shifted momenta $\pm\left(e_{2}+e_{1} / 2\right)$ are now orthogonal to the shift $e_{1} / 2$, they are $\mathrm{SU}(2)_{1}$ singlets, and so they form a pair of U(1) charged states. For these massless states, we see that they all satisfy the $V_{0}$ and $V_{2}$ constraints

$$
\begin{gathered}
\frac{1}{2}\left(N^{0}+N^{1}\right)=\frac{1}{2}(\bmod 1), \\
\frac{e_{1}}{2} p_{L}-\frac{1}{2} J_{\mathrm{SO}(8) L}=\frac{1}{2}(\bmod 1) .
\end{gathered}
$$

Collecting these states, we have two massless sets of $(\mathbf{2}, \mathbf{2}, \mathbf{2}, \mathbf{2}, \mathbf{1})(0)$ and $(\mathbf{1}, \mathbf{1}, \mathbf{1}, \mathbf{1}, \mathbf{2})( \pm 3)$. Note that their quantum number assignments are uniquely fixed by the underlying $\mathbf{Z}_{3}$ symmetry [i.e., the cyclic permutation of the first three SU(2)'s in SU(2) $\left.{ }^{4}\right]$ and the counting of states. To obtain the states in the final model, consider the action of the $\mathbf{Z}_{3}$ twist, i.e., the $V_{1}$ constraint,

$$
f_{1}+\frac{1}{3}\left(N^{1}-J_{\mathrm{SO}(8) L}\right)=\frac{2}{3}(\bmod 1) .
$$

To reach the gauge symmetry of the final model, the $\mathbf{Z}_{3}$ twist breaks the last $\mathrm{SU}(2)$ to $\mathrm{U}(1)$ and converts the middle three $\mathrm{SU}(2)$ 's to $\mathrm{SU}(2)_{3}$. It also leaves the first $\mathrm{SU}(2)$ alone. Under the $\mathbf{Z}_{3}$ rotation, the fixed points have phases $2_{L, R}$ $=1_{L, R}(\omega)+1_{L, R}\left(\omega^{2}\right)$. The $(\mathbf{2 , 2 , 2})$ of $\mathrm{SU}(2)^{3}$ become $\mathbf{2 , 2}$, and 4 of $\mathrm{SU}(2)_{3}$. So the resulting $\mathbf{Z}_{3}$-invariant massless states are $(\mathbf{2}, \mathbf{2})_{L}$ and $(\mathbf{2}, \mathbf{4})_{L}\left[\right.$ in $\left.\mathrm{SU}(2)_{1} \otimes \mathrm{SU}(2)_{3}\right]$ plus a pair of singlets. To clarify the notation, here we point out that by the subscript $L$ in the case of fermions in a real irrep of a gauge group [say, $(\mathbf{2 , 2})_{L}$ of $\mathrm{SU}(2)_{1} \otimes \mathrm{SU}(2)_{3}$ ] we mean a two component field.

This concludes the construction of the three-family $\mathrm{SO}(10)$ grand unified model. The massless spectrum is summarized in the first column in Table I. There, the states are grouped into sectors, the untwisted sector $U\left(\alpha^{*}=0\right)$ and the twisted sectors $T 3\left(\alpha^{*}=2,4\right), T 6\left(\alpha^{*}=1,5\right)$, and $T 2$ $\left(\alpha^{*}=3\right)$. This grouping implements the selection rules for string couplings, since the sum of $\alpha^{*} \mathrm{~s}$ of all the incoming particles at each vertex must be zero $(\bmod 6)$. For example, a particle in the $T 2$ sector coupling to a particle in the $T 3$ sector can only go to a particle in the $T 6$ sector.

\section{ALTERNATIVE CONSTRUCTION}

In this section we will describe the model considered in the previous section in a different basis in which the quantum numbers of the states are more transparent. This is not surprising, since it is known that some twists can be reexpressed as shifts. Again, let us start with the $N 1$ Narain model (that was obtained in the previous section by starting with the $N 0$ model and turning on Wilson lines). This model has $N=4$ space-time supersymmetry and the gauge group $\mathrm{SU}(3) \otimes \mathrm{SO}(8) \otimes \mathrm{SO}(10)^{3} \otimes \mathrm{SO}(2)$. Recall that the internal momenta span an even self-dual Lorentzian lattice $\Gamma^{\prime 6,22}$ described in the previous section. Let us work in the basis where the right-moving momenta corresponding to the $\Gamma^{4,4}$ sublattice of the original lattice $\Gamma^{6,22}=\Gamma^{2,2} \otimes \Gamma^{4,4} \otimes \Gamma^{16}$ are described in terms of the branching $\mathrm{SO}(8) \supset \mathrm{SU}(3) \otimes \mathrm{U}(1)^{2}$, used in the previous section, whereas the left-moving $\mathrm{SO}(8)$ momenta are written in terms of the branching $\mathrm{SO}(8)$ $\supset \mathrm{SU}(2)^{4}$. The $\mathrm{SO}(8)$ weights $\mathbf{0}, \mathbf{v}, \mathbf{s}$, and $\mathbf{c}$ read:

$$
\begin{aligned}
& 1=(1,1,1,1)+(2,2,2,2), \\
& \mathbf{8}_{v}=(2,2,1,1)+(1,1,2,2), \\
& \mathbf{8}_{s}=(1,2,2,1)+(2,1,1,2), \\
& 8_{c}=(2,1,2,1)+(1,2,1,2) .
\end{aligned}
$$

Note that under cyclic permutations of the first three SU(2)'s the irreps $\mathbf{8}_{v}, \boldsymbol{8}_{s}$, and $\boldsymbol{8}_{c}$ are permuted. This is the same as one of the $\mathbf{Z}_{3}$ symmetries that we considered in the previous section, namely, the triality symmetry of the $\mathrm{SO}(8)$ Dynkin diagram. The second $\mathbf{Z}_{3}$ twist that we considered in the previous section rotates the bosons corresponding to the $\mathrm{SU}(3)$ subgroup by $2 \pi / 3$. In the $\mathrm{SU}(2)^{4}$ basis this twist is represented by a shift, namely, the $\mathbf{Z}_{3}$ shift $-\sqrt{2} / 3$ [note that $\sqrt{2}$ is the simple root of $\mathrm{SU}(2)]$. It is convenient to introduce new bosons corresponding to the first three SU(2)'s. Let $\eta_{1}, \eta_{2}, \eta_{3}$, and $\eta_{4}$ be the real bosons of $\mathrm{SU}(2)^{4}$. Next, let $\Sigma=\left(\eta_{1}+\omega^{2} \eta_{2}+\omega \eta_{3}\right) / \sqrt{3}$, and so its complex conjugate $\Sigma^{\dagger}=\left(\eta_{1}+\omega \eta_{2}+\omega^{2} \eta_{3}\right) / \sqrt{3} \quad[$ here $\omega=\exp (2 \pi i / 3)]$, and $\rho=\left(\eta_{1}+\eta_{2}+\eta_{3}\right) / \sqrt{3}$. In this basis, we have one complex boson $\Sigma$, and two real bosons $\rho$ and $\eta_{4}$. The outer automorphism of the first three $\mathrm{SU}(2)$ 's that permutes them is now a $\mathbf{Z}_{3}$ twist of $\Sigma\left(\Sigma^{\dagger}\right)$ that are eigenvectors with eigenvalues $\omega\left(\omega^{2}\right)$. In the meantime, $\rho$ and $\eta_{4}$ are invariant under this twist, and therefore unaffected. The other $\mathbf{Z}_{3}$ twist mentioned earlier corresponds to shifting the momentum lattice of $\eta_{4}$ by $-\sqrt{2} / 3$. This shift has no effect on $\rho$ and $\Sigma\left(\Sigma^{\dagger}\right)$.

Next let us consider the $\mathbf{Z}_{2}$ twist in this new basis. It breaks $\mathrm{SO}(8)$ to $\mathrm{SU}(2)^{4}$. In the $\mathrm{SU}(2)^{4}$ language this can be achieved by a $\mathbf{Z}_{2}$ shift $\sqrt{2} / 2$ in one of the SU(2)'s. For this shift to be compatible with the above $\mathbf{Z}_{3}$ twist, we must shift the last $\mathrm{SU}(2)$ boson $\eta_{4}$. As we shall see, this shift, combined with the above outer automorphism of the first three $\mathrm{SU}(2)$ 's and accompanied by a $\mathbf{Z}_{3}$ shift in the last $\mathrm{SU}(2)$ as described above, exactly corresponds to the $\mathbf{Z}_{6}$ orbifold of 
$\Gamma^{4,4}$ considered in the previous section (provided that the right movers are twisted as before).

Thus, in this new basis, the set of vectors acting on the $N 1$ model considered in the previous section is given by

$$
\begin{gathered}
V_{0}=\left[-\frac{1}{2}\left(-\frac{1}{2} 0\right)^{3}|| 0\left|00_{r} 0_{r}\right| 0^{5} 0_{r}^{5} 0_{r}\right], \\
V_{1}=\left[0\left(-\frac{1}{3} \frac{1}{3}\right)^{3}|| 0\left|\left(\frac{2}{3}\right) 0_{r}\left(-\frac{\sqrt{2}}{3}\right)_{r}\right|\left(\frac{1}{3}\right)^{5} 0_{r}^{5}\left(\frac{2}{3}\right)_{r}\right], \\
V_{2}=\left[0(00)\left(-\frac{1}{2} \frac{1}{2}\right)^{2}|| \frac{e_{1}}{2}\left|00_{r}\left(\frac{\sqrt{2}}{2}\right){ }_{r}\right| 0^{5} 0_{r}^{5} 0_{r}\right], \\
W_{1}=\left[0\left(0 \frac{1}{3}\right)^{3}|| 0\left|\left(\frac{1}{2}\right) 0_{r} 0_{r}\right|\left(\frac{1}{2}\right)^{5} 0_{r}^{5} 0_{r}\right] \\
W_{2}=\left[0(00)\left(0 \frac{1}{2}\right)^{2}|| 0\left|00_{r} 0_{r}\right| 0^{5} 0_{r}^{5} 0_{r}\right] .
\end{gathered}
$$

Here we have separated the left-moving SU(3) complex boson, the first left-moving entry, from the left-moving $\mathrm{SU}(2)^{4}$ bosons by a single vertical line. The second leftmoving entry corresponds to the $\Sigma$ complex boson. The third and fourth entries are for $\rho$ and $\eta_{4}$, respectively. The rest of them are the same as in the previous section, and correspond to the $\mathrm{SO}(10)^{3} \otimes \mathrm{SO}(2)$ bosons. Note that we have deliberately chosen the monodromy of the $\mathbf{Z}_{3}$ twist that acts on the left-moving $\mathrm{SO}(8)$ bosons to be $2 / 3$; the other choice, $1 / 3$, gives an equivalent model, but only the former choice can be analyzed using the simplified rules of Sec. III; in this case, one can check that the condition (3.24) is satisfied.

First we consider the model generated by the vectors $\left\{V_{0}, V_{1}\right\}$. Let us first consider the untwisted sector of this model. The gauge bosons come in the adjoint of $\mathrm{SU}(3)_{1} \otimes \mathrm{SU}(3)_{3} \otimes \mathrm{SO}(10)_{3} \otimes \mathrm{U}(1)$. The appearance of the $\mathrm{SU}(3)_{1} \otimes \mathrm{SO}(10)_{3} \otimes \mathrm{U}(1)$ gauge bosons should be clear from the previous section. Let us concentrate on the $\mathrm{SU}(3)_{3}$ gauge bosons. Note that the $\mathrm{SU}(2)^{4}$ subgroup of $\mathrm{SO}(8)$ breaks to $\mathrm{SU}(2)_{3} \otimes \mathrm{U}(1)$ as a result of the outer automorphism twist performed on the first three SU(2)'s and the shift in the momentum lattice of the last $\mathrm{SU}(2)$ which breaks it to $\mathrm{U}(1)$. However, there are additional gauge bosons that come from the original $\mathrm{SO}(8)$ gauge bosons which in the $\mathrm{SU}(2)^{4}$ basis read $\left(\mathbf{2 , 2 , 2 , 2 )}\right.$. Under the $\mathbf{Z}_{3}$ twist that permutes the first three $\mathrm{SU}(2)$ 's we have, in the branching $\mathrm{SU}(2)^{3} \supset \mathrm{SU}(2)_{3}$,

$$
(\mathbf{2 , 2}, \mathbf{2})=\mathbf{4}(1)+\mathbf{2}(\omega)+\mathbf{2}\left(\omega^{2}\right),
$$

where the $\mathbf{Z}_{3}$ phases are given in parentheses. Introducing $J_{\mathrm{SU}(2)}$ for the first three $\mathrm{SU}(2)$ 's, this means $J_{\mathrm{SU}(2)}=0,1,2$ for the above three irreps, respectively. Under the $\mathbf{Z}_{3}$ shift that breaks the fourth $\mathrm{SU}(2)$ to $\mathrm{U}(1)$, we have $\mathbf{2}=(+3)+(-3)$, and $\mathbf{3}=(0)+(+6)+(-6)$, where the normalization of this $\mathrm{U}(1)$ charge is $1 / 3 \sqrt{2}$. Now, Eq. (4.25) can be rewritten in the $\mathrm{SU}(2)_{3} \otimes \mathrm{U}(1)$ basis,

$$
\begin{aligned}
\mathbf{2 8}= & \mathbf{8}+\mathbf{1 0}+\overline{\mathbf{1 0}} \\
= & {[\mathbf{1}(0)(0)+\mathbf{3}(0)(0)+\mathbf{2}(3)(2)+\mathbf{2}(-3)(1)] } \\
& +[\mathbf{1}(-6)(0)+\mathbf{3}(0)(1)+\mathbf{2}(-3)(2)+\mathbf{4}(3)(0)] \\
& +[\mathbf{1}(6)(0)+\mathbf{3}(0)(2)+\mathbf{2}(3)(1)+\mathbf{4}(-3)(0)],
\end{aligned}
$$

where the first set of parentheses gives the $\mathrm{U}(1)$ charge and the second set gives the $J_{\mathrm{SU}(2)}$ value. The spectrumgenerating formula reads, for both the $\mathbf{0}$ and the $V_{0}$ sectors (with $k_{00}=0$ for definiteness),

$$
\begin{aligned}
& V_{0} \cdot \mathcal{N}=\frac{1}{2} \sum_{r=0}^{3} N^{r}=\frac{1}{2}(\bmod 1) \\
& V_{1} \cdot \mathcal{N}= \frac{1}{3}\left(\sum_{r=1}^{3} N^{r}+\sum_{\ell=1}^{3} J^{\ell}-J_{\mathrm{SU}(2)}-J_{\mathrm{SO}(10)}\right. \\
&\left.+2 Q-\sqrt{2} p\left(\eta_{4}\right)\right) \\
&= 0(\bmod 1)
\end{aligned}
$$

Here $p\left(\eta_{4}\right)$ is the momentum of the $\eta_{4}$ real boson, and the above $\mathrm{U}(1)$ charge is equal to $3 \sqrt{2} p\left(\eta_{4}\right)$. For gauge bosons in the $\mathbf{0}$ sector, $N^{0}= \pm 1$. Since $\Sigma_{\ell=1}^{3} J^{\ell}=J_{\mathrm{SO}(10)}=0$ for massless states, the above formula reduces to

$$
-\frac{1}{3}\left(\sqrt{2} p\left(\eta_{4}\right)+J_{\mathrm{SU}(2)}\right)=0(\bmod 1) .
$$

So among the $\mathbf{2 8}$ of $\mathrm{SO}(8)$, only the $2(3)(2)+2(-3)(1)$ plus the $\mathrm{SU}(2)_{3} \otimes \mathrm{U}(1)$ gauge bosons satisfy this constraint. From Eq. (4.26), we see that the resulting gauge group is $\mathrm{SU}(3)_{3}$. It is also easy to see that we have three copies of the Higgs fields in the adjoint of $\mathrm{SO}(10)_{3}$.

Although there are no massless states in the irreps of $\mathrm{SU}(3)_{1}$, there are, however, chiral fermions in the irrep of $\mathrm{SU}(3)_{3}$. Let us consider these states in the $V_{0}$ sector. Consider the left-handed states (i.e., the states with the lefthanded space-time chirality) which have $N^{0}=0$. Then there are three massless state solutions to $\sum_{r=1}{ }^{3} N^{r}=1(\bmod 2)$, namely, $\left(N^{1}, N^{2}, N^{3}\right)=(1,0,0),(0,1,0),(0,0,1)$; thus, there are three copies of the states we are discussing. The above constraint becomes

$$
\frac{1}{3}\left(1-\sqrt{2} p\left(\eta_{4}\right)-J_{\mathrm{SU}(2)}\right)=0(\bmod 1) .
$$

Of the states in Eq. (5.4), we see that only the decuplet $\mathbf{1 0}$ of $\mathrm{SU}(3)_{3}$ survives. Thus, in the untwisted sector we have three families of left-handed 10's of $\mathrm{SU}(3)_{3}$.

Next we turn to discussing the twisted sectors. Let us concentrate on the $\overline{V_{0}+V_{1}}$ twisted sector. (The $\overline{V_{0}+2 V_{1}}$ sector gives the conjugates of the states appearing in $\overline{V_{0}+V_{1}}$.) With our choice of $k_{00}=0$, the states in this sector are right handed. The sublattice $I\left(V_{1}\right) \subset \Gamma^{\prime 6,22}$ invariant under the twist part of $V_{1}$ is given by

$$
I\left(V_{1}\right)=\Gamma^{2} \otimes \Delta \otimes \Gamma^{6} .
$$

Here $\Gamma^{2}$ and $\Gamma^{6}$ are the same as in the previous section, and $\Delta=\left\{\left(\sqrt{\frac{3}{2}} n, \sqrt{\frac{1}{2}} n\right), n \in \mathbf{Z}\right\}$. The determinant of the metric of this lattice is $M\left(V_{1}\right)=3 \times 3 \times 3^{5}=3^{7}$, where the three factors come from the $\Gamma^{2}, \Delta^{2}$, and $\Gamma^{6}$ sublattices, respectively. Thus, the number of fixed points in the twisted sector $\overline{V_{0}+V_{1}}$ is given by

$$
\xi\left(\overline{V_{0}+V_{1}}\right)=[2 \sin (\pi / 3)]^{9} / \sqrt{M\left(V_{1}\right)}=3 .
$$


The momenta in the $\overline{V_{0}+V_{1}}$ sector belong to the shifted lattice $\widetilde{I}\left(V_{1}\right)+(\mathbf{0})(0 \mid \sqrt{2} / 3)(\mathbf{0} \mid 2 / 3)$. Here $\widetilde{I}\left(V_{1}\right)=\widetilde{\Gamma}^{2} \otimes \widetilde{\Delta} \otimes \widetilde{\Gamma}^{6}$ is the lattice dual to $I\left(V_{1}\right) . \widetilde{\Gamma}^{2}$ and $\widetilde{\Gamma}^{6}$ are the same as in the previous section, and $\widetilde{\Delta}=\{(n / \sqrt{6}, n / \sqrt{2}), n \in \mathbf{Z}\}$. As in the previous section, we have states in the irreps of $\mathrm{SO}(10)_{3}$ $\otimes \mathrm{U}(1)$, namely, $\overline{\mathbf{1 6}}(+1), \mathbf{1 0}(-2)$, and $\mathbf{1}(+4)$ [with the $\mathrm{U}(1)$ charge radius 1/6]. These states come in three copies (due to the three right-moving fixed points), and they also have $\mathrm{SU}(3)_{3}$ quantum numbers. For the states to be massless, the $\mathrm{SU}(2)_{3} \otimes \mathrm{U}(1)\left[\subset \mathrm{SU}(3)_{3}\right]$ quantum numbers must be $1\left[-2\right.$ ] [this state has $p\left(\eta_{4}\right)=p(\rho)=0$, where $p(\rho)$ is the momentum of the $\rho$ boson] and $\mathbf{2}[+1]$ [these states have $p\left(\eta_{4}\right)=1 / \sqrt{2}$ and $\left.p(\rho)= \pm 1 / \sqrt{6}\right]$. These states combine into 3 of SU(3) ${ }_{3}$. Thus, in the twisted sectors we have three families of left-handed $(\overline{\mathbf{3}}, \mathbf{1 6})(-1),(\overline{\mathbf{3}}, \mathbf{1 0})(+2)$, and $(\overline{\mathbf{3}}, \mathbf{1})(-4)$ [irreps of $\left.\mathrm{SU}(3)_{3} \otimes \mathrm{SO}(10)_{3} \otimes \mathrm{U}(1)\right]$. This concludes the discussion of the $\left\{V_{0}, V_{1}\right\}$ model, which is the same as the $A 1$ model constructed in the previous section.

Next we turn to the final model generated by the vectors $\left\{V_{0}, V_{1}, V_{2}\right\}$ Eq. (5.2). As in the previous section, to preserve $N=1$ space-time supersymmetry we must choose $k_{20}=1 / 2$. Then in the untwisted sectors, we simply keep the $\mathbf{Z}_{2}$-invariant states in the untwisted sector in the $A 1$ model. Since the $V_{2}$ vector breaks the original $\mathrm{SU}(3) \otimes \mathrm{SO}(8)$ gauge group to $\mathrm{SU}(2) \otimes \mathrm{U}(1) \otimes \mathrm{SU}(2)^{4}$, the gauge group in the final model $\quad$ is $\quad \mathrm{SU}(2) \otimes \mathrm{U}(1) \otimes \mathrm{SU}(2)_{3} \otimes \mathrm{U}(1) \otimes \mathrm{SO}(10)_{3} \otimes \mathrm{U}(1)$. Out of the three copies of matter fields in the untwisted sectors of the $\left\{V_{0}, V_{1}\right\}$ model, two copies have the $\mathbf{Z}_{2}$ phase -1 , while the other copy has the $\mathbf{Z}_{2}$ phase +1 . The singlet and the triplet of $\mathrm{SU}(2)_{3}$ in the branching (4.27) have the $\mathbf{Z}_{2}$ phase +1 , whereas the doublet and the quartet have the $\mathbf{Z}_{2}$ phase -1 . Therefore, we have one copy of the singlet and the triplet, and two copies of the doublet and the quartet [with the corresponding U(1) charges]. Also, only one copy of the $\mathrm{SO}(10)_{3}$ adjoint Higgs field survives.

Now consider the $\overline{V_{0}+V_{1}}$ twisted sector. There are two sources of $\mathbf{Z}_{2}$ phases. One is the fixed points, and the other one is the $\mathbf{3}$ of $\mathrm{SU}(3)_{3}$. In the branching $\mathbf{3}=\mathbf{1}(-2)$ $+2(+1)$ [under the breaking $\mathrm{SU}(3)_{3} \supset \mathrm{SU}(2)_{3} \otimes \mathrm{U}(1)$ ] the singlet has the $\mathbf{Z}_{2}$ phase +1 [since $p\left(\eta_{4}\right) / \sqrt{2}=0$ for this state], whereas the doublet has the $\mathbf{Z}_{2}$ phase -1 [since $p\left(\eta_{4}\right) / \sqrt{2}=1 / 2$ for these states]. Similarly, one of the fixed points has the $\mathbf{Z}_{2}$ phase +1 , while the other two have the $\mathbf{Z}_{2}$ phase -1 . This means that, say, out of the three copies of the left-handed states $(\overline{\mathbf{3}}, \mathbf{1 6})$ in the $T 3$ sector of the $\left\{V_{0}, V_{1}\right\}$ model, two copies of $(\mathbf{2}, \mathbf{1 6})_{L}$ and one copy of $(\mathbf{1}, \mathbf{1 6})_{L}$ survive the $\mathbf{Z}_{2}$ projection. That is, the $T 3$ sector gives rise to five left-handed spinors of $\mathrm{SO}(10)_{3}$ [as well as their vector and singlet counterparts with the corresponding $\mathrm{U}(1)$ charges]. The above discussion can be seen directly from the rules of Sec. III. The $V_{2}$ constraint in the $V_{1}$ sector becomes

$$
V_{2} \cdot \mathcal{N}_{V_{1}}+f_{2}\left(V_{1}, \mathbf{n}\right)=1 / 2(\bmod 1) .
$$

Now, $\xi\left(V_{1},\left(n_{1}, n_{2}\right)=(0,0)\right)=2, \xi\left(V_{1},\left(n_{1}, n_{2}\right)=(0,1)\right)=1$, and all the others are zero. Their fixed point phases are
$f_{2}\left(V_{1},\left(n_{1}, n_{2}\right)=(0,0)\right)=0$ and $f_{2}\left(V_{1},\left(n_{1}, n_{2}\right)=(0,1)\right)=1 / 2$, respectively. In the above discussion, the $\mathbf{Z}_{2}$ phases of the fixed points were defined as those that add up with the $V_{2} \cdot \mathcal{N}_{V_{1}}$ phases [which are the phases coming from the $\mathbf{3}$ of $\left.\mathrm{SU}(3)_{3}\right]$ to give zero. That is, the fixed point phases in our notation are given by $f_{2}\left(V_{1}, \mathbf{n}\right)-1 / 2$, so that two fixed points have the $\mathbf{Z}_{2}$ phase -1 and one 1 fixed point has the $\mathbf{Z}_{2}$ phase +1 , as inferred earlier. So the above spectrumgenerating formula gives us five generations of the $\mathrm{SO}(10)_{3}$ chiral matter fields.

Next consider the $T 6$ sector. The sublattice invariant under the twist part of $V_{1}+V_{2}\left(\mathbf{Z}_{6}\right.$ twist) is the same as $I\left(V_{1}\right)$. In these sectors the number of fixed points, therefore, can be seen to be 1 . Let us consider the $V_{0}+V_{1}+V_{2}$ twisted sector for definiteness. The states in this sector are left handed, as can be seen from the spectrum-generating formula. The number of massless chiral $\overline{\mathbf{1 6}}$ 's coming from this sector is 2 . These have opposite $\mathrm{U}(1)$ charges \pm 1 (with all the other quantum numbers the same), where this $U(1)$ is the one that comes from the $\mathrm{SU}(3)_{1} \supset \mathrm{SU}(2)_{1} \otimes \mathrm{U}(1)$ breaking (and is normalized to $1 / \sqrt{6}$ ). This means that the $T 6$ sector gives rise to two right-handed families of $\mathrm{SO}(10)_{3}$ [as well as their vector and singlet counterparts with the corresponding $\mathrm{U}(1)$ charges]. The net number of generations from the $T 3$ and $T 6$ sectors is, therefore, $5-2=3$.

Finally, let us discuss the $T 2$ sector. The invariant sublattice $I\left(V_{2}\right)=\Gamma^{2,18} \otimes \Gamma^{4}$. Here $\Gamma^{2,18}$ is an even self-dual Lorentzian lattice which can be obtained from $\Gamma^{2,2} \otimes \Gamma^{16}$ by turning on the Wilson lines similar to those that we use to construct $\Gamma^{\prime 6,22}$ from $\Gamma^{6,22}$ but without the entries corresponding to the $\Gamma^{4,4}$ sublattice of $\Gamma^{6,22}$. Recall that $\Gamma^{4}$ is the root lattice of $\mathrm{SO}(8)$, and the determinant of its metric is 4 . Therefore, the number of fixed points in the $T 2$ sector is $\xi\left(V_{2}\right)=[2 \sin (\pi / 2)]^{2} / \sqrt{4}=2$. One of these fixed points has the $\mathbf{Z}_{3}$ phase $\omega$, and the other one has the $\mathbf{Z}_{3}$ phase $\omega^{2}$. The momenta in the $T 2$ sector belong to the shifted lattice $\widetilde{I}\left(V_{2}\right)+\left(\mathbf{0} \| e_{1} / 2 \mid \mathbf{0 , 0}, \mathbf{0}, 0\right)(0,0,0,1 / \sqrt{2})$. [Here the first factor is a shift in the $\Gamma^{2,18}$ lattice, while the second factor, which is written in the $\mathrm{SU}(2)^{4}$ basis, is the shift in the $\widetilde{\Gamma}^{4}$ lattice.] Note that all the states with the $\mathrm{SO}(10)_{3} \otimes \mathrm{SO}(2)$ quantum numbers are massive. The only massless states come from the irreps of $\mathrm{SU}(2) \otimes \mathrm{U}(1) \otimes \mathrm{SU}(2)^{4}$ [with the $\mathrm{U}(1)$ radius $1 / \sqrt{6}]:(\mathbf{1} \mid \mathbf{1}, \mathbf{1}, \mathbf{1}, \mathbf{2})( \pm 3)$ and $(\mathbf{2} \mid \mathbf{2}, \mathbf{2}, \mathbf{2}, \mathbf{1})(0)$. After the $\mathbf{Z}_{3}$ projection we have [in the $\mathrm{SU}(2)_{1} \otimes \mathrm{SU}(2)_{3}$ irreps] one $(\mathbf{2 , 2})$, one $(\mathbf{2 , 4})$, and a singlet (see the first column in Table I). This completes our analysis of the alternative construction of the model.

\section{MODULI SPACE}

In Sec. IV we could start from the most general even self-dual Lorentzian lattice $\Gamma^{4,4}$ that admits a $\mathbf{Z}_{6} \otimes \mathbf{Z}_{6}$ symmetry so that both complex bosons can be simultaneously twisted. [Then the particular choice of the lattice with enhanced $\mathrm{SO}(8)$ gauge symmetry corresponds to a special point in the moduli space of such lattices.] Since the $\mathbf{Z}_{2} \otimes \mathbf{Z}_{2}$ symmetry is automatic, we need only to consider the $\mathbf{Z}_{3} \otimes \mathbf{Z}_{3}$ symmetry. The most general lattice that possesses such a symmetry can be written in the following $E_{I}, I=1,2,3,4$, basis, which proves to be useful for our purposes. Let us also define $\widetilde{E}^{I}$, so that $E_{I} \cdot \widetilde{E}^{J}=\delta_{I}^{J}$. Now, $\Gamma^{4,4}=\left\{\left(P_{R}|| P_{L}\right)\right\}$ with 


$$
P_{L, R}=\widetilde{E}^{I}\left(M_{I}-2 B_{I J} N^{J}\right) \pm E_{I} N^{I} / 2,
$$

where $M_{I}, N^{I} \in \mathbf{Z}, I=1,2,3,4 ; B_{I J}$ and $G_{I J}=E_{I} \cdot E_{J}$ are the antisymmetric and the metric constant background fields, respectively. Recall that $\widetilde{G}^{I J}=\widetilde{E}^{J} \cdot \widetilde{E}^{J}$ is the inverse of $G_{I J}$. Next we define the action of the $\mathbf{Z}_{3}$ twist $\Theta$ on these momenta as $\Theta E_{I}=\Theta_{I}^{J} E_{J}$ and $\Theta \widetilde{E}^{I}=\Theta^{I}{ }_{J} \widetilde{E}^{J}$, where

$$
\begin{gathered}
\Theta_{I}{ }^{J}=\left(\begin{array}{cccc}
0 & 1 & 0 & 0 \\
-1 & -1 & 0 & 0 \\
0 & 0 & 0 & 1 \\
0 & 0 & -1 & -1
\end{array}\right), \\
\Theta_{J}^{I}=\left(\begin{array}{cccc}
-1 & 1 & 0 & 0 \\
-1 & 0 & 0 & 0 \\
0 & 0 & -1 & 1 \\
0 & 0 & -1 & 0
\end{array}\right) .
\end{gathered}
$$

Note that they are block diagonal and $\Theta^{I}{ }_{J}=\left(\Theta^{-1}\right)_{J}{ }_{J}$.

An orbifold twist must leave the conformal dimensions invariant; that is, under the $\Theta$ twist

$$
P_{L, R} \rightarrow P_{L, R}^{\prime},
$$

we must have $P_{L}^{2} / 2=\left(P_{L}^{\prime}\right)^{2} / 2$ and $P_{R}^{2} / 2=\left(P_{R}^{\prime}\right)^{2} / 2$. This requirement imposes strong constraints on $B_{I J}$ and $G_{I J}$. It is straightforward to solve these constraints, and we have

$$
\begin{gathered}
2 B_{I J}=\left(\begin{array}{cccc}
0 & d & a & c \\
-d & 0 & -a-c & a \\
-a & a+c & 0 & b \\
-c & -a & -b & 0
\end{array}\right), \\
G_{I J}=\left(\begin{array}{cccc}
2 u & -u & f & h \\
-u & 2 u & -f-h & f \\
f & -f-h & 2 v & -v \\
h & f & -v & 2 v
\end{array}\right),
\end{gathered}
$$

where $2 B_{I J}$ are defined modulo 1 . Thus, the moduli space of the $\Gamma^{4,4}$ lattice with the $\mathbf{Z}_{3}$ (and the $\mathbf{Z}_{6}$ ) symmetry is eight dimensional (four parameters from $B_{I J}$ and the other four from $\left.G_{I J}\right)$. In the $N 0$ model, the gauge symmetry is $\mathrm{SU}(3)$ $\otimes R \otimes \mathrm{SO}(32)$, where the gauge symmetry group $R$ coming from $\Gamma^{4,4}$ depends on the values of the moduli. A generic point in the above moduli space has $R=\mathrm{U}(1)^{4}$. There are also special points in this moduli space with enhanced gauge symmetry. These are of particular interest since the $R=\mathrm{U}(1)^{4}$ leads to an empty messenger group $M$ in the corresponding $N=1$ model, which is phenomenologically unappealing. For this reason, we will confine our attention to a one-dimensional subspace of the above moduli space that includes the points of enhanced gauge symmetry. To describe this subspace it suffices to put $G_{11}=G_{33}=2$, $G_{13}=0 ; 2 B_{I J}=-\frac{1}{2} G_{I J}$ if $I>J$, and $2 B_{I J}=\frac{1}{2} G_{I J}$ if $I<J$. So there is only one free parameter left, $h \equiv G_{14}$. Let us con- sider the region $0 \leqslant h \leqslant 1$. We may express the vectors $E_{I}$ (and $\widetilde{E}^{I}$ ) in terms of the SU(3) root and weight vectors $e_{i}$ and $\tilde{e}^{i}$ :

$$
\begin{gathered}
E_{1}=\left(e_{1}, 0\right), \quad E_{2}=\left(e_{2}, 0\right), \quad E_{3}=\left(-h \widetilde{e}^{2}, g e_{1}\right) \\
E_{4}=\left(h \widetilde{e}^{1}, g e_{2}\right), \\
\widetilde{E}^{1}=\left(\widetilde{e},-h e_{2} / 3 g\right), \quad \widetilde{E}^{2}=\left(\widetilde{e}^{2}, h e_{1} / 3 g\right) \\
\widetilde{E^{3}}=(0, \widetilde{e} / g), \quad \widetilde{E}^{4}=\left(0, \widetilde{e}^{2} / g\right)
\end{gathered}
$$

Here $g \equiv \sqrt{1-h^{2} / 3}$. Note that the states with $P_{R}=0$ and $P_{L}^{2}=2$ are massless. For $h \neq 0,1$ the only solutions to these conditions are those with $P_{L}=E_{I} N^{I}, N^{3}, N^{4}=0$, which give six gauge bosons of SU(3). The other two gauge bosons are the Cartan generators, coming from the oscillator excitations. There are two more gauge bosons coming from the oscillator excitations of the other two world-sheet bosons. So for $0<h<1$, we have $R=\mathrm{SU}(3) \otimes \mathrm{U}(1)^{2}$. For $h=0$ the gauge symmetry is enhanced to $\mathrm{SU}(3)^{2}$, as $N^{3}$ and $N^{4}$ in Eq. (6.1) need no longer be zero for the states to be massless. Another special point of enhanced gauge symmetry is $h=1$ where $R=\mathrm{SO}(8)$. This can be readily verified by writing the irreps of $\mathrm{SO}(8)$ in the $\mathrm{SU}(3) \otimes \mathrm{U}(1)^{2}$ basis (4.11).

So the $N 0$ model has gauge symmetry $\mathrm{SU}(3)$ $\otimes R \otimes \mathrm{SO}(32)$, where $R=\mathrm{SU}(3)^{2}, \mathrm{SU}(3) \otimes \mathrm{U}(1)^{2}$, and $\mathrm{SO}(8)$ for $h=0,0<h<1$, and $h=1$, respectively. Away from this one-dimensional moduli space, $R=U(1)^{4}$ generically. Next we introduce the Wilson lines that break the gauge symmetry to $\mathrm{SU}(3) \otimes R \otimes \mathrm{SO}(10)_{3} \otimes \mathrm{U}(1)$, where $R$ is untouched:

$$
\begin{aligned}
& V_{1}=\left[0\left(0 e_{1} / 2\right)\left(0 a_{1}\right)\left(0 b_{1}\right)|| 0 c_{1} d_{1}|\mathbf{s 0 0}| C\right], \\
& V_{2}=\left[0\left(0 e_{2} / 2\right)\left(0 a_{2}\right)\left(0 b_{2}\right)|| 0 c_{2} d_{2}|\mathbf{0 s 0}| C\right] .
\end{aligned}
$$

Here $\left(a_{1}, b_{1}\right)=\left(-h \widetilde{E}^{4}+E_{1}+E_{3}\right) / 2,\left(c_{1}, d_{1}\right)=-h \widetilde{E}^{4} / 2$ for $0 \leqslant h \leqslant 1 / 2$ and $\left(a_{1}, b_{1}\right)=\left((h-1) \widetilde{E}^{4}-E_{1}+E_{3}\right) / 2,\left(c_{1}, d_{1}\right)$ $=(h-1) \widetilde{E}^{4} / 2$ for $1 / 2<h \leqslant 1$. Similarly, $\left(a_{2}, b_{2}\right)=\left(h \widetilde{E}^{3}\right.$ $\left.+E_{2}+E_{4}\right) / 2, \quad\left(c_{2}, d_{2}\right)=h \widetilde{E}^{3} / 2$ for $0 \leqslant h \leqslant 1 / 2 \quad\left(a_{2}, b_{2}\right)$ $=\left[-(h-1) \widetilde{E}^{3}-E_{2}+E_{4}\right] / 2, \quad\left(c_{2}, d_{2}\right)=-(h-1) \widetilde{E}^{3} / 2$ for $1 / 2<h \leqslant 1$. Mathematically, this definition of the Wilson lines $V_{1}$ and $V_{2}$ is discontinuous. Physically, however, there is no discontinuity as the latter belongs to $\Gamma^{6,22}$. (Recall that a lattice shift is defined up to a lattice spacing as the latter does not affect the corresponding orbifold model.) Using exactly the same $\mathbf{Z}_{3} \otimes \mathbf{Z}_{2}$ orbifold set $\left\{V_{i}, k_{i j}\right\}$ given in Sec. IV, we obtain the corresponding three-family $\mathrm{SO}(10)$ grand unified model with gauge symmetry $\mathrm{SU}(2)_{1} \otimes \mathrm{U}(1)$ $\otimes M \otimes \mathrm{SO}(10)_{3} \otimes \mathrm{U}(1)$. Here $M=\mathrm{U}(1)^{2}, \mathrm{U}(1)$, and $\mathrm{SU}(2)$ 3 $\otimes \mathrm{U}(1)$ for $R=\mathrm{SU}(3)^{2}, \mathrm{SU}(3) \otimes \mathrm{U}(1)^{2}$, and $\mathrm{SO}(8)$, respectively. For $R=U(1)^{4}, M$ is empty. The $h=1$ point with $\mathrm{SO}(8)$ has been analyzed in great detail already. The massless spectra of the $M=U(1)^{2}$ model, the $M=U(1)$ model, and the empty $M$ model are given in Table II.

Note that we can also obtain the $M=U(1)$ model by giving a nonzero vacuum expectation value to the appropriate Higgs field in either the $M=\mathrm{SU}(2)_{3} \otimes \mathrm{U}(1)$ model [the 
$(\mathbf{1 , 2}, \mathbf{1})(0,-3,0)]$ or the $M=\mathrm{U}(1)^{2}$ model $[(\mathbf{1}, \mathbf{1})(0,0$, -6,0)]. Similarly, the empty $M$ model can be obtained from the $M=\mathrm{U}(1)$ model via spontaneous symmetry breaking in the corresponding effective field theory. We may also obtain $\mathrm{SU}(5)$ grand unified models by giving the $\mathrm{SO}(10)$ adjoint (and possibly other) Higgs fields nonzero vacuum expectation values. A typical grand unification scale is a few orders of magnitude below the Planck scale. Presumably, the effective field theory approach is adequate if the vacuum expectation values are at the grand unification scale or below. When the vacuum expectation values approach the Planck or string scale, the string construction is clearly preferable. A more complete exploration of the full moduli space is clearly desirable.

The hidden sector SU(2) ${ }_{1}$ of the above set of models is asymptotically free while $M$ is not. At the string scale, the hidden $\mathrm{SU}(2)_{1}$ coupling $\alpha_{2}$ is 3 times that of the $\mathrm{SO}(10)_{3}$. So for typical values of the $\mathrm{SO}(10)$ grand unification coupling, $\alpha_{2}$ becomes large at a rather low scale, within a few orders of magnitude above the electroweak scale. If $M$ is empty, the hidden sector physics will have negligible impact on the physics in the visible sector. So this is phenomenologically undesirable. So, hopefully, $M$ is nontrivial at the string scale, and the spontaneous symmetry breakings of the $M$ sector occur at relatively low scales.

\section{ANOTHER SO(10) MODEL}

Next we turn to a variation of the above $\mathrm{SU}(2)_{1} \otimes \mathrm{SU}(2)$ ${ }_{3} \otimes \mathrm{SO}(10)_{3} \otimes \mathrm{U}(1)^{3}$ model. Consider the model generated by the following vectors acting on the $N 1$ model, in the same basis as in the last section:

$$
\begin{gathered}
V_{0}=\left[-\frac{1}{2}\left(-\frac{1}{2} 0\right)^{3}|| 0^{2} 0_{r} 0_{r} \mid 0^{5} 0_{r}^{5} 0_{r}\right], \\
V_{1}=\left[0\left(-\frac{1}{3} \frac{1}{3}\right)^{3}|| \frac{e_{1}}{3}\left(\frac{2}{3}\right) 0_{r} 0_{r} \mid\left(\frac{1}{3}\right)^{5} 0_{r}^{5}\left(\frac{2}{3}\right)_{r}\right], \\
V_{2}=\left[0(00)\left(-\frac{1}{2} \frac{1}{2}\right)^{2}|| \frac{e_{1}}{2} 00_{r}\left(\frac{\sqrt{2}}{2}\right) \mid 0_{r}^{5} 0_{r}^{5} 0_{r}\right], \\
W_{1}=\left[0\left(0 \frac{1}{2}\right)^{3}|| 0 \frac{1}{2} 0_{r} 0_{r} \mid\left(\frac{1}{2}\right)^{5} 0_{r}^{5} 0_{r}\right], \\
W_{2}=\left[0(00)\left(0 \frac{1}{2}\right)^{2}|| 0^{2} 0_{r} 0_{r} \mid 0^{5} 0_{r}^{5} 0_{r}\right] .
\end{gathered}
$$

This model has the same gauge symmetry $\mathrm{SU}(2)_{1}$ $\otimes \mathrm{SU}(2)_{3} \otimes \mathrm{SO}(10)_{3} \otimes \mathrm{U}(1)^{3}$. It differs from the above model by shifting the momentum lattice of the $\mathrm{SU}(3)$ boson instead of the last of the $\mathrm{SO}(8)$ bosons. As a consequence, this model has a spectrum very similar to that of the one we discussed earlier, but the assignments of the $M$ and $\mathrm{U}(1)$ quantum numbers are different. Without going into details, we will simply make a few comments on this model. Note that the $V_{2}$ vector is the same as that in Eq. (5.2) and, therefore, its effect on the $N 1$ model is the same as before. The $V_{1}$ vector is different from that in Eq. (5.2), however. It breaks the original gauge group $\mathrm{SU}(3) \otimes \mathrm{SO}(8)$ $\otimes \mathrm{SO}(10)^{3} \otimes \mathrm{SO}(2)$ to $\mathrm{U}(1)^{2} \otimes G_{2} \otimes \mathrm{SO}(10)_{3} \otimes \mathrm{U}(1)$. The $G_{2}$ gauge bosons can be seen as follows. The $\mathrm{SU}(2)^{4}$ gauge group is reduced to $\mathrm{SU}(2)_{3} \otimes \mathrm{SU}(2)_{1}$. But there are addi- tional states coming from the original $(\mathbf{2 , 2 , 2 , 2 )}$ of the $\mathrm{SO}(8)$ gauge bosons [see Eq. (5.1)]. The only states that survive the $\mathbf{Z}_{3}$ projection are those with the $(\mathbf{4 , 2})$ quantum number [in $\left.\mathrm{SU}(2)_{3} \otimes \mathrm{SU}(2)_{1}\right]$. The above gauge bosons form the adjoint of $G_{2}\left[\supset \mathrm{SU}(2)_{3} \otimes \mathrm{SU}(2)_{1}\right]: \mathbf{1 4}=(\mathbf{3}, \mathbf{1})+(\mathbf{1}, \mathbf{3})+(\mathbf{4 , 2})$. The further $\mathbf{Z}_{2}$ projection removes the $(\mathbf{4 , 2})$ states, and we are left with the $\mathrm{SU}(2)_{3} \otimes \mathrm{SU}(2)_{1}$ subgroup of $G_{2}$. The number of chiral generations in the $T 3$ and $T 6$ sectors is still $5_{L}$ and $2_{R}$. The number of the $\mathrm{SU}(2)_{1}$ doublets is still 6 , so that this subgroup (which is still the hidden sector) is asymptotically free. The complete massless spectrum of this model is given in the second column in Table I.

Note that both the hidden $\mathrm{SU}(2)_{1}$ sector as well as the $\mathrm{SU}(2)_{3}$ in the $M$ sector in this model comes from the $\mathrm{SO}(8)$ lattice in the original $N 1$ model. In the moduli space discussed in the previous section, we see that $\mathrm{SO}(8)$ is a special point. When we move away from that point in the moduli space, the gauge symmetry $\mathrm{SU}(2)_{1} \otimes \mathrm{SU}(2)_{3}$ in the final model will be reduced. In that case, the biggest non-Abelian gauge group one can get is $\mathrm{SU}(2)_{4}$, not very interesting phenomenologically since it turns out not to be asymptotically free. One may gain further insight into the relation between this and the first $\mathrm{SO}(10)$ models by considering a more general moduli space.

Let us make another comment concerning the construction of this model. The above construction, i.e., the set $\left\{V_{i}, k_{i j}\right\}$, satisfies the simplified rules. Then the condition (3.24) follows. This same model may be obtained by a different set of $\left\{V_{i}, k_{i j}\right\}$. This alternative set turns out not to satisfy the simplified rules. So in that case we must use the full set of rules. This issue is discussed in Appendix B.

\section{THIRD SO(10) MODEL}

Again we start with with a Narain model, called the $N 2$ model, with $\Gamma^{6,22}=\Gamma^{6,6} \otimes \Gamma^{16}$, where $\Gamma^{16}$ is the $\operatorname{spin}(32) / \mathbf{Z}_{2}$ lattice and $\Gamma^{6,6}$ is the $\mathrm{E}_{6}$ lattice, i.e., $p_{R}, p_{L}$ $\in \widetilde{\Gamma}^{6}\left(\mathrm{E}_{6}\right.$ weight lattice), and $p_{L}-p_{R} \in \Gamma^{6}\left(\mathrm{E}_{6}\right.$ root lattice). We choose this particular lattice for later convenience. Recall that, under $\mathrm{E}_{6} \supset \mathrm{SU}(3)^{3}$,

$$
\begin{gathered}
\mathbf{2 7}=(\mathbf{3}, \mathbf{3}, \mathbf{1})+(\overline{\mathbf{3}}, \mathbf{1}, \mathbf{3})+(\mathbf{1}, \overline{\mathbf{3}}, \overline{\mathbf{3}}), \\
\mathbf{7 8}=(\mathbf{8}, \mathbf{1}, \mathbf{1})+(\mathbf{1}, \mathbf{8}, \mathbf{1})+(\mathbf{1}, \mathbf{1}, \mathbf{8})+(\mathbf{3}, \overline{\mathbf{3}}, \mathbf{3})+(\overline{\mathbf{3}}, \mathbf{3}, \overline{\mathbf{3}}),
\end{gathered}
$$

and so we can write $\mathbf{p} \in \Gamma^{6}$ as

$$
\mathbf{p}=\left(\mathbf{q}_{1}+a \mathbf{w}_{1}, \mathbf{q}_{2}+a \overline{\mathbf{w}}_{2}, \mathbf{q}_{3}+a \mathbf{w}_{3}\right),
$$

where $\mathbf{q}_{i} \in \Gamma^{2}\left[\mathrm{SU}(3)\right.$ root lattice], $\mathbf{w}_{i}\left(\overline{\mathbf{w}}_{i}\right)$ is in the $\mathbf{3}(\overline{\mathbf{3}})$ weight of $\mathrm{SU}(3)$, and $a=0,1,2$. To convert this model to a $N=4$ supersymmetric model with gauge symmetry $\mathrm{SU}(3)^{2} \otimes \mathrm{U}(1)^{2} \otimes \mathrm{SO}(10)^{3} \otimes \mathrm{SO}(2)$, we introduce, in addition to $V_{0}$, two Wilson lines,

$$
\begin{aligned}
& V_{1}=\left[0\left(0 \frac{e_{1}}{2}\right)^{2}(00)|| 0^{2} \frac{e_{1}}{2}|\mathbf{s 0 0}| C\right], \\
& V_{2}=\left[0\left(0 \frac{e_{2}}{2}\right)^{2}(00)|| 0^{2} \frac{e_{2}}{2}|\mathbf{0 s 0}| C\right],
\end{aligned}
$$


where $e_{i}$ are $\mathrm{SU}(3)$ roots, $\mathbf{s}$ is a component in the spinor weight of $\mathrm{SO}(10)$, and $C=-1 / 2$ in $\mathrm{SO}(2)$. Under the action of these Wilson lines, we see that states with $a=1,2$ in Eq. (8.3) are removed, and the $\mathrm{E}_{6}$ gauge symmetry is broken down to $\mathrm{SU}(3)^{2} \otimes \mathrm{U}(1)^{2}$. Since no new gauge bosons are introduced by the other sectors, we end up with the gauge symmetry $\mathrm{SU}(3)^{2} \otimes \mathrm{U}(1)^{2} \otimes \mathrm{SO}(10)^{3} \otimes \mathrm{SO}(2)$. The model remains $N=4$ supersymmetric, since the Wilson lines do not break supersymmetry. Let us call this the $N 3$ model.

To obtain the three-family $\mathrm{SO}(10)$ model, we introduce the following set of vectors to act on the $N 3$ model:

$$
\begin{gathered}
V_{0}=\left[-\frac{1}{2}\left(-\frac{1}{2} 0\right)^{3}|| 0^{3} \mid 0^{5} 0_{r}^{5} 0_{r}\right], \\
V_{1}=\left[0\left(-\frac{1}{3} \frac{1}{3}\right)^{3}|| 0 \frac{e_{1}}{3} \frac{2}{3} \mid\left(\frac{1}{3}\right)^{5} 0_{r}^{5}\left(\frac{2}{3}\right)_{r}\right], \\
V_{2}=\left[0(00)\left(-\frac{1}{2} \frac{1}{2}\right)^{2}||\left(\frac{e_{1}}{2}\right)^{2} 0 \mid 0^{5} 0_{r}^{5} 0_{r}\right], \\
W_{1}=\left[0\left(0 \frac{1}{2}\right)^{3}|| 00 \frac{1}{2} \mid\left(\frac{1}{2}\right)^{5} 0_{r}^{5} 0_{r}\right], \\
W_{2}=\left[0(00)\left(0 \frac{1}{2}\right)^{2}|| 0^{3} \mid 0^{5} 0_{r}^{5} 0_{r}\right] .
\end{gathered}
$$

Here, it is important to specify the basis the above vectors are acting on. As before, the $\mathrm{SO}(10)$ bosons are in the basis (4.13). The new feature is the basis of the right-moving bosons in $\Gamma^{6,6}$, namely, $X_{1}, X_{2}$, and $X_{3}$. The above vectors act on the basis $X_{+}, X_{-}$, and $X_{3}$, where

$$
X_{ \pm}=\frac{1}{\sqrt{2}}\left(X_{1} \pm X_{2}\right) \text {. }
$$

This means that $V_{2}$ acts as a $\mathbf{Z}_{2}$ outer automorphism on the first two right-moving complex bosons. The final gauge symmetry of this model is $\mathrm{SU}(2)_{1} \otimes \mathrm{SO}(10)_{3} \otimes \mathrm{U}(1)^{4}$. It is now straightforward to work out its massless spectrum of this, which is given in the third column in Table I.

\section{IX. $\mathrm{E}_{6}, \mathrm{SU}(\mathbf{5})$, AND OTHERS}

$\mathrm{E}_{6}$ grand unified string models were recently constructed by Erler [10]. His models involve $\left(\mathrm{E}_{6}\right)_{2}$ models with an even number of families. To obtain an odd number of families, it is natural to go to level 3. Below is a brief description of a three-family $\left(\mathrm{E}_{6}\right)_{3}$ model, which is closely related to the third $\mathrm{SO}(10)$ model given in Sec. VIII. We shall first construct a $N=4$ supersymmetric model with gauge symmetry $\mathrm{SU}(3)^{2} \otimes \mathrm{E}_{6}^{3}$, and then orbifold it. We shall also show how three-family $\mathrm{SU}(5)$ models can be obtained from the above $\mathrm{SO}(10)$ models. On the way, we shall also present a threefamily SU(6) model.

Let us start with the above $N 2$ model, with $\Gamma^{6,22}=\Gamma^{6,6} \otimes \Gamma^{16}$, where $\Gamma^{16}$ is the $\operatorname{spin}(32) / \mathbf{Z}_{2}$ lattice, and $\Gamma^{6,6}$ is the $\mathrm{E}_{6}$ lattice. To convert this model to a model with gauge symmetry $\mathrm{SU}(3)^{2} \otimes \mathrm{E}_{6}^{3}$, we introduce, besides $V_{0}$, two Wilson lines,

$$
\begin{aligned}
& V_{1}=\left(0(00)^{3}|| 0^{2} \frac{e_{1}}{2}|\mathbf{s} \mathbf{0 0}| C\right), \\
& V_{2}=\left(0(00)^{3}|| 0^{2} \frac{e_{2}}{2}|\mathbf{0 s 0}| C\right) .
\end{aligned}
$$

The left-moving parts of these two Wilson lines are identical to the Wilson lines (8.4) introduced above. Again, the gauge bosons corresponding to the states with $a=1,2$ in Eq. (8.3) are projected out. So the gauge bosons in the $\alpha V=\mathbf{0}$ sector form the gauge symmetry $\mathrm{SU}(3)^{2} \otimes U(1)^{2} \otimes \mathrm{SO}(10)^{3}$ $\otimes \mathrm{SO}(2)$, just like the $N 3$ model above. The difference comes from the introduction of additional gauge bosons by the new sectors. Recall that under $\mathrm{E}_{6} \supset \mathrm{SO}(10) \otimes \mathrm{U}(1)$,

$$
\mathbf{7 8}=\mathbf{1}(0)+\mathbf{4 5}(0)+\mathbf{1 6}(3)+\overline{\mathbf{1 6}}(-3) .
$$

It is easy to see that the $V_{1}, V_{2}$, and $V_{1}+V_{2}$ sectors provide the necessary $\mathbf{1 6}(3)$ and $\overline{\mathbf{1 6}}(-3)$ gauge bosons to the three $\mathrm{SO}(10)$ 's, respectively. [A little care is needed to disentangle the three U(1)'s]. So the resulting model, to be referred to as the $N 4$ model, has a gauge symmetry $\mathrm{SU}(3)^{2} \otimes \mathrm{E}_{6}^{3}$.

Next, we perform a $\mathbf{Z}_{6}$ orbifold on this $\mathrm{E}_{6}^{3}$ model; i.e., we let the set of vectors (8.6) act on this $N 4$ model. Now $V_{1}$, i.e., the $\mathbf{Z}_{3}$ twist, on $\left(\mathrm{E}_{6}\right)^{3}$ converts it to $\left(\mathrm{E}_{6}\right)_{3}$, yielding nine chiral families, i.e., $\mathbf{2 7}_{L}$ of $\left(\mathrm{E}_{6}\right)_{3}$. Again, $V_{2}$ cuts the nine left-handed families to five, while introducing two righthanded families. The massless spectrum of this $\mathrm{E}_{6}$ model is shown in the first column in Table III. The analysis is very similar to earlier discussions, and so we shall not repeat them. Instead, we shall make a number of related remarks.

We note that the third $\mathrm{SO}(10)_{3}$ model constructed above can also be obtained from this $\mathrm{E}_{6}$ model by spontaneous symmetry breaking. Classically, a typical vacuum expectation value of the adjoint Higgs representation of the $\mathrm{E}_{6}$ model lies in a flat direction. Choosing an appropriate vacuum expectation value breaks the $\mathrm{E}_{6}$ gauge symmetry to $\mathrm{SO}(10) \otimes \mathrm{U}(1)$, yielding the third $\mathrm{SO}(10)_{3}$ model given in the third column in Table I.

Note that we may reach the $N 4$ model via other paths. For example, we may start with $\Gamma^{6,22}=\left(\Gamma^{2,2}\right)^{3} \otimes\left(\Gamma^{8}\right)^{2}$, where $\Gamma^{2,2}$ is the same SU(3) lattice in the $N 0$ model and $\Gamma^{8}$ is the $\mathrm{E}_{8}$ lattice, and then introduce, besides $V_{0}$, the vector

$$
\begin{aligned}
V_{1}= & {\left[0(00)^{3}|| 0^{2} \widetilde{e}^{-1}\left|\left(\frac{1}{3}\right)_{r}\left(\frac{1}{3}\right)_{r}\left(\frac{2}{3}\right)_{r} 0_{r}^{5}\right|\left(-\frac{1}{3}\right)_{r}\left(-\frac{1}{3}\right)_{r}\right.} \\
& \left.\times\left(-\frac{2}{3}\right)_{r} 0_{r}^{5}\right] .
\end{aligned}
$$

This $V_{1}$ may be rewritten in a more suggestive way in the $\mathrm{E}_{8} \supset \mathrm{E}_{6} \otimes \mathrm{SU}(3)$ basis:

$$
V_{1}=\left[0(00)^{3}|| 0^{2} \widetilde{e}^{-1}\left|\left(-\tilde{e}^{-1}\right) \mathbf{0}\right| \tilde{e}^{-1} \mathbf{0}\right],
$$

where the $\mathbf{0}$ indicates the identity weight of $\mathrm{E}_{6}$. The $\mathbf{0}$ sector gives rise to the gauge bosons of $\mathrm{SU}(3)^{2} \otimes \mathrm{SU}(3)^{3}$ $\otimes \mathrm{E}_{6} \otimes \mathrm{E}_{6}$. The $V_{1}$ and $2 V_{1}$ sectors supply the remaining gauge bosons $(\mathbf{3}, \overline{\mathbf{3}}, \mathbf{3})$ and $(\overline{\mathbf{3}}, \mathbf{3}, \overline{\mathbf{3}})$ in $\mathrm{SU}(3)^{3}$, and so the final gauge symmetry is $\mathrm{SU}(3)^{2} \otimes \mathrm{E}_{6}^{3}$.

In each of the above $\mathrm{SO}(10)$ models, we may also introduce a Wilson line to break it to SU(5). This is the string equivalence of spontaneous symmetry breaking. To be specific, we can add to any of the above sets of Wilson lines the vector

$$
V_{3}=\left[0(00)^{3}|| \widetilde{e}^{2} 0^{2}\left|\left(\frac{1}{3} \frac{1}{3} \frac{1}{3} \frac{1}{3} \frac{2}{3}\right)_{r}^{3}\right| 0_{r}\right] .
$$

Acting on any of the $N=4$ supersymmetric models that contain the gauge symmetry $\mathrm{SO}(10)^{3}$, i.e., the $N 1$ or the $N 3$ model, this Wilson line breaks $\mathrm{SO}(10)$ to $\mathrm{SU}(5) \otimes \mathrm{U}(1)$. To 
illustrate this construction, let us consider the case where this Wilson line is added to the set (8.4) acting on the $\mathrm{E}_{6} \otimes \mathrm{SO}(32)$ model. This results in the $N 5$ model, i.e., an $N=4$ supersymmetric model with gauge symmetry $\mathrm{SU}(3)^{2}$ $\otimes \mathrm{SU}(5)^{3} \otimes \mathrm{U}(1)^{6}$. Now, we let the set (8.6) of twists act on this $N 5$ model. This results in a three-family SU(5) model, with gauge symmetry $\mathrm{SU}(2)_{1} \otimes \mathrm{SU}(5)_{3} \otimes U(1)^{5}$. Its massless spectrum is given in the third column in Table III. It is just as easy to add the Wilson line $V_{3}$ to the other cases to break $\mathrm{SO}(10)_{3}$ to $\mathrm{SU}(5)_{3} \otimes \mathrm{U}(1)$.

If we add the $V_{3}$ Wilson line (9.6) to the set of Wilson lines (9.1) and (9.2) that yields the $\mathrm{E}_{6}$ model, we get an $\mathrm{SU}(6)$ model instead, since this Wilson line happens to break $\mathrm{E}_{6}$ to $\mathrm{SU}(6) \otimes \mathrm{U}(1)$. So after the $\mathbf{Z}_{6}$ twist (8.6) acting on this model, the final orbifold model is a three-family $\mathrm{SU}(6)$ model, with gauge symmetry $\mathrm{SU}(2)_{1} \otimes \mathrm{SU}(2)_{3} \otimes \mathrm{SU}(6)_{3}$ $\otimes \mathrm{U}(1)^{3}$. The massless spectrum of this model is given in the second column in Table III. Note that the (semi)hidden sector is enhanced in this model compared with the $\mathrm{E}_{6}$ model that we started with. Also note that the spectrum of this $\mathrm{SU}(6)$ model is similar to that of the $\mathrm{SO}(10)$ model given in the second column of Table I. In particular, the massless spectrum of the SU(6) model can be obtained from that of the $\mathrm{SO}(10)$ model via replacing $\mathrm{SO}(10)_{3} \otimes \mathrm{U}(1)$ [the last $\mathrm{U}(1)$ ] by $\mathrm{SU}(6) \otimes \mathrm{U}(1)$ [the last $\mathrm{U}(1)$ ]. Similarly, if in the Wilson line (9.6) we shifted (by $\vec{e}^{2}$ ) not the first entry but the second one, we would get an SU(6) model whose spectrum can be obtained from that of the $\mathrm{SO}(10)$ model given in the first column of Table I via replacing $\mathrm{SO}(10)_{3} \otimes \mathrm{U}(1)$ by $\mathrm{SU}(6) \otimes \mathrm{U}(1)$.

Adding certain Wilson lines can sometimes enhance the hidden or messenger sector gauge group (while changing the observable sector gauge group). For example, if instead of the above $V_{3}$ Wilson line we add

$$
V_{3}=\left[0(00)^{3}|| \widetilde{e}^{2}\left(-\widetilde{e}^{2}\right) 0\left|\left(\frac{1}{3} \frac{1}{3} \frac{1}{3} \frac{1}{3} \frac{2}{3}\right)_{r}^{3}\right| 0_{r}\right]
$$

to the $N 1$ model, the resulting Narain model will have the gauge group $\mathrm{SU}(6) \otimes \mathrm{SU}(5)^{3} \otimes \mathrm{U}(1)^{5}$, and the corresponding $\mathbf{Z}_{6}$ orbifold model [whose observable sector would contain the gauge group $\left.\mathrm{SU}(5)_{3}\right]$ will have an enhanced hidden or messenger sector. This may be important for phenomenology, in particular, supersymmetry breaking in the hidden sector. The analysis of such models is underway and will be reported elsewhere.

If one prefers, one may modify the above Wilson lines (or add new Wilson lines) to break the grand unified gauge group further to $\mathrm{SU}(3) \otimes \mathrm{SU}(2) \otimes \mathrm{U}(1)$. One may also choose to break $\mathrm{SO}(10)$ to $\mathrm{SU}(2)^{2} \otimes \mathrm{SU}(4)$. This can also be done by giving the adjoint Higgs appropriate expectation values in the effective field theory. By now, the procedure should be clear.

\section{SUMMARY AND REMARKS}

We have seen that the rules for asymmetric orbifold model building can be considered as a generalization of those for the free fermionic string models. These rules are summarized at the end of Sec. III. They are useful to keep track of the various phases in the partition function, and become very handy for working out the spectrum when the orbifolds get complicated. Using these rules, we construct the three-family grand unified string models. We consider three types of $\mathbf{Z}_{6}$ orbifolds, namely, the three sets of vectors (4.12), (7.1), and (8.6), each acting on an appropriate $N=4$ supersymmetric model. If the $N=4$ model has gauge group $\mathrm{SO}(10)^{3}\left[\mathrm{SU}(5)^{3}\right]$, the resulting model had a gauge group $\mathrm{SO}(10)_{3}\left[\mathrm{SU}(5)_{3}\right]$. The $\mathrm{SO}(10)_{3}$ models with an asymptotically free hidden sector [namely, $\left.\mathrm{SU}(2)_{1}\right]$ are presented in Tables I and II. Using the third set of vectors (8.6), we also obtain a three-family $\left(\mathrm{E}_{6}\right)_{3}$ model and a three-family $\mathrm{SU}(6)_{3}$ model. They are presented in Table III. Other models with smaller gauge groups can be obtained from the above models via either the string approach (i.e., changing moduli or Wilson lines) or the field theory approach (i.e., spontaneous symmetry breaking). We give such an $\mathrm{SU}(5)$ example in Table III.

Although the only way to obtain level-3 SO(10) current algebra is via an outer automorphism used in our construction, it has been pointed out that $\mathrm{SO}(10)$ at higher levels (say, $K=4$ ) can be constructed via different (more "economical") embeddings [15]. However, it seems unlikely that we can get a three-family $\mathrm{SO}(10)_{4}$ model, especially if we impose any of the other rather weak phenomenological requirements. So a distinct feature of grand unification in string theory is the very limited number of possibilities. At the same time, the models are quite complicated. This complicated structure hopefully bodes well for phenomenology.

There are still a number of important questions concerning grand unification in string theory. In particular, the complete moduli space that has a grand unified gauge symmetry with three families, an asymptotically free hidden sector, and an intermediate or messenger sector should be classified. The three types of asymmetric orbifolds considered in this work may not be exhaustive. Also, the couplings in each case should be worked out, following the method already developed [16]. Both problems are relatively straightforward. A more challenging problem is the string dynamics. With recent advances in understanding nonperturbative dynamics, presumably this is also within reach.

\section{ACKNOWLEDGMENTS}

This work was supported in part by the National Science Foundation.

\section{APPENDIX A: WORLD-SHEET FERMIONS AND BOSONS}

\section{Free fermions}

Consider a single free left-moving complex fermion with the monodromy

$$
\psi_{v}\left(z e^{2 \pi i}\right)=e^{-2 \pi i v} \psi_{v}(z), \quad-\frac{1}{2} \leqslant v<\frac{1}{2} .
$$

The field $\psi_{v}(z)$ has the mode expansion

$$
\psi_{v}(z)=\sum_{n=1}^{\infty}\left\{b_{n+v-1 / 2} z^{-(n+v)}+d_{n-v-1 / 2}^{\dagger} z^{n-v-1}\right\} .
$$

Here $b_{r}^{\dagger}$ and $d_{s}^{\dagger}$ are creation operators, and $b_{r}$ and $d_{s}$ are annihilation operators. The quantization conditions read 
$\left\{b_{r}^{\dagger}, b_{r^{\prime}}\right\}=\delta_{r r^{\prime}}, \quad\left\{d_{s}^{\dagger}, d_{s^{\prime}}\right\}=\delta_{s s^{\prime}}$, others vanish.

The Hamiltonian $H_{v}$ and fermion number operator $N_{v}$ are given by

$$
\begin{aligned}
H_{v}= & \sum_{n=1}^{\infty}\left\{\left(n+v-\frac{1}{2}\right) b_{n+v-1 / 2}^{\dagger} b_{n+v-1 / 2}\right. \\
& \left.+\left(n-v-\frac{1}{2}\right) d_{n-v-1 / 2}^{\dagger} d_{n-v-1 / 2}\right\}+\frac{v^{2}}{2}-\frac{1}{24}, \\
N_{v}= & \sum_{n=1}^{\infty}\left\{b_{n+v-1 / 2}^{\dagger} b_{n+v-1 / 2}-d_{n-v-1 / 2}^{\dagger} d_{n-v-1 / 2}\right\} .
\end{aligned}
$$

Note that the vacuum energy is $v^{2} / 2-\frac{1}{24}$. Also note that for a Ramond fermion $(v=-1 / 2)$ the vacuum is degenerate: There are two ground states $|0\rangle$ and $b_{0}^{\dagger}|0\rangle$.

The operator $-N_{v}$ is the generator of $\mathrm{U}(1)$ rotations. The corresponding characters read

$$
\begin{aligned}
Z_{u}^{v} & =\operatorname{Tr}\left[q^{H_{v}} g^{-1}(u)\right]=\operatorname{Tr}\left[q^{H_{v}} \exp \left(-2 \pi i u N_{v}\right)\right] \\
& =q^{v^{2} / 2-1 / 24} \prod_{n=1}^{\infty}\left(1+q^{n+v-1 / 2} e^{-2 \pi i u}\right)\left(1+q^{n-v-1 / 2} e^{2 \pi i u}\right) .
\end{aligned}
$$

Under the generators of modular transformations $[q=\exp (2 \pi i \tau)]$,

$$
S: \tau \rightarrow-1 / \tau, \quad T: \tau \rightarrow \tau+1,
$$

the characters (A6) transform as

$$
\begin{gathered}
Z_{u}^{v} \stackrel{S}{\rightarrow} e^{2 \pi i v u} Z_{-v}^{u}, \\
Z_{u}^{v} \stackrel{T}{\rightarrow} e^{2 \pi i\left(v^{2} / 2-1 / 24\right)} Z_{u-v-1 / 2}^{v} .
\end{gathered}
$$

In the cases where $v=-1 / 2$ (Ramond fermion) or $v=0$ (Neveu-Schwarz fermion) in Eq. (A1) the complex fermion $\psi_{v}(z)$ can be represented in terms of a linear combination of two real fermions. The corresponding characters for real fermions then are square roots of the characters $Z_{u}^{v}$ for the complex fermions ( $v$ and $u$ being $-1 / 2$ or 0 ). A more detailed discussion of the real fermion characters is given in [7].

\section{Twisted bosons}

Consider a single free left-moving complex boson with the monodromy

$$
\partial \phi_{v}\left(z e^{2 \pi i}\right)=e^{-2 \pi i v} \partial \phi_{v}(z), \quad 0 \leqslant v<1 .
$$

The field $\partial \phi_{v}(z)$ has the mode expansion

$$
\begin{aligned}
i \partial \phi_{v}(z)= & \delta_{v, 0} p z^{-1}+\left(1-\delta_{v, 0}\right) \sqrt{v} b_{v} z^{-v-1} \\
& +\sum_{n=1}^{\infty}\left\{\sqrt{n+v} b_{n+v} z^{-n-v-1}\right. \\
& \left.+\sqrt{n-v} d_{n-v}^{\dagger} z^{n-v-1}\right\} .
\end{aligned}
$$

Here $b_{r}^{\dagger}$ and $d_{s}^{\dagger}$ are creation operators, and $b_{r}$ and $d_{s}$ are annihilation operators. The quantization conditions read

$$
\begin{aligned}
& {\left[b_{r}, b_{r^{\prime}}^{\dagger}\right]=\delta_{r r^{\prime}}, \quad\left[d_{s}, d_{s^{\prime}}^{\dagger}\right]=\delta_{s s^{\prime}},} \\
& {\left[x^{\dagger}, p\right]=\left[x, p^{\dagger}\right]=i, \text { others vanish. }}
\end{aligned}
$$

The Hamiltonian $H_{v}$ and angular momentum operator $J_{v}$ are given by

$$
\begin{aligned}
H_{v}= & \delta_{v, 0} p p^{\dagger}+\left(1-\delta_{v, 0}\right) v b_{v}^{\dagger} b_{v} \\
& +\sum_{n=1}^{\infty}\left\{(n+v) b_{n+v}^{\dagger} b_{n+v}+(n-v) d_{n-v}^{\dagger} d_{n-v}\right\} \\
+ & \frac{v(1-v)}{2}-\frac{1}{12}, \\
J_{v}= & \delta_{v, 0} i\left(x p^{\dagger}-x^{\dagger} p\right)-\left(1-\delta_{v, 0}\right) b_{v}^{\dagger} b_{v} \\
& -\sum_{n=1}^{\infty}\left\{b_{n+v}^{\dagger} b_{n+v}-d_{n-v}^{\dagger} d_{n-v}\right\} .
\end{aligned}
$$

Note that the vacuum energy is $v(1-v) / 2-\frac{1}{24}$.

The operator $J_{v}$ is the generator of $\mathrm{U}(1)$ rotations. The corresponding characters read $(v+u \neq 0)$

$$
\begin{aligned}
X_{u}^{v}= & \operatorname{Tr}\left[q^{H_{v}} g^{-1}(u)\right]=\operatorname{Tr}\left[q^{H_{v}} \exp \left(2 \pi i u J_{v}\right)\right] \\
= & q^{v(1-v) / 2-1 / 12}\left[1-\left(1-\delta_{v, 0}\right) q^{v} e^{-2 \pi i u}\right]^{-1} \\
& \times \prod_{n=1}^{\infty}\left(1-q^{n+v} e^{-2 \pi i u}\right)^{-1}\left(1-q^{n-v} e^{2 \pi i u}\right)^{-1} .
\end{aligned}
$$

Under the generators of modular transformations the characters (A15) transform as

$$
\begin{aligned}
X_{u}^{v} \stackrel{S}{\rightarrow} & \left(2 \sin (\pi u) \delta_{v, 0}+[2 \sin (\pi v)]^{-1} \delta_{u, 0}\right. \\
& \left.+\left(1-\delta_{v u, 0}\right) e^{-2 \pi i(v-1 / 2)(u-1 / 2)}\right) X_{-v}^{u} \\
X_{u}^{v} & \stackrel{T}{\rightarrow} \exp \left[2 \pi i\left(\frac{v(1-v)}{2}-\frac{1}{12}\right)\right] X_{u-v}^{v}
\end{aligned}
$$

In the cases where $v=-1 / 2$ or $v=0$ in Eq. (A10), the complex boson $\phi_{v}(z)$ can be represented in terms of a linear combination of two real bosons. The corresponding characters for real bosons then are square roots of the characters $X_{u}^{v}$ for the complex bosons ( $v$ and $u$ being $-1 / 2$ or 0 ). The twisted boson characters with a different overall normalization were discussed in Ref. [17].

\section{Chiral lattices}

Consider $d$ free left-moving real bosons with the monodromy

$$
\phi_{v}^{I}\left(z e^{2 \pi i}\right)=\phi_{v}^{I}(z)+v^{I}
$$

where $I=1,2, \ldots, d$, and $v^{I}$ is the $I$ th component of the shift vector $v$. The field $\phi_{v}^{I}(z)$ has the mode expansion

$$
i \phi_{v}^{I}(z)=i x^{I}+\left(p^{I}+v^{I}\right) \ln (z)-\sum_{n \neq 0} \frac{1}{\sqrt{n}} a_{n}^{I} z^{-n} .
$$

Here $a_{n}^{I}, n>0$, are annihilation operators, and $a_{n}^{I}, n<0$, are creation operators. In the following the eigenvalues of the momentum operator $p^{I}$ are taken to span an even lattice $\Gamma^{d}$ (with metric $g_{i j}$ ). The quantization conditions read 


$$
\left[a_{n}^{I}, a_{n^{\prime}}^{J}\right]=\delta^{I J} \delta_{n n^{\prime}}, \quad\left[x^{I}, p^{J}\right]=i \delta^{I J}, \text { others vanish. }
$$

The Hamiltonian operator is given by

$$
H_{v}=\frac{(p+v)^{2}}{2}+\sum_{n=1}^{\infty} n a_{-n}^{I} a_{n}^{I}-\frac{d}{24} .
$$

The momentum operator $p$ is the generator of translations. The corresponding characters read

$$
\begin{aligned}
Y_{u}^{v} & =\operatorname{Tr}\left[q^{H_{v}} g^{-1}(u)\right]=\operatorname{Tr}\left[q^{H_{v}} \exp (-2 \pi i p u)\right] \\
& =\frac{1}{\eta^{d}(q)} \sum_{p \in \Gamma^{d}} q^{(p+v)^{2} / 2} \exp (-2 \pi i p u) .
\end{aligned}
$$

Let $w_{a} \in \widetilde{\Gamma}^{d}, a=1, \ldots, M-1$, be a set of vectors such that $\Gamma_{0}^{d} \oplus \Gamma_{1}^{d} \oplus \cdots \oplus \Gamma_{M-1}^{d}=\widetilde{\Gamma}^{d}$, where $w_{0}$ is the null vector $\left(w_{0}^{I} \equiv 0\right), \quad$ and $\quad \Gamma_{a}^{d} \equiv\left\{w_{a}+p \mid p \in \Gamma^{d}\right\}, \quad a=0,1, \ldots, M-1$. [Thus, $w_{a} \notin \Gamma^{d}$ for $a \neq 0$; also note that $M=\operatorname{det}\left(g_{i j}\right)$.] Consider the set of characters $Y_{u}^{v+w_{a}}$ :

$$
\begin{gathered}
Y_{u}^{v+w_{a}} \stackrel{T}{\rightarrow} \exp \left[2 \pi i\left(\frac{1}{2}\left(w_{a}+v\right)^{2}-\frac{d}{24}\right)\right] Y_{u-v}^{v+w_{a}}, \\
Y_{u}^{v+w_{a}} \stackrel{S}{\rightarrow} \sum_{b=0}^{M-1} S_{a b}(v, u) Y_{-v}^{u+w_{b}},
\end{gathered}
$$

where

$$
S_{a b}(v, u)=M^{-1 / 2} \exp \left[2 \pi i\left(w_{a}+v\right)\left(w_{b}+u\right)\right] .
$$

Let $N$ the smallest positive integer such that $\forall a N w_{a}^{2}$ $\in 2 \mathbf{Z}$. If $N=1$ [in which case $\Gamma^{d}$ is an even self-dual lattice with $\left.M=\operatorname{det}\left(g_{i j}\right)=1\right]$, we will use the characters $Y_{u}^{v}$ defined in Eq. (A22) whose modular transformations are particularly simple for $N=1$ :

$$
\begin{gathered}
Y_{u}^{v} \stackrel{T}{\rightarrow} \exp \left[2 \pi i\left(\frac{1}{2} v^{2}-\frac{d}{24}\right)\right] Y_{u-v}^{v}, \\
Y_{u}^{v} \stackrel{S}{\rightarrow} \exp (2 \pi i v u) Y_{-v}^{u} .
\end{gathered}
$$

If $N>1$, the set of characters $Y_{u}^{v+w_{a}}$ is such that the $T$ transformation is diagonal (with respect to $a$ ), whereas the $S$ transformation is not. There exists a basis such that both $S$ and $T$ transformations act as permutations. In particular, consider the case where $N$ is a prime. In the discussion of asymmetric orbifolds we will use the set of characters

$$
\begin{gathered}
Y_{\sigma, u}^{0, v} \equiv Y_{u}^{v}, \\
Y_{\sigma, u}^{\lambda, v} \equiv \sum_{a=0}^{M-1} \exp \left[-2 \pi i \lambda\left(u w_{a}+\frac{1}{2} \sigma w_{a}^{2}\right)\right] Y_{u}^{v+\lambda w_{a}}, \quad \lambda \neq 0,
\end{gathered}
$$

where $\lambda$ and $\sigma$ are integers taking values between 0 and $N-1$, such that $\lambda+\sigma \neq 0$. The modular transformation properties of $Y_{\sigma, u}^{\lambda, v}$ read

$$
Y_{\sigma, u}^{\lambda, v} \stackrel{T}{\rightarrow} \exp \left[2 \pi i\left(\frac{1}{2} v^{2}-\frac{d}{24}\right)\right] Y_{\sigma-\lambda, u-v}^{\lambda, v},
$$

$$
\begin{aligned}
Y_{\sigma, u}^{\lambda, v} \stackrel{S}{\rightarrow} & \left\{M^{-1 / 2} \delta_{\lambda, 0}+M^{1 / 2} \delta_{\sigma, 0}+\left(1-\delta_{\lambda \sigma, 0}\right)\right. \\
& \times \exp [2 \pi i \chi(\lambda, \sigma)]\} \exp (2 \pi i v u) Y_{-\lambda,-v}^{\sigma, u},
\end{aligned}
$$

where $Y_{\sigma, u}^{\lambda, v} \equiv Y_{\sigma, u}^{\lambda+N, v} \equiv Y_{\sigma+N, u}^{\lambda, v}$, and

$$
\begin{gathered}
\exp [2 \pi i \chi(\lambda, \sigma)] \equiv M^{-1 / 2} \sum_{a=0}^{M-1} \exp \left(-2 \pi i \frac{1}{2} \lambda \sigma w_{a}^{2}\right), \\
\lambda \sigma \neq 0 .
\end{gathered}
$$

Note that $\chi(\lambda, \sigma)$ are real numbers, and $\chi(\lambda, \lambda) \equiv-d / 8$.

To illustrate the above discussion we note that the root lattices of simply laced Lie groups are even. The groups that have prime $N$ are the following: (i) $\mathrm{SU}(n), n$ is an odd prime, and $N=n$; (ii) $\mathrm{E}_{6}, N=3$; (iii) $\mathrm{SO}(8 n), N=2$; and (iv) $\mathrm{E}_{8}, N=1$.

Similar considerations apply to right-moving chiral lattices, and also Lorentzian lattices. In the latter case all the scalar products of vectors are understood with respect to the Lorentzian signature.

\section{APPENDIX B: CONSTRUCTION OF THE ALTERNATIVE $\mathrm{SO}(10)_{3}$}

Let us make a comment concerning the alternative $\mathrm{SO}(10)_{3}$ model presented in Sec. VII. There the construction, i.e., the set $\left\{V_{i}, k_{i j}\right\}$, satisfies the simplified rules, and the condition (3.24) follows. This same model may be obtained by a different set of $\left\{V_{i}, k_{i j}\right\}$ acting on the $N 1$ model. The vectors are, in addition to $V_{0}$,

$$
\begin{gathered}
V_{1}=\left(0\left(-\frac{1}{3} \frac{1}{3}\right)^{3}|| \frac{e_{1}}{3} 0\left(\frac{1}{3}\right) \mid\left(\frac{1}{3}\right)^{5} 0_{r}^{5}\left(\frac{2}{3}\right)_{r}\right), \\
V_{2}=\left(0(00)\left(-\frac{1}{2} \frac{1}{2}\right)^{2}|| \frac{e_{1}}{2}\left(\frac{1}{2}\right)^{2} \mid 0^{5} 0_{r}^{5} 0_{r}\right), \\
W_{1}=\left[0\left(0 \frac{1}{2}\right)^{3}|| 0^{2}\left(\frac{1}{2}\right) \mid\left(\frac{1}{2}\right)^{5} 0_{r}^{5} 0_{r}\right], \\
W_{2}=\left[0(00)\left(0 \frac{1}{2}\right)^{2}|| 0\left(\frac{1}{2}\right)^{2} \mid 0^{5} 0_{r}^{5} 0_{r}\right] .
\end{gathered}
$$

With the appropriate choice of $k_{i j}$, this gives the same model as the set $\left\{V_{i}, k_{i j}\right\}$ given in Sec. VII, with its massless spectrum shown in the second column in Table I.

The invariant sublattice $I\left(V_{1}\right)$ is the same in both cases, given by Eq. (5.9). However, the invariant sublattices for the other sectors are different in these two constructions. For the set $\left\{V_{i}, k_{i j}\right\}$ given in Sec. VII, $I\left(V_{1}+V_{2}\right)=I\left(V_{1}\right)$ and $I\left(V_{2}\right)=\Gamma^{4} \otimes \Gamma^{2,18}$, where $\Gamma^{2,18}$ is even self-dual. For the set $\left\{V_{i}, k_{i j}\right\}$ given here, $I\left(V_{1}+V_{2}\right)=\Gamma^{2} \otimes \Gamma^{6}$ and $I\left(V_{2}\right)=\Gamma^{2,2} \otimes \Gamma^{16}$, where $\Gamma^{16}$ corresponds to the $\mathrm{SO}(10)^{3} \otimes \mathrm{SO}(2)$ lattice, which is even but not self-dual. Now this set $\left\{V_{i}, k_{i j}\right\}$ does not satisfy the simplified rules, but it does satisfy the more general set of rules given in Sec. III. To check these points, it is convenient to have the values for $\chi(\overline{\alpha V}, \overline{\beta V}), f(\overline{\alpha V}, \overline{\beta V})$, and $\xi_{\overline{\beta V}}^{\overline{\alpha V}}$ in both cases. They are given in Table IV below. There, the first column corresponds to the $\left\{V_{i}, k_{i j}\right\}$ given in Sec. VII and the second column corresponds to the $\left\{V_{i}, k_{i j}\right\}$ given in this appendix. 
TABLE IV. The values $\chi(\overline{\alpha V}, \overline{\beta V}), f(\overline{\alpha V}, \overline{\beta V})$, and $\xi_{\overline{\beta V}}^{\overline{\alpha V}}$ for the original construction (first column) and the alternative construction (second column).

\begin{tabular}{|c|c|c|c|c|c|c|c|c|c|c|c|c|}
\hline \multirow[t]{2}{*}{$\overline{\alpha V} / \overline{\beta V}$} & \multicolumn{2}{|c|}{ 0 } & \multicolumn{2}{|c|}{$\overline{2 V_{1}+V_{2}}$} & \multicolumn{2}{|c|}{$V_{1}$} & \multicolumn{2}{|c|}{$V_{2}$} & \multicolumn{2}{|c|}{$\overline{2 V_{1}}$} & \multicolumn{2}{|c|}{$\overline{V_{1}+V_{2}}$} \\
\hline & 0 & 0 & 0 & 0 & 0 & 0 & 0 & 0 & 0 & 0 & 0 & 0 \\
\hline \multirow[t]{3}{*}{ 0 } & 0 & 0 & 0 & 0 & 0 & 0 & 0 & 0 & 0 & 0 & 0 & 0 \\
\hline & 1 & 1 & 1 & 1 & 1 & 1 & 1 & 1 & 1 & 1 & 1 & 1 \\
\hline & 0 & 0 & $-\frac{1}{4}$ & 0 & $\frac{1}{4}$ & 0 & 0 & 0 & $-\frac{1}{4}$ & 0 & $\frac{1}{4}$ & 0 \\
\hline \multirow[t]{3}{*}{$\overline{2 V_{1}+V_{2}}$} & 0 & 0 & 0 & 0 & 0 & 0 & $\frac{1}{2}$ & $\frac{1}{2}$ & 0 & 0 & 0 & 0 \\
\hline & 1 & 2 & 1 & 2 & 1 & 2 & 1 & 2 & 1 & 2 & 1 & 2 \\
\hline & 0 & 0 & $\frac{1}{4}$ & 0 & $-\frac{1}{4}$ & $-\frac{1}{4}$ & 0 & 0 & $\frac{1}{4}$ & $\frac{1}{4}$ & $-\frac{1}{4}$ & 0 \\
\hline \multirow[t]{3}{*}{$V_{1}$} & 0 & 0 & 0 & 0 & 0 & 0 & $\frac{1}{2}$ & $\frac{1}{2}$ & 0 & 0 & 0 & 0 \\
\hline & 3 & 3 & 1 & 1 & 3 & 3 & 1 & 1 & 3 & 3 & 1 & 1 \\
\hline & 0 & 0 & 0 & 0 & 0 & 0 & $\frac{1}{2}$ & 0 & 0 & 0 & 0 & 0 \\
\hline \multirow[t]{3}{*}{$V_{2}$} & 0 & 0 & $\frac{1}{2}$ & $\frac{1}{2}$ & $\frac{1}{2}$ & $\frac{1}{2}$ & 0 & 0 & $\frac{1}{2}$ & $\frac{1}{2}$ & $\frac{1}{2}$ & $\frac{1}{2}$ \\
\hline & 2 & 4 & -1 & -2 & -1 & -2 & 2 & 4 & -1 & -2 & -1 & -2 \\
\hline & 0 & 0 & $-\frac{1}{4}$ & 0 & $\frac{1}{4}$ & $\frac{1}{4}$ & 0 & 0 & $-\frac{1}{4}$ & $-\frac{1}{4}$ & $\frac{1}{4}$ & 0 \\
\hline \multirow[t]{3}{*}{$\overline{2 V_{1}}$} & 0 & 0 & 0 & 0 & 0 & 0 & $\frac{1}{2}$ & $\frac{1}{2}$ & 0 & 0 & 0 & 0 \\
\hline & 3 & 3 & 1 & 1 & 3 & 3 & 1 & 1 & 3 & 3 & 1 & 1 \\
\hline & 0 & 0 & $\frac{1}{4}$ & 0 & $-\frac{1}{4}$ & 0 & 0 & 0 & $\frac{1}{4}$ & 0 & $-\frac{1}{4}$ & 0 \\
\hline \multirow[t]{2}{*}{$\overline{V_{1}+V_{2}}$} & 0 & 0 & 0 & 0 & 0 & 0 & $\frac{1}{2}$ & $\frac{1}{2}$ & 0 & 0 & 0 & 0 \\
\hline & 1 & 2 & 1 & 2 & 1 & 2 & 1 & 2 & 1 & 2 & 1 & 2 \\
\hline
\end{tabular}

[1] L. J. Dixon, J. Harvey, C. Vafa, and E. Witten, Nucl. Phys. B261, 678 (1985); B274, 285 (1986).

[2] K. S. Narain, M. H. Sarmadi, and C. Vafa, Nucl. Phys. B288, 551 (1987).

[3] H. Kawai, D. C. Lewellen, and S.-H. H. Tye, Phys. Rev. Lett. 57, 1832 (1986); 58, 429(E) (1987); Nucl. Phys. B288, 1 (1987); I. Antoniadis, C. P. Bachas, and C. Kounnas, ibid. B289, 87 (1987).

[4] D. C. Lewellen, Nucl. Phys. B337, 61 (1990); J. A. Schwartz, Phys. Rev. D 42, 1777 (1990).

[5] For a partial list, see, e.g., L. E. Ibánez, J. E. Kim, H. P. Nilles, and F. Quevedo, Phys. Lett. B 191, 282 (1987); L. E. Ibánez, H. P. Nilles, and F. Quevedo, Nucl. Phys. B307, 109 (1988); A. Font, L. E. Ibánez, and F. Quevedo, ibid. B345, 389 (1990); I. Antoniadis, J. Ellis, J. Hagelin, and D. V. Nanopoulos, Phys. Lett. B 194, 231 (1987); 208, 209 (1988); 231, 65 (1989); I. Antoniadis, G. K. Leontaris, and J. Rizos, ibid. 245, 161 (1990); J. Lopez, D. V. Nanopoulos, and K. Yuan, Nucl. Phys. B399, 654 (1993); J. Lopez and D. V. Nanopoulos, ibid. B338, 73 (1989); Phys. Rev. Lett. 76, 1566 (1996); G. K. Leontaris, Phys. Lett. B 372, 212 (1996); D. Finnell, Phys. Rev. D 53, 5781 (1996); A. Maslikov, S. Sergeev, and G. Volkov, ibid. 50, 7440 (1994); A. Maslikov, I. Naumov, and G. Volkov, Int. J. Mod. Phys. A 11, 1117 (1996); D. Bailin, A. Love, and S.
Thomas, Phys. Lett. B 194, 385 (1987); B. R. Greene, K. H. Kirklin, P. J. Miron, and G. G. Ross, Nucl. Phys. B292, 606 (1987); R. Arnowitt and P. Nath, Phys. Rev. D 40, 191 (1989); A. Font, L. E. Ibánez, F. Quevedo, and A. Sierra, Nucl. Phys. B331, 421 (1990); A. E. Faraggi, Phys. Lett. B 278, 131 (1992); Phys. Rev. D 47, 5021 (1993); L. E. Ibánez, D. Lüst, and G. G. Ross, Phys. Lett. B 272, 251 (1991); S. Kachru, ibid. 349, 76 (1995); K. R. Dienes, Report No. IASSNS-HEP-95/97, hep-th/9602045, 1996 (unpublished); Report No. IASSNSHEP-96/64, hep-ph/9606467, 1996 (unpublished).

[6] See, e.g., S. Chaudhuri, S.-W. Chung, G. Hockney, and J. D. Lykken, Nucl. Phys. B456, 89 (1995); G. B. Cleaver, ibid. B456, 219 (1995); G. Aldazabal, A. Font, L. E. Ibánez, and A. M. Uranga, ibid. B452, 3 (1995).

[7] H. Kawai, D. C. Lewellen, J. A. Schwartz, and S.-H. H. Tye, Nucl. Phys. B299, 431 (1988).

[8] L. E. Ibánez, H. P. Nilles, and F. D. Quevedo, Phys. Lett. B 187, 25 (1987).

[9] Z. Kakushadze, G. Shiu, and S.-H. H. Tye, this issue, Phys. Rev. D 54, 7545 (1996).

[10] J. Erler, Nucl. Phys. B475, 597 (1996).

[11] Z. Kakushadze and S.-H. H. Tye, Phys. Rev. Lett. 77, 2612 (1996).

[12] Z. Kakushadze and S.-H. H. Tye, Report No. CLNS 95/1380, 
hep-th/9512156, 1995 (unpublished).

[13] K. S. Narain, Phys. Lett. 169B, 41 (1986); K. S. Narain, M. H. Sarmadi, and E. Witten, Nucl. Phys. B279, 369 (1986).

[14] C. Vafa, Nucl. Phys. B273, 592 (1986).

[15] K. R. Dienes and J. March-Russell, Nucl. Phys. B479, 113 (1996).
[16] S. Hamidi and C. Vafa, Nucl. Phys. B279, 465 (1987); L. J. Dixon, D. Friedan, E. Martinec, and S. Shenker, ibid. B282, 13 (1987); K. S. Narain, M. H. Sarmadi, and C. Vafa, ibid. B356, 163 (1991).

[17] A. H. Chamseddine, J.-P. Derendinger, and M. Quirós, Nucl. Phys. B326, 497 (1989). 